\title{
MUON-NEUTRINO ELECTRON ELASTIC SCATTERING AND A SEARCH FOR THE MUON-NEUTRINO MAGNETIC MOMENT IN THE NOVA NEAR DETECTOR
}

Approved by:

Dr. Thomas E. Coan

Professor of Physics

Dr. Ryszard Stroynowski

Professor of Physics

Dr. Roberto Vega

Professor of Physics

Dr. Xinlei Wang

Professor of Statistics 
MUON-NEUTRINO ELECTRON ELASTIC SCATTERING

\section{AND A SEARCH FOR THE MUON-NEUTRINO MAGNETIC MOMENT IN THE NOVA NEAR DETECTOR}

A Dissertation Presented to the Graduate Faculty of the

Dedman College

Southern Methodist University

in

Partial Fulfillment of the Requirements

for the degree of

Doctor of Philosophy

with a

Major in Physics

by

Biao Wang

B.S., Lanzhou University, 2009

December 16, 2017 


\section{ACKNOWLEDGMENTS}

It has been such a fortunate that I can study physics in Southern Methodist University, one of the most beautiful campuses in the North America with the intense amount of research support that has been provided for each student. Many people have helped me in this process from the Physics department, such as Jun Gao, Tiankuan Liu, Florian Lyonnet and other graduate students.

I would like to express my sincere gratitude to Professor Thomas E. Coan, my advisor and admired mentor, for his significant help in my Ph.D. career. It is what Dr. Coan did makes me understand how the United States has become the most powerful country over the past two centuries. Each time we discuss the physics in Toms office, his insights on the experimental HEP concept and experience of solving problems impress me deeply.

Outside the research discussion, Dr. Coan's philosophies have grown in my mind and thus shaped my personality in many aspects. Those theories helped me choose priorities between multi-tasks, get my visions broaden, keep organized in every precious strategic resource. And at the same time, he gave me lots of mental supports that help me mature up and become patient, calm and steady to focus on my project.

Dr. Coan is also a very versatile expert that can help graduate students built up other skill trees. He is very good at electronic hardware design, photographing and high-performance computing. In short, I wish every graduate student had an advisor like Dr. Coan to help them launch towards the right career.

I thank Dr. Roberto Vega and Dr. Kent Hornbostel for their modern physics lectures. Taking their quantum courses has been one of the most enjoyable experiences on the SMU campus, since the lectures are so clear and evolutionary in sequence that the beginners can attain whole new level of understanding in the theoretical approach. I specially thank Prof. Vega for help me developing the motivation and insights of the theoretical background in 
this thesis. Also, his comments inspired me several new ideas about the source of ultra high energy neutrino flux.

It has also been a great honor for me to have the chance working with Dr. Ryszard Stroynowski. What has been most inspiring is his guidance and insights in many broad areas of high energy physics as well as his sharper thinking and mental arithmetic, which are much better than lots of young scientists. To meet his requirement, I had to push myself day and night. From scientific writing to research analysis and life wisdom, I have learned a lot from him.

I thank Dr. Xinlei Wang from the Statistics department at SMU. Her valuable discussions about alternative methods in deep learning algorithms will help me in future analysis. I also noticed that some of her research might also benefit my accelerator-based lightweight dark matter searches and proton decay searches in the DUNE experiment.

I look forward to working with them in the future. There are also a lot of people from the NOvA collaboration that had helped most, such as Dr. Kirk Bays, Dr. Brian Rebel, Dr. Gavin Davies and Dr. Chris Backhouse. Besides the collaborators that I have been working closely with in both NOvA and DUNE collaborations, I want to thank the following scientists for their great assistance.

I want to express my gratitude to Dr. Alec Habig, Dr. Craig Dukes, Dr. Jonathan Paley and Dr. Matthew Strait, who were giving me very useful guidance throughout numerous consultations, from detailed modifications to long-run plans. I especially thank the production group and computing division at Fermilab. I thank Dr. Nathan Mayer, Dr. Robert Hatcher and Dr. Libo Jiang for the GENIE support. I also thank Dr. Jarek Nowak, Dr. Jianming Bian and Dr. Alexander Radovic for participating in this work in its early stage.

I have to thank Dr. Ryan Patterson and Dr. Daniel Cronin-Hennessy who had sincerely helped the analysis of neutrino-electron elastic scattering. Their suggestions and comments addressed the most controversial points in this analysis.

I thank Dr. K.S. Babu for inspiring me the motivation and details about the neutrino transitional magnetic moments at SLAC summer school in 2015. I appreciate Dr. de Gou- 
vea, Dr. Carlo Giunti, and Dr. Yufeng Li for theoretical insights and Dr. Xin Qian for experimental suggestions during Neutrino 2016.

I thank our spokesperson, Dr. Mark Messier, who taught me three important pieces of advice during DPF 2015: (1) Be a well-mannered person so that other collaborators can rely on you. (2) After you succeed in certain skills, try to help others as much as you can. (3) When you are underestimated by others, never complain or get depressed, but do it better next time.

I thank Dr. Abigail Woolley for academic writing counseling. I now have my own advice for new graduate students. Even if you are as much of a genius as Leonardo da Vinci, the first lesson always starts with somethings simple as the painting of eggs, thousands of eggs. 
Muon-Neutrino Electron Elastic Scattering

and a Search for the Muon-Neutrino Magnetic Moment

in the NOvA Near Detector

Advisor: Dr. Thomas E. Coan

Doctor of Philosophy degree conferred December 16, 2017

Dissertation completed December 11, 2017

We use the NOvA near detector and the NuMI beam at Fermilab to study the neutrinoelectron elastic scattering and the muon neutrino magnetic process beyond the Standard Model physics. The particle identifications of neutrino on electron elastic scattering are trained by using the multi-layer neural networks. This thesis provides a general discussion of this technique and shows a good agreement between data and $\mathrm{MC}$ for the neutrino-electron elastic weak scattering. So that benefiting from the precise cross-section of this channel, we are able to tune the neutrino beam flux simulation in the future. Giving the exposure of $3.62 \times 10^{20}$ POT in the NOvA near detector, we report $1.58 \times 10^{-9} \mu_{B}$ as an upper limit on the muon neutrino magnetic moment at $90 \%$ conference level. We also find a sensitivity of $8 \times 10^{-10} \mu_{B}$ at $90 \%$ conference level for three years NuMI designed intensity under the assumption that the standard model background is well known. 


\section{TABLE OF CONTENTS}

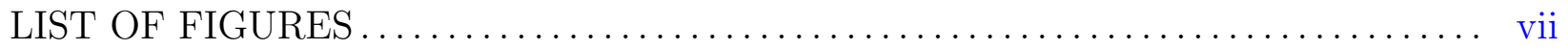

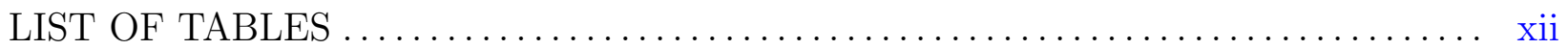

\section{CHAPTER}

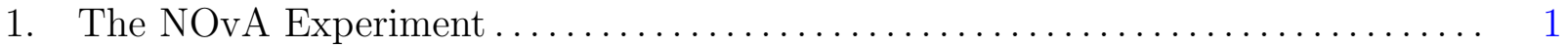

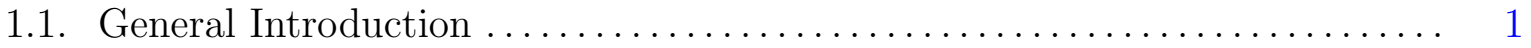

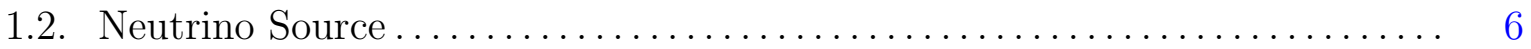

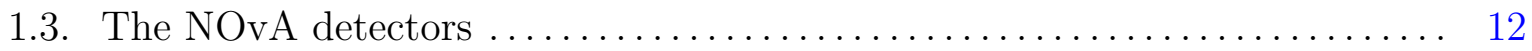

1.4. Data Acquisition .................................. 15

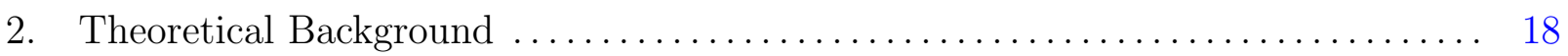

2.1. Neutrino Electron Elastic Scattering ....................... 19

2.2. Muon Neutrino Magnetic Moment ............................. 22

2.2.1. Magnetic Moment for Dirac Neutrinos ....................... 22

2.2.2. CPT Properties of EM Field for Majorana Neutrinos.............. 23

2.2.3. Hypothesis of Large Transition Magnetic Moments for Majorana

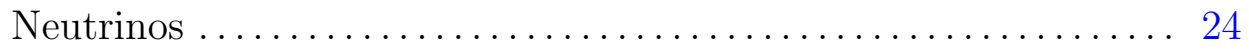

2.3. The Impacts of NMM in Cosmology and Astrophysics ............... 29

3. Event Reconstruction and Classifier ........................... 33

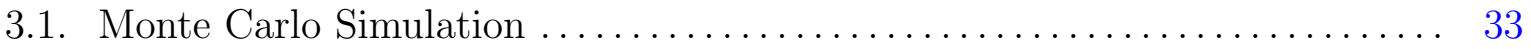

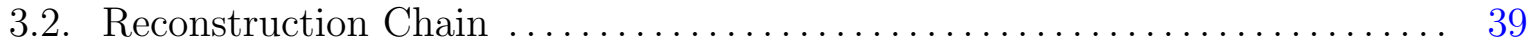

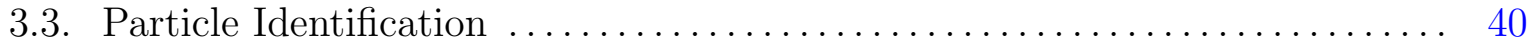

3.3.1. Log Likelihood Identification $\ldots \ldots \ldots \ldots \ldots \ldots \ldots \ldots \ldots \ldots \ldots \ldots \ldots \ldots$

3.3.2. Neutral Current Pions as Major Background ................ 53

3.4. Rough Sensitivity for Neutrino Electron Elastic Scattering .......... 60

4. Neutrino-Electron Elastic Weak Scattering ...................... 64 
4.1. Strategy for High-Energy Flux Constraint...................... 64

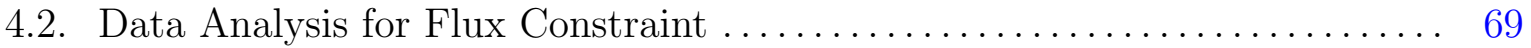

4.3. Systematic Uncertainties of Beam Simulation $\ldots \ldots \ldots \ldots \ldots \ldots \ldots \ldots \ldots$

5. Analysis of Muon Neutrino Magnetic Moment . . . . . . . . . . . . . . . . . . . 79

5.1. Systematic Uncertainties and Selection Optimization . . . . . . . . . . 79

5.2. Statistical Methods for the Upper Limit $\ldots \ldots \ldots \ldots \ldots \ldots \ldots \ldots \ldots \ldots$

5.2.1. Chi-Squared Quantile Method ....................... 90

5.2.2. Feldman-Cousins Method $\ldots \ldots \ldots \ldots \ldots \ldots \ldots \ldots \ldots \ldots \ldots \ldots \ldots \ldots \ldots \ldots \ldots \ldots$

5.2.3. The Profile Likelihood Method ....................... 95

5.2.4. Preliminary Result for NOvA $2016 \ldots \ldots \ldots \ldots \ldots \ldots \ldots \ldots \ldots$

5.3. Future Sensitivity for the Full Dataset $\ldots \ldots \ldots \ldots \ldots \ldots \ldots \ldots \ldots \ldots \ldots$

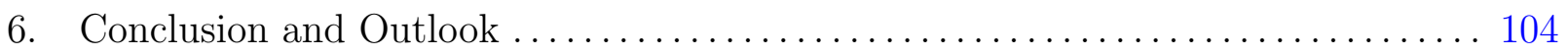

\section{APPENDIX}

BIBLIOGRAPHY 


\section{LIST OF FIGURES}

Figure

Page

1.1 Regions of $\delta_{C P}$ vs. $\sin ^{2} \theta_{23}$ parameter space. $\ldots \ldots \ldots \ldots \ldots \ldots \ldots \ldots \ldots \ldots$

1.2 Neutrino-nucleus neutral current scattering disappearance for sterile neu-

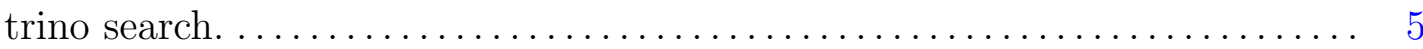

1.3 The cartoon structure of NuMI beam, the diagram is originally from Ref. [4]. . 6

1.4 Off-axis muon neutrino from pion decay. $\ldots \ldots \ldots \ldots \ldots \ldots \ldots \ldots \ldots \ldots \ldots$

1.5 Muon neutrino energy vs its parent pion energy, $[46] . \ldots \ldots \ldots \ldots \ldots \ldots \ldots$

1.6 Off-axis angles comparison at far detector $\ldots \ldots \ldots \ldots \ldots \ldots \ldots \ldots \ldots \ldots$

1.7 Locations of NOvA Near Detector and Far Detector. The NOvA near detector seats underground at Fermilab while the far detector is constructed on the surface at Ash River, MN. .......................... 10

1.8 Muon neutrino energy spectrum at near detector [46] and the components

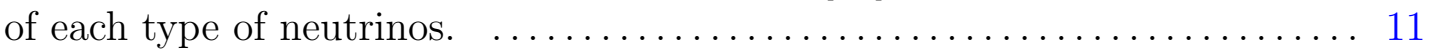

1.9 Structure of NOvA detectors. The scintillation light is generated by charged particles and collected by a looped wavelength-shifting fiber in each cell. Each avalache-photodiode (APD) reads out 32 cells and connects to a front-end-board (FEB). The FEB digitizes the readout from APD and sends the signal to a data-concentrator-module (DCM) $[105,107]$. . ...... 12

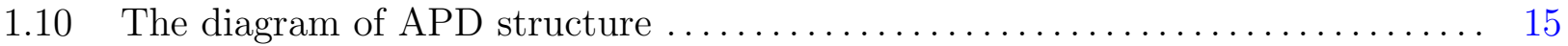

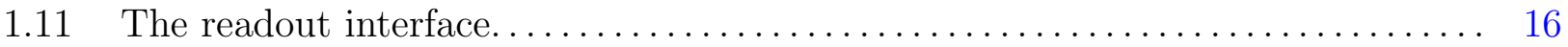

1.12 The logic structure of DAQ system, diagram is originally from Ref. [23,54]... 17

2.1 The Feynman diagrams for $\nu$-e elastic scatterings. $\ldots \ldots \ldots \ldots \ldots \ldots \ldots \ldots \ldots$

2.2 Magnetic scattering due to transitional magnetic moments. The CPT invariance force a Majorana neutrino of a given spin to rotate into one of

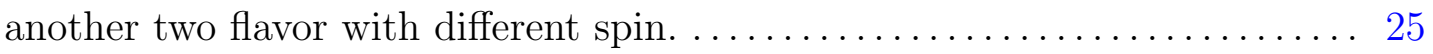

2.3 The differential cross-section for $\nu_{\mu}$-e scattering for various values of neutrino magnetic moment by using Eq. 2.3 and Eq. 2.17. ................ 26 
2.4 Triangle relations for Majorana neutrino magnetic moments. Plot is taken

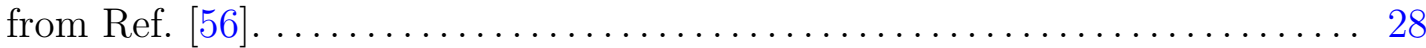

2.5 Contours of constant $N_{\text {eff }}$ in the $\mu_{\mu \tau}$ versus $\mu_{e \mu}$ plane. The solid contours correspond to $\mu_{e \tau}=10^{-10} \mu_{B}$ and the dashed contours correspond to $\mu_{e \tau}=4.9 \times 10^{-10} \mu_{B}$. This plot is taken from Ref. [95] .............. 30

2.6 The change in primordial relative abundances of ${ }^{4} \mathrm{He}, \mathrm{D},{ }^{3} \mathrm{He}$, and ${ }^{7} \mathrm{Li}$ as a function of the transition neutrino magnetic moment $\mu_{e \mu}$. The solid lines show the results when $\mu_{e \tau}=10^{-11} \mu_{B}$ (black - $\mu_{\mu \tau}=10^{-11} \mu_{B}$, red - $\mu_{\mu \tau}=4 \times 10^{-10} \mu_{B}$, and blue $\left.-\mu_{\mu \tau}=6 \times 10^{-10} \mu_{B}\right)$. The dashed lines are the results for $\mu_{e \tau}=6 \times 10^{-10} \mu_{B}$ with the colors representing the same values of $\mu_{\mu \tau}$ that they did in the solid case. The plot and caption

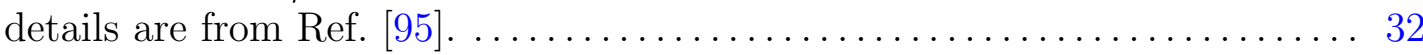

3.1 The tracks of mesons in the magnetic horns, $[4] \ldots \ldots \ldots \ldots \ldots \ldots \ldots \ldots \ldots \ldots$

3.2 Event display for a $2 \mathrm{GeV}$ electron shower from a $\nu_{\mu}$-e elastic scattering in

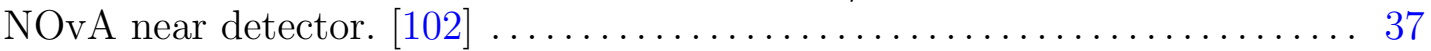

3.3 Reconstruction Chain for a general event in the NOvA experiment, the plot

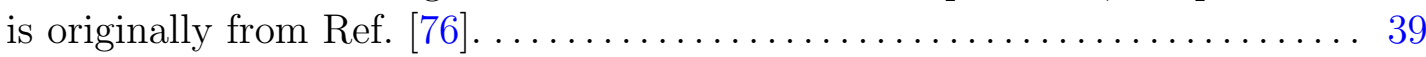

3.4 The event displays for $\nu_{\mu}-N$ charge current scattering, $\nu_{e}-N$ charge current scattering and $\nu$ - $N$ neutral current scattering. .................... 41

3.5 The diagram shows the strategy of this analysis, the $\nu_{\mu}-N$ charge current scattering, $\nu_{e}-N$ charge current scattering and $\nu-N$ neutral current scattering are the background to be removed........................ 42

3.6 The structure of the NOvA detectors with vertical and horizontal alternating orientations, this diagram is originally from Ref. $[21] \ldots \ldots \ldots \ldots \ldots \ldots . \ldots 44$

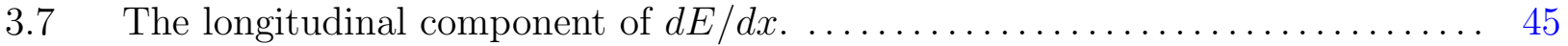

3.8 Longitudinal $d E / d x$ for electrons (red) and muons (blue)............... 45

$3.9 \quad$ The transverse cell definition for $d E / d x \ldots \ldots \ldots \ldots \ldots \ldots \ldots \ldots \ldots \ldots \ldots \ldots \ldots \ldots \ldots$

3.10 Comparing log likelihood of electron to $\gamma$, similar methods as described in

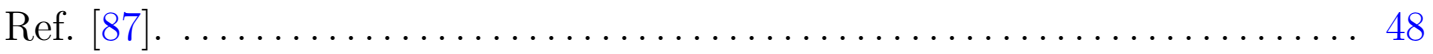

3.11 Comparing log likelihood of electron to neutral pion, similar methods as

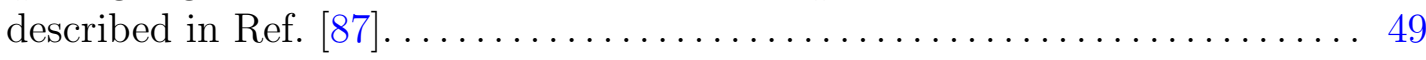

3.12 Comparing log likelihood of electron to muon, similar methods as described in Ref. [87]........................................ 49 
3.13 Comparing log likelihood of electron to charged pions, similar methods as

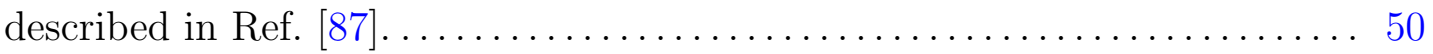

3.14 Comparing log likelihood of electron to proton, similar methods as described

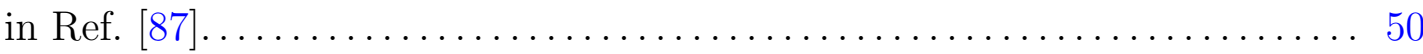

3.15 Comparing log likelihood of electron to neutron, similar methods as described in Ref. [87]..................................... 51

3.16 Performance for ES-CC PID. This is a plot after pre-selections. The performance of this PID is originally quite polarized, most of the $\nu_{\mu}$ charge current events were taged with ES-CC PID $<0.3$, so that they were removed in pre-selections. ................................ 52

3.17 A typical example of $\nu_{\mu}$ on electron elastic scattering resulted electromagnetic shower. . . . . . . . . . . . . . . . . . . . . . . . . . . . . . 53

3.18 An example of pion induced electromagnetic shower, the shower property is very similar as the scattered electron shower. ..................... 54

3.19 Another example of neutral current $\pi^{0}$ background, the shower property is also close to the scattered electron shower. ....................... 55

3.20 Energy deposition in CHARM detector.(a) electrons, (b) neutral pions, [50]... 56

3.21 The $\mathrm{dE} / \mathrm{dx}$ distribution in the first plane......................... 56

3.22 The $\mathrm{dE} / \mathrm{dx}$ distribution in the second plane. ....................... 57

3.23 The $\mathrm{dE} / \mathrm{dx}$ distribution in the third plane......................... 57

3.24 The $\mathrm{dE} / \mathrm{dx}$ distribution in the fourth plane......................... 58

3.25 Performance for ES-NC $\pi^{0}$ PID. Plot is made after pre-selections and ES-CC

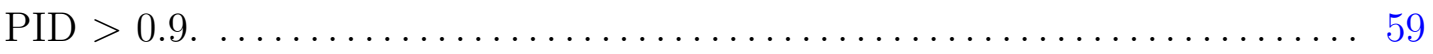

3.26 Tuning $T \theta^{2}$ cut for $T>1 \mathrm{GeV}$. See more discussions in Chapter four. ...... 61

3.27 Tuning cut $\mathrm{T}$ for higher edge of the first analysis NMM signal region. $\quad \ldots \ldots \ldots 62$

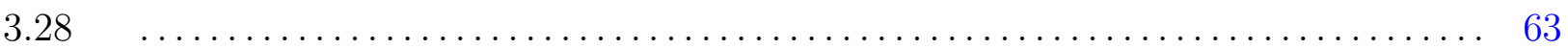

4.1 ND muon neutrino reconstructed energy distributions, data and MC with full systematics band, for neutrino energy, POT normalized. ............ 65

4.2 A typical charge current deep inelastic scattering in NOvA near detector. Note that the leading hadronic shower appearance is about 12 meters away from the interaction vertex............................. 66 
4.3 A scattered electron $(28.5 \mathrm{GeV})$ produced by a high energy muon neutrino $(29.7 \mathrm{GeV})$ interaction in NOvA near detector................. 67

4.4 The diagram for lightweight dark matter (LDM) produced in the proton on target collision and scatter through a mediator in the NOvA ND. ........ 68

4.5 ND muon neutrino electron elastic scattering (ES) reconstructed $T \theta^{2}$ distributions, the optimal cut is chosen at $T \theta^{2}<0.003 \mathrm{GeV} \times \operatorname{Radian}^{2}$. ...... 69

4.6 ND muon neutrino electron elastic scattering reconstructed $\mathrm{T}(\mathrm{GeV})$ distributions, after $T \theta^{2}<0.003 \mathrm{GeV} \times$ Radian $^{2}$ cut shown in Fig. 4.5........ 71

4.7 ND muon neutrino electron elastic scattering reconstructed $\mathrm{T}(\mathrm{GeV})$ distributions, after $T \theta^{2}<0.003 \mathrm{GeV} \times$ Radian $^{2}$ cut with $100 \mathrm{~cm}<\mathrm{Z}<$ $1200 \mathrm{~cm}$ fiducial volume.................................. 73

4.8 ND muon neutrino spectrum with ancestors......................... 74

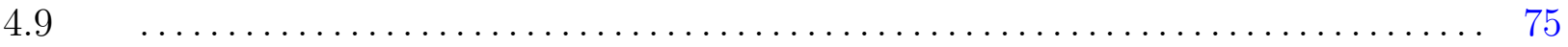

4.10 Diagram of the neutrino beam facility in FHC mode.................... 76

4.11 What the proton beam sees as it travels through the NuMI baffle........... 77

5.1 Monte Carlo components for the $1.8 \times 10^{21}$ POT dataset. In this plot, all the $\mathrm{MC}$ components are stacked together, so that the area under the red solid line indicates the Standard Model prediction while the area under the blue solid line represents the sum of $\nu_{\mu}$-e magnetic scattering and SM background. The rest of the plots in this chapter follow the

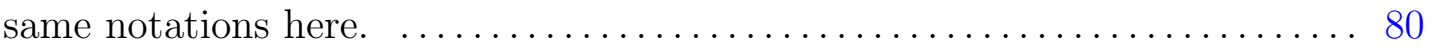

$5.2 \quad$ Syst. shift caused by $T \theta^{2}$ cut. $\ldots \ldots \ldots \ldots \ldots \ldots \ldots \ldots \ldots \ldots \ldots \ldots \ldots \ldots \ldots \ldots$

$5.3 \quad$ Syst. shift caused by scintillation modeling. ........................ 83

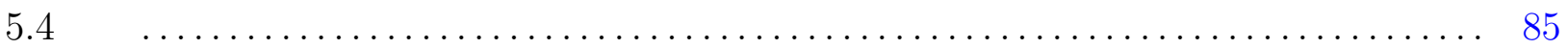

5.5 Data/MC comparison for searching for neutrino magnetic moment by using $3.62 \times 10^{20} \mathrm{POT}$ in NOvA near detector. In the signal selection region, we see 25 events in data while 23.78 events in the MC SM prediction. .... 87

$5.6 \quad$ Pseudocode for the Feldman-Cousins method. . ....................... 92

$5.7 \quad$ Feldman-Cousins for $68.3 \%$ C.L.............................. 93

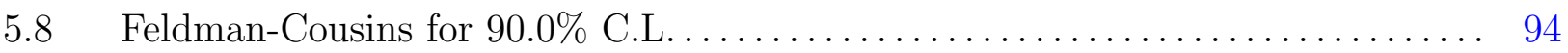

5.9 Using the profile likelihood ratio function $\lambda$ to estimate an upper limit of $s$ at $68.3 \%$ C.L., with $b=23.78$ and $N=25 \ldots \ldots \ldots \ldots \ldots \ldots \ldots \ldots \ldots$ 
5.10 Using the profile likelihood ratio function $\lambda$ to estimate an upper limit of $s$ at $90.0 \%$ C.L., with $b=23.78$ and $N=25 \ldots \ldots \ldots \ldots \ldots \ldots \ldots \ldots \ldots \ldots$

5.11 The simulated $T$ distribution for $1.8 \times 10^{21}$ POT. For a value of $\mu_{\nu}=10^{-9} \mu_{B}$, we see 21 simulated EM ES events over the Standard Model prediction of 131 events.............................................. 100

5.12 The future sensitivity for muon neutrino magnetic moment of $10^{-9} \mu_{B}$ by using $1.8 \times 10^{21}$ POT. The left (black) vertical line is the mock data and the right (red) vertical line is the $s\left(10^{-9} \mu_{B}\right)+b$. The mock data is the same amount of events number as Standard Model prediction. The confidence level for constraining the size of $\mu_{\nu}=10^{-9} \mu_{B}$ for a fixed number of observed events 131, is calculated by using the area on the right hand side of the black line divided by the total area in the distribution.

5.13 The future sensitivity for muon neutrino magnetic moment at $90 \%$ C.L. by using $1.8 \times 10^{21}$ POT. The mock data has the same number of events as the Standard Model predicted. We find $s_{u p}=1.28 \sqrt{b}=14.6$ events, corresponding to $\mu_{\nu}=8 \times 10^{-10} \mu_{B}$ 


\section{LIST OF TABLES}

Table

Page

1.1 Size Comparison of Far Detector and Near Detector . . . . . . . . . . . . 13

$2.1 \quad \mathrm{~W}$ and $\mathrm{Z}$ exchange in t-channel and s-channel. . . . . . . . . . . . . . . 19

2.2 The constants of coupling constants in different $\nu$-e scatterings, where $g_{A}=$ $-\frac{1}{2}, g_{V}=-\frac{1}{2}+2 \sin ^{2} \theta_{w} \quad[59] \ldots \ldots \ldots \ldots \ldots \ldots \ldots \ldots \ldots \ldots \ldots \ldots \ldots \ldots \ldots \ldots \ldots \ldots \ldots \ldots \ldots \ldots \ldots$

2.3 The C, P, T properties for electromagnetic field. . . . . . . . . . . . . . . 24

2.4 List of previous experiments that provided an upper limit of muon neutrino

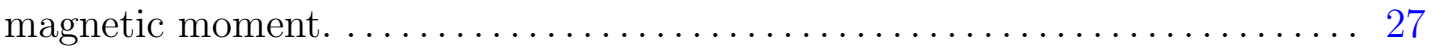

3.1 The material list in the NOvA near detector GDML files. . . . . . . . . . 35

3.2 The isotope list in the NOvA near detector GDML files. . . . . . . . . . . . 36

3.3 Comparing significance for different $\mathrm{T} \theta^{2}$ cuts. Corresponding to Fig. $3.26 \ldots \ldots \quad 60$

3.4 Tuning cut T for higher edge of NMM signal region. Corresponding to Fig. 3.27. 62

4.1 Comparing the data to prediction for $1 \mathrm{GeV}<\mathrm{T}<3 \mathrm{GeV} \ldots \ldots \ldots \ldots \ldots .71$

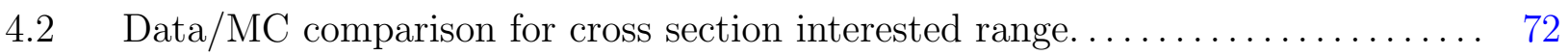

4.3 The data/MC for $T>1 \mathrm{GeV}$ and $100 \mathrm{~cm}<\mathrm{Z}<1200 \mathrm{~cm} . \ldots \ldots \ldots \ldots \ldots . . \ldots 2$

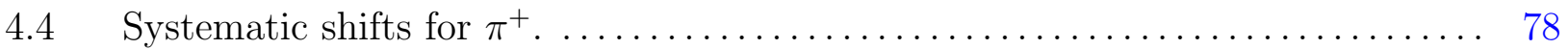

5.1 Summary of the systematic uncertainties for number of selected events. The first column lists the source of each systematic shift, the second column lists the change of number of selected events for $\nu_{\mu}-e$ magnetic scattering with $\mu_{\nu_{\mu}}=10^{-9} \mu_{B}$ and the third column lists the change in the Standard Model background............................. 86

$5.2 \quad$ The table for $\frac{1}{2} \Delta \chi_{0}^{2}$ values. $\ldots \ldots \ldots \ldots \ldots \ldots \ldots \ldots \ldots \ldots \ldots \ldots \ldots \ldots \ldots$

5.3 A comparison of the preliminary results from different statistical methods..... 99 
This dissertation is dedicated to my family. 


\section{CHAPTER 1}

The NOvA Experiment

\subsection{General Introduction}

There are three types of active neutrinos in nature so far as we know [45,79]. Bruno Pontecorvo made the first suggestion of neutrino oscillations in 1957, by which a transformation of electron neutrino to one of other types was postulated in order to solve the solar neutrino puzzle [62]. After the muon neutrino had been discovered in 1962, Maki, Nakagawa, and Sakata discussed the possibility of muon neutrino to electron neutrino oscillations $[52,79]$.

For this reason, the neutrino mixing matrix is called the PMNS matrix [52]. This unitary rotation matrix gives the relationship between the neutrino weak eigenstates and the neutrino mass eigenstates.

$$
\left(\begin{array}{c}
\nu_{e} \\
\nu_{\mu} \\
\nu_{\tau}
\end{array}\right)=U_{P M N S} \times\left(\begin{array}{c}
\nu_{1} \\
\nu_{2} \\
\nu_{3}
\end{array}\right)
$$

As a analogue of CKM quark mixing matrix, we consider that there are superpositions between the orthogonal state masses and flavor states of 3 types of neutrinos. Each flavor state is a linear combination of mass states with coefficients and phases, so that the $\nu_{\mu}$ to $\nu_{e}$ oscillation can be expressed as in Eq. 1.2

$$
P\left(\nu_{\mu} \rightarrow \nu_{e}\right)=\left|\left\langle\nu_{\mu}(t) \mid \nu_{e}(0)\right\rangle\right|^{2}=\left|\sum_{i=1}^{3}\left(\left\langle\nu_{\mu} \mid \nu_{i}\right\rangle e^{-i E t / \hbar}\left\langle\nu_{i} \mid \nu_{e}\right\rangle\right)\right|^{2}
$$

Here, $\hbar$ is the reduced Planck constant and $t$ represents the time between observation of the initial and final states. Since the oscillation is time dependent, for neutrinos moving with a 
speed of light, it is convenient to translate time into distance $L=c t$.

We use a 3 by 3 matrix to represent the elements of the superpositions in the PMNS matrix.

$U_{P M N S}=\left(\begin{array}{ccc}1 & 0 & 0 \\ 0 & \cos \theta_{23} & \sin \theta_{23} \\ 0 & -\sin \theta_{23} & \cos \theta_{23}\end{array}\right)\left(\begin{array}{ccc}\cos \theta_{13} & 0 & \sin \theta_{13} e^{-i \delta_{C P}} \\ 0 & 1 & 0 \\ -\sin \theta_{13} e^{-i \delta_{C P}} & 0 & \cos \theta_{13}\end{array}\right)\left(\begin{array}{ccc}\cos \theta_{12} & \sin \theta_{12} & 0 \\ -\sin \theta_{12} & \cos \theta_{12} & 0 \\ 0 & 0 & 1\end{array}\right)$

Literally, from neutrino to anti-neutrino it is the C-transformation, and from left-handed neutrino to right-handed neutrino it is the parity inversion. And since within the Standard Model (SM) there are only left-handed neutrino and right-handed anti-neutrino, we can measure the $\mathrm{CP}$ violation phase $\delta_{C P}$ in the neutrino sector. If this $\delta_{C P}$ value locates outside the range between zero and $\pi$, we confirm that $\mathrm{CP}$ is violated. This $\mathrm{CP}$ violation may provide strong support for leptogenesis mechanism for generating the matter-antimatter asymmetry in the universe [93].

Therefore, we summarize that the neutrino oscillations are functions of probabilities in terms of mass eigenstates difference, $\mathrm{CP}$ violation phase $\delta_{C P}$ and mixing angles. For the muon-neutrino beam, we have approximate formula

$$
\operatorname{Prob}\left(\nu_{\mu} \rightarrow \nu_{e}\right) \approx \sin ^{2} 2 \theta_{13} \sin ^{2} \Delta_{32}\left(\sin ^{2} \theta_{23}-\sin \delta_{C P} \sin \Delta_{21} \frac{\sin 2 \theta_{12} \sin 2 \theta_{23}}{2 \sin \theta_{13}}\right)
$$

for electron-neutrino appearance in vacuum, where $\Delta_{i j} \equiv \frac{\Delta m_{i j}^{2} L}{4 E}$. And

$$
\operatorname{Prob}\left(\nu_{\mu} \rightarrow \nu_{\mu}\right) \approx 1-\sin ^{2} 2 \theta_{23} \sin ^{2} \Delta_{32}
$$

for muon-neutrino disappearance in vacuum. 
Similarly, for anti-muon-neutrino beam, we have

$$
\operatorname{Prob}\left(\bar{\nu}_{\mu} \rightarrow \bar{\nu}_{e}\right) \approx \sin ^{2} 2 \theta_{13} \sin ^{2} \Delta_{32}\left(\sin ^{2} \theta_{23}+\sin \delta_{C P} \sin \Delta_{21} \frac{\sin 2 \theta_{12} \sin 2 \theta_{23}}{2 \sin \theta_{13}}\right)
$$

for anti-electron-neutrino appearance with a sign flip in the $\delta_{C P}$ term. Also,

$$
\operatorname{Prob}\left(\bar{\nu}_{\mu} \rightarrow \bar{\nu}_{\mu}\right)=\operatorname{Prob}\left(\nu_{\mu} \rightarrow \nu_{\mu}\right)
$$

by assuming the CPT is invariable in vacuum. 


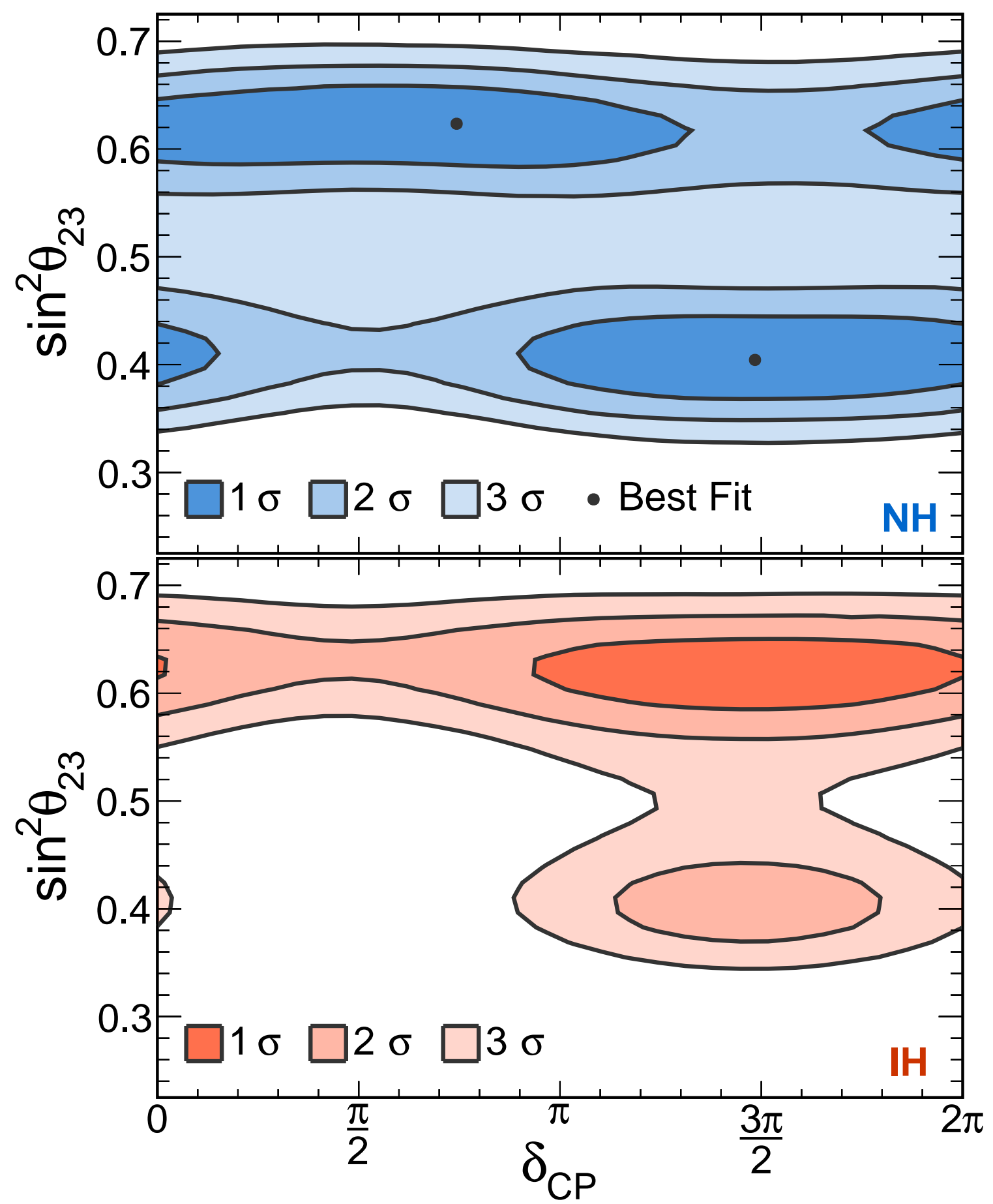

Figure 1.1. Regions of $\delta_{C P}$ vs. $\sin ^{2} \theta_{23}$ parameter space, results from NOvA second analysis [1]. The top panel corresponds to normal mass hierarchy and the bottom panel to inverted hierarchy. The hypothesis of inverted mass hierarchy in the lower octant is disfavored at greater than $93 \%$ C.L. for the whole range of $\delta_{C P}$ values. 
The NOvA experiment goals are to measure the main parameters of neutrino oscillations including their mass hierarchy [81], CP phase with octant of the squared sine of mixing angle $\theta_{23}$ as shown in Fig. 1.1. Also, to provide an estimate of the neutrino-nucleus neutral current scattering disappearance for sterile neutrino search [3] as the oscillation involved different mass states of sterile neutrinos is shown in Fig. 1.2.

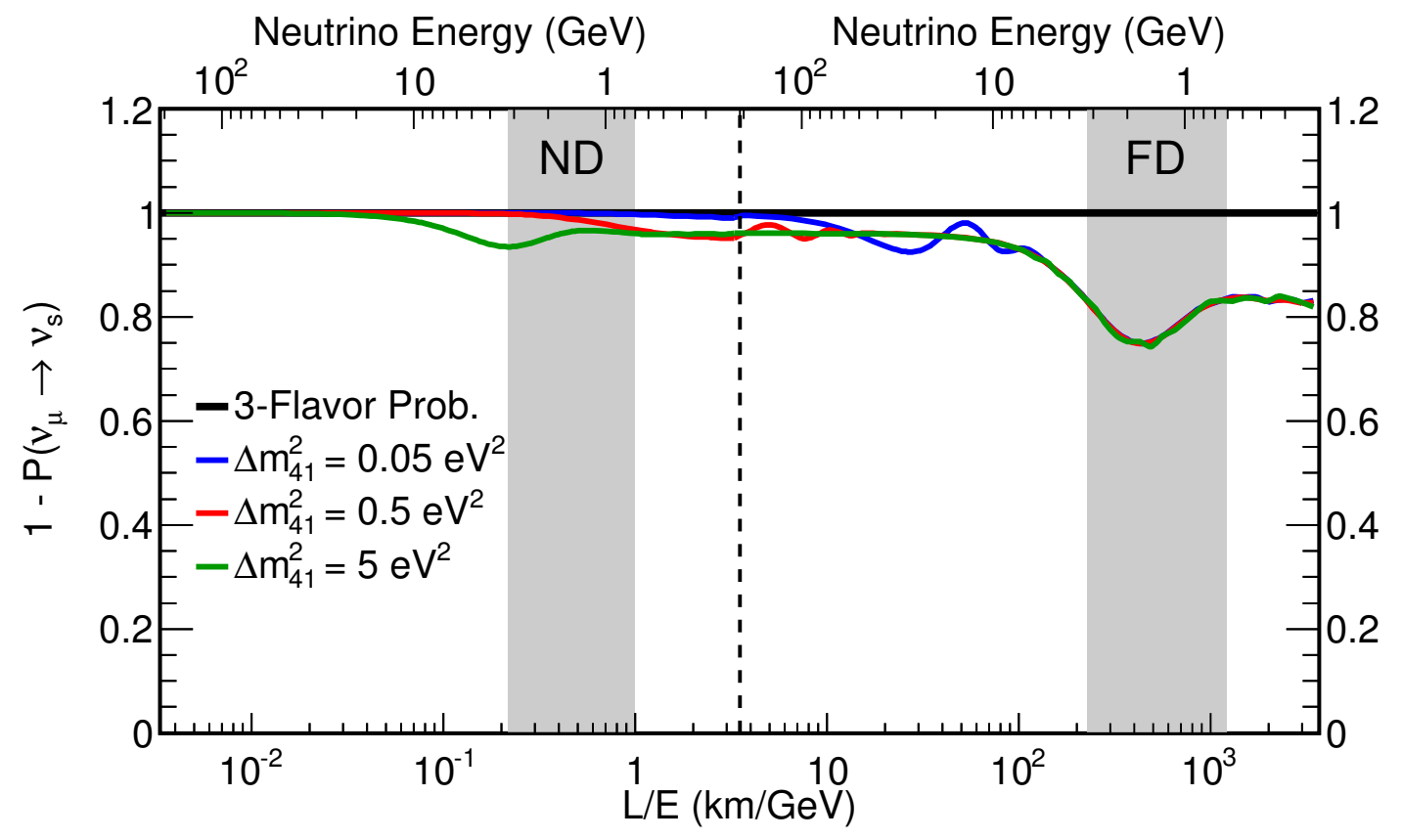

Figure 1.2. Neutrino-nucleus neutral current scattering disappearance for sterile neutrino search.

The benefits of a large far detector allow us to study astroparticle topics such as searches for supernovas, magnetic monopoles, east-west effects and others. Since the near detector is close to the NuMI target, there will be sufficient neutrino flux intensity to accumulate significant statistics for studying of other topics: deep inelastic scattering, quasi-elastic interactions, kaon decays and $\nu$-e elastic scattering with its applications beyond the Standard Model physics.

In this thesis, after providing a brief introduction and overview of hardware system in the NOvA experiment, I focus on the neutrino-electron elastic scattering and muon neutrino 
magnetic moment.

\subsection{Neutrino Source}

NOvA experiment is using the NuMI muon-neutrino beams from Fermilab, aiming at the far detector located at Ash River in Minnesota with a 14.6 mrad off-axis angle, so that the energy spectrum of $\nu_{\mu}$ is peaked around $2 \mathrm{GeV}$ [81].

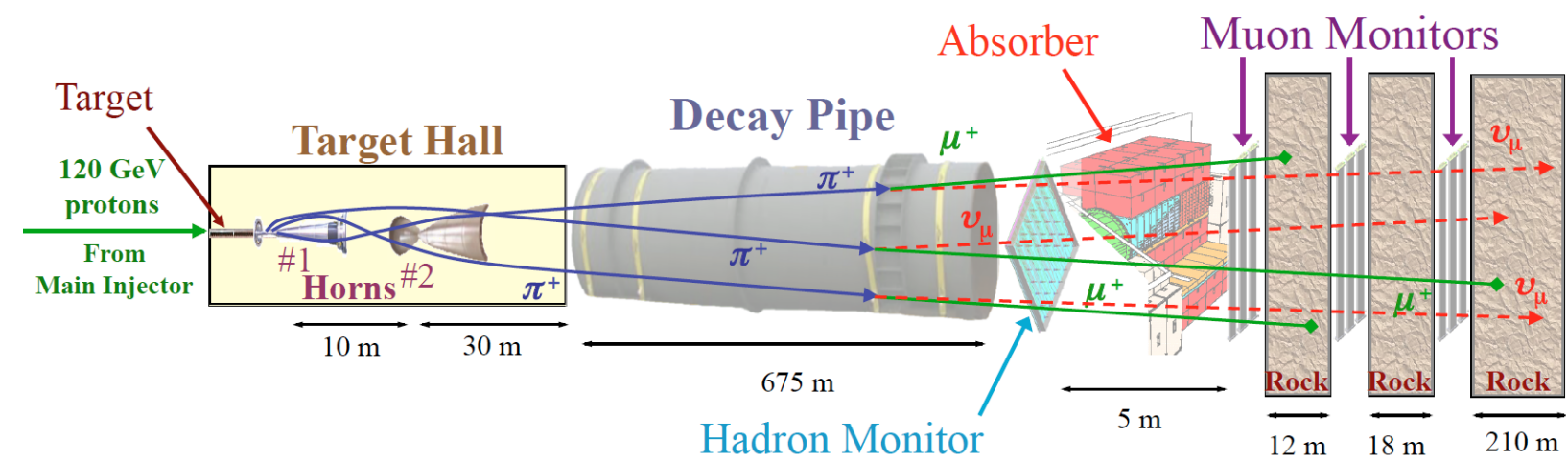

Figure 1.3. The cartoon structure of NuMI beam, the diagram is originally from Ref. [4].

Neutrinos at the Main Injector (NuMI) is a project at Fermilab that provides a highintensity neutrino beam used to study neutrino oscillations and to understand neutrinonucleus weak force interactions. The protons are accelerated to about $120 \mathrm{GeV}$ and then impinge on the graphite target, producing secondary particles, mostly mesons. The NuMI target is designed to be about 1.2 meters long, two average nuclear interaction lengths to prevent the parent mesons of neutrinos from being re-absorbed by the target. Then, the magnetic field in the target hall focus the secondary hadrons, comprised of pions, kaons, and others into a narrow secondary beam. These remaining pions and kaons travel through the decay pipe and decay mostly to neutrinos and muons. The absorber stops most of the hadrons, and the rocks behind the absorber stop most of the muons, so then only neutrinos exit the end of this facility. (Fig. 1.3). We firstly run the NOvA experiment in $\nu$ mode, which means most of the secondary hadrons are positive pions and positive kaons whose 
decays result in neutrinos. Negative mesons that produce anti-neutrinos are bent away from the baseline in the horns of the beam-line.

The primary beam intensity for NuMI was designed to deliver about $6.0 \times 10^{20}$ protons on target (POT) per year by assuming a run of ten months with 60 percents of up time. The $700 \mathrm{~kW}$ of proton intensity is equivalent to about $5.0 \times 10^{13}$ POT per spill with a time window of 10 micro-seconds. And for each spill cycle, it takes 1.3 seconds [24, 74]. The following channels refer to the two major decays occurring in the decay pipe.

$$
\begin{aligned}
& \left.\pi^{+} \rightarrow \mu^{+}+\nu_{\mu} \text { (branch ratio } 99.9 \%\right) \\
& K^{+} \rightarrow \mu^{+}+\nu_{\mu}(\text { branch ratio } 63.6 \%)
\end{aligned}
$$

For an angle $\theta$ between the outgoing $\nu_{\mu}$ and its parent pion (Fig. 1.4), the energy of muon neutrino can be approximately described in Eq. 1.10.

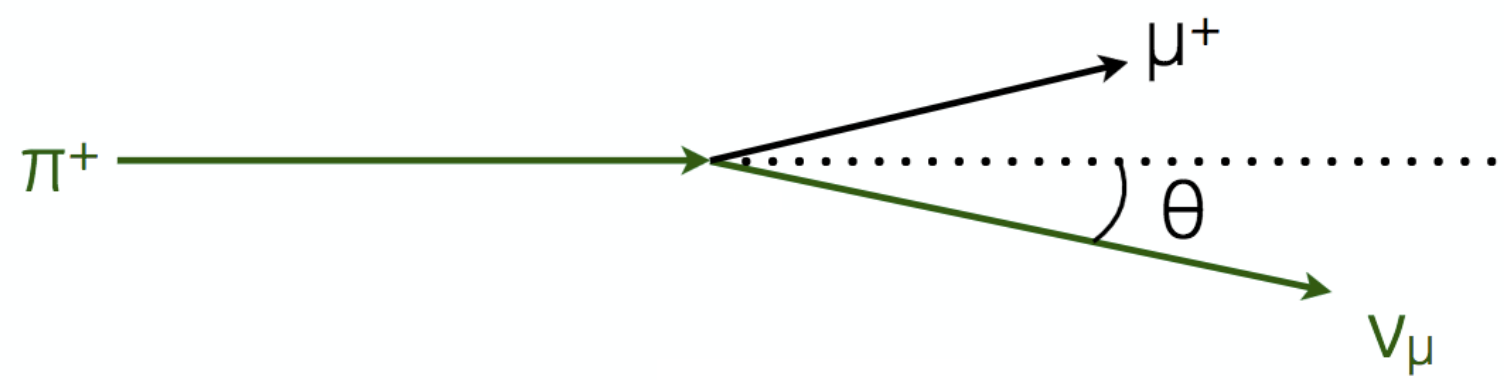

Figure 1.4. Off-axis muon neutrino from pion decay.

$$
E_{\nu} \approx \frac{1-\left(m_{\mu} / m_{\pi}\right)^{2}}{1+\gamma^{2} \tan ^{2} \theta} E_{\pi}
$$

A more intuitive relation can be found in Fig. 1.5. 


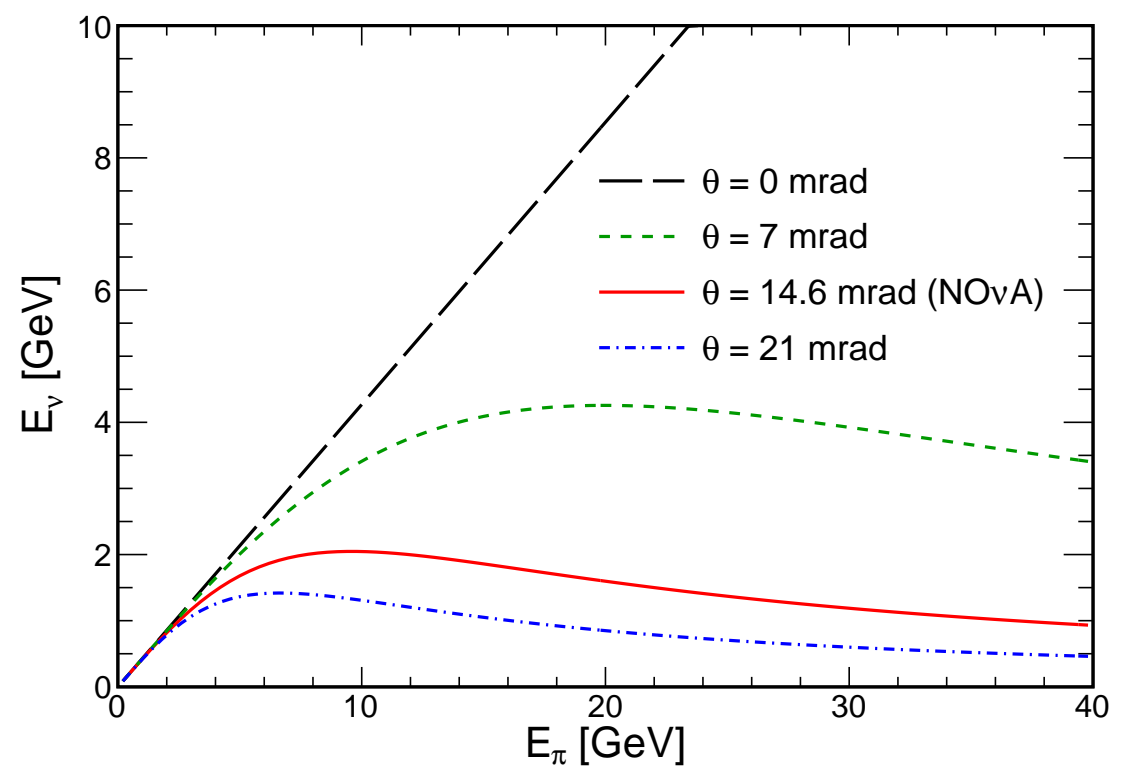

Figure 1.5. Muon neutrino energy vs its parent pion energy, [46]. 

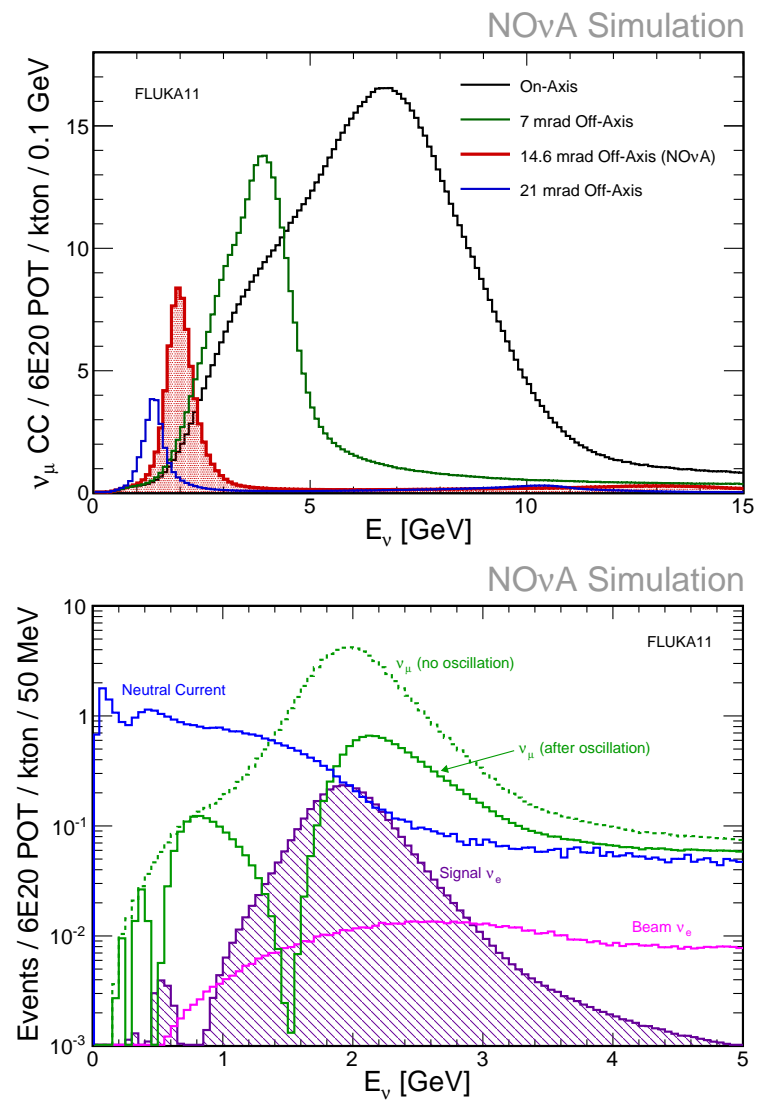

Figure 1.6. Off-axis angles comparison at far detector. We choose an off-axis angle to build the far detector, the full width at half maximum of the neutrinos spectrum peak is optimal at $14.6 \mathrm{mrad}$ off-axis. We also considered the ratio of signal $\nu_{e}$ to neutral current background to make this decision as shown on the right plot $[46,80]$.

Both NOvA near detector and far detector are located at an angle 14.6 mrad off-axis with respect to the original direction of the parent pions. This far detector location provides more flux at the oscillation maximum and less background from neutral current than other off-axis angles (Fig. 1.6). And in the near detector, the incident neutrinos have the same kinematic properties as in the far detector. This design allows for the reduction of the systematic uncertainty during the background extrapolation in the far detector for oscillation analysis.

The map and locatons of near detector and far detector are shown in Fig. 1.7, the NOvA near detector is located at Fermilab with one kilometer downstream of the beam source and 100 meters underground while the NOvA far detector is located on the ground surface at 
Ash River, a beautiful and peaceful town to the south of the U.S. and Canada border in Minnesota.
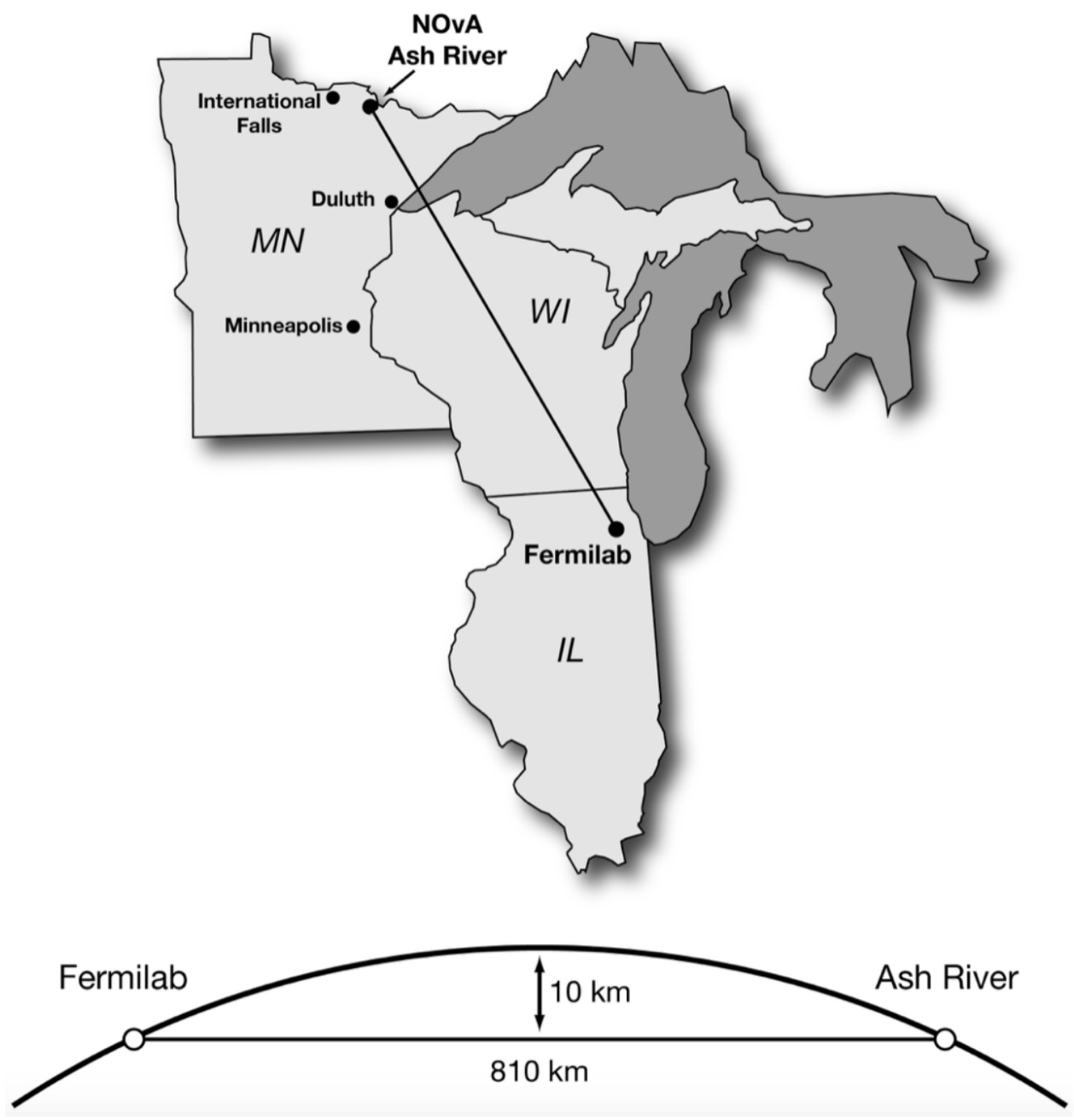

Figure 1.7. Locations of NOvA Near Detector and Far Detector. The NOvA near detector seats underground at Fermilab while the far detector is constructed on the surface at Ash River, MN.

While benefiting from the tremendous intensity of neutrino beam in the near detector, we noticed that the neutrino energy spectrum is much wider than in the far detector as shown in Fig. 1.8. The high-end tail of true neutrino energy can go up above $15 \mathrm{GeV}$. In the later chapters, I will show a data-driven method for high energy flux constraint. 

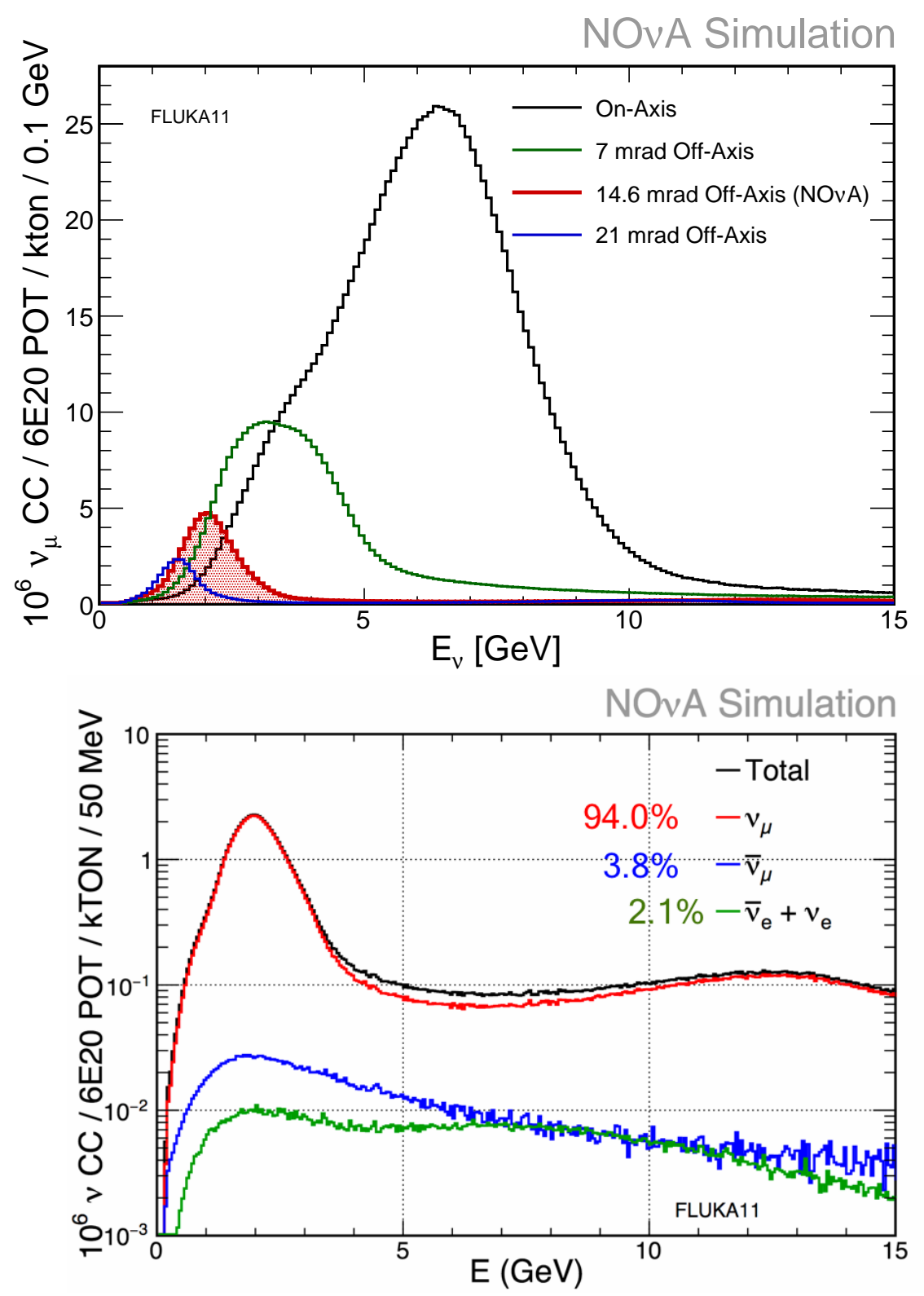

Figure 1.8. Muon neutrino energy spectrum at near detector [46] and the components of each type of neutrinos. 


\subsection{The NOvA detectors}

The NOvA detectors are designed to optimize the electron detection and measurement of its energy.

The NOvA near detector has dimensions of $4.2 \mathrm{~m} \times 4.2 \mathrm{~m} \times 15.8 \mathrm{~m}$. It is made of polyvinyl chloride (PVC) extrusion box (30\% of total mass) filled with 220 tons of liquid scintillator (about $65 \%$ of total mass). It is located $1 \mathrm{~km}$ from the beam source, about 100 meters underground. We had mixed titanium dioxide into PVC in order to enhance the inner reflectivity of scintillation light to $92 \%$ [19].

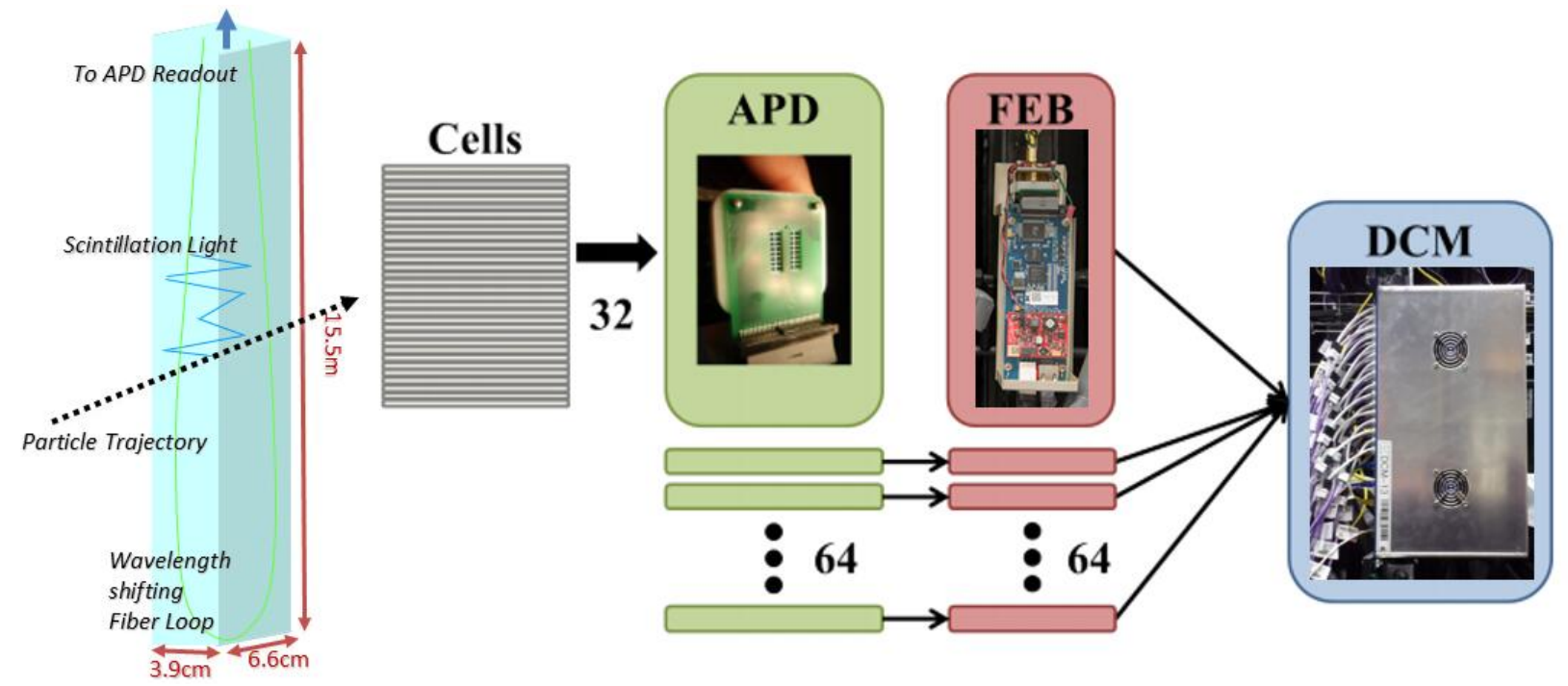

Figure 1.9. Structure of NOvA detectors. The scintillation light is generated by charged particles and collected by a looped wavelength-shifting fiber in each cell. Each avalache-photodiode (APD) reads out 32 cells and connects to a front-end-board (FEB). The FEB digitizes the readout from APD and sends the signal to a data-concentratormodule (DCM) [105, 107].

Figure 1.9 shows the structure of NOvA detectors. For each cell, the width is $39.3 \mathrm{~mm}$, with $66.1 \mathrm{~mm}$ in depth, the gap between planes is $4.8 \mathrm{~mm}$ on average, while gap between cells in a single plane is around $3.3 \mathrm{~mm}$. More technical details can be found in [92]. One looped wavelength-shifting fiber is inserted in each cell, both ends of each fiber are connected into a single Avalanche Photodiode (APD) pixel [21]. 
Besides the PVC planes, the near detector is also outfitted with a muon catcher at the downstream end. The muon catcher, just as the name implies, has steel plane inserted into each contiguous PVC plane, so that it can stop the through-going muons travel out of the scintillator part of the detector.

\begin{tabular}{|l|c|c|}
\hline & Far detector & Near detector \\
\hline Plane Size & $15.6 \mathrm{~m}$ by $15.6 \mathrm{~m}$ & $4.2 \mathrm{~m}$ by $4.2 \mathrm{~m}$ \\
\hline Detector Length & $60.0 \mathrm{~m}$ & $15.8 \mathrm{~m}$ \\
\hline Plane Number & 896 & 214 \\
\hline Channel Number & 344064 & 20192 \\
\hline Mass & $\sim 14 \mathrm{kTon}$ & $\sim 300$ Ton \\
\hline Diblocks & 14 & $3+$ muon catcher \\
\hline
\end{tabular}

Table 1.1. Size Comparison of Far Detector and Near Detector

The far detector has the same structure but bigger size in scale and without muon catcher. Full comparison of near detector and far detector can be found in Tab. 1.1.

We use mineral oil as the solvent of the liquid scintillator. The content of the scintillant pseudocumene is about five percent in the fluid. Tiny amount of PPO and bis-MSB are used for wave-shifting purpose. Both anti-static and antioxidant solution take about 0.001 percent in the fluid. So that the whole detector is built by using low Z material [75].

The longitudinal sizes of electromagnetic showers can be described in terms of the radiation length $X_{0}$, which depends on the material as shown in Eq. 1.11

$$
X_{0} \sim \frac{716.4 A}{Z(Z+1) \ln (287 / \sqrt{Z})} \mathrm{g} \mathrm{cm}^{-2}
$$

The structure of NOvA detectors provides $\mathrm{x}$ and $\mathrm{y}$ readout view in alternating planes [74]. The low $\mathrm{Z}$ material in the NOvA detector results in one radiation length to be approximately $38 \mathrm{~cm}$, about 6 planes in z direction. Thus a $1 \mathrm{GeV}$ electron resulting from the neutrino interaction in the NOvA detectors would create an average of 2.5 meter long electromagnetic shower crossing 40 planes in z direction [21]. The $3.9 \mathrm{~cm} \times 6.6 \mathrm{~cm}$ cell cross-section provides 
sufficient spacial resolutions of NOvA to understand the details of any electromagnetic shower pattern. The Molière radius of this type of electromagnetic shower encompasses about 2-3 cells in both vertical and horizontal radial dimensions [74].

This low-Z material and fine cell structure ensure the NOvA detectors to have good angular resolutions for neutrino-electron elastic scattering and lightweight dark matter search [102]. One can simply estimate the angular resolution of an electron induced shower by using Eq. 1.12.

$$
\text { resolution }(\theta) \sim \frac{R(\text { Molière })}{X_{0}} \sim Z \frac{\text { mrad }}{\sqrt{E / G e V}}
$$

In this section, I summarized the main structure and material of the NOvA detectors, so that when a charged particle passing by, the scintillation light is generated. Next I will describe the process about how the light is handled by the readout system. 


\subsection{Data Acquisition}

NOvA uses APD as the photon-sensor to convert the shifted scintillation light into electrical signals. The high quantum efficiency of an APD ensure sufficient photoelectrons been collected while a normal muon track passing at the further ends of cells with respect to the APD readout. Also the size of APD is small enough to be installed on high dense scintillator detector such as the far detector that NOvA has. The APD is cooled by thermoelectric cooler which turn the APD temperature to $-15 \mathrm{C}$. At $-15 \mathrm{C}$, noise was reduced so that the signal to noise ratio at the furthest end of each detector cell is about 10:1. At this temperature, the bulk dark current of APDs was lower than 0.3pA, which is the best limit on the output that we can reach.

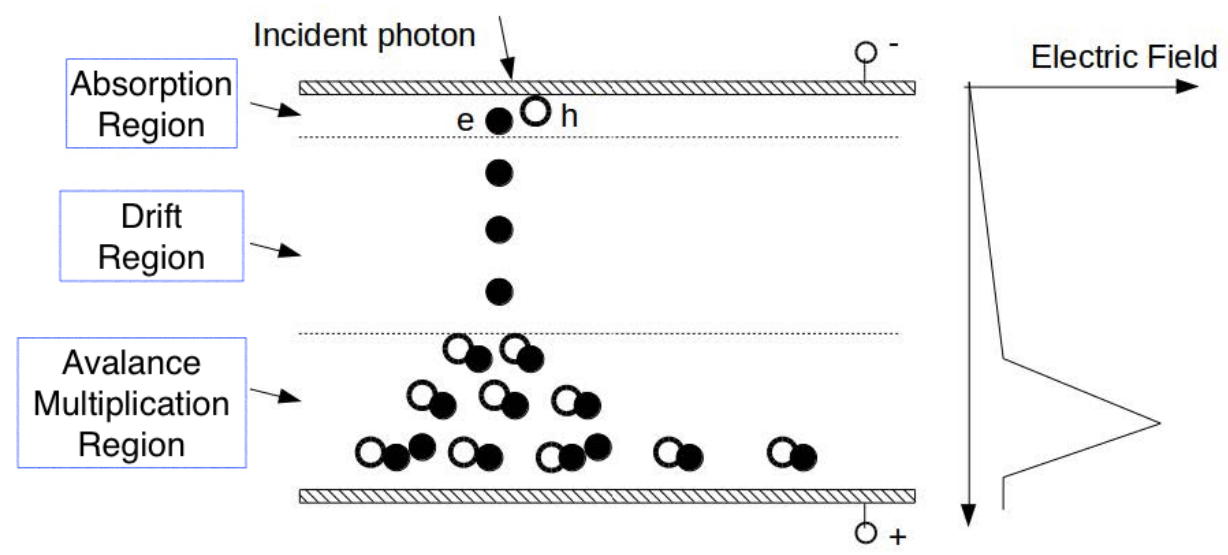

Figure 1.10. The diagram of APD structure. The curve on the right shows the strength of electric field as a function of drifting depth. The electrons in the multiplication region are driven by the strong electric field, which is created by high bias voltage of $425 \mathrm{~V}$ across the APD [104].

The avalanche process for an APD starts from the following: When a photoelectron enters the semiconductor layer of an APD, the energy is absorbed to produce electron-positive hole pairs in the high voltage electric field. And the charged particles continue to generate more electron-positive hole pairs resulting in avalanche multiplication [104]. Those electrons move towards the corresponding $\mathrm{PN}$ junctions and form a pulse used on input to the next electronic 
device, ASIC. ASIC shapes and amplifies the pulse and passes it to analog-to-digit converter $(\mathrm{ADC})$.

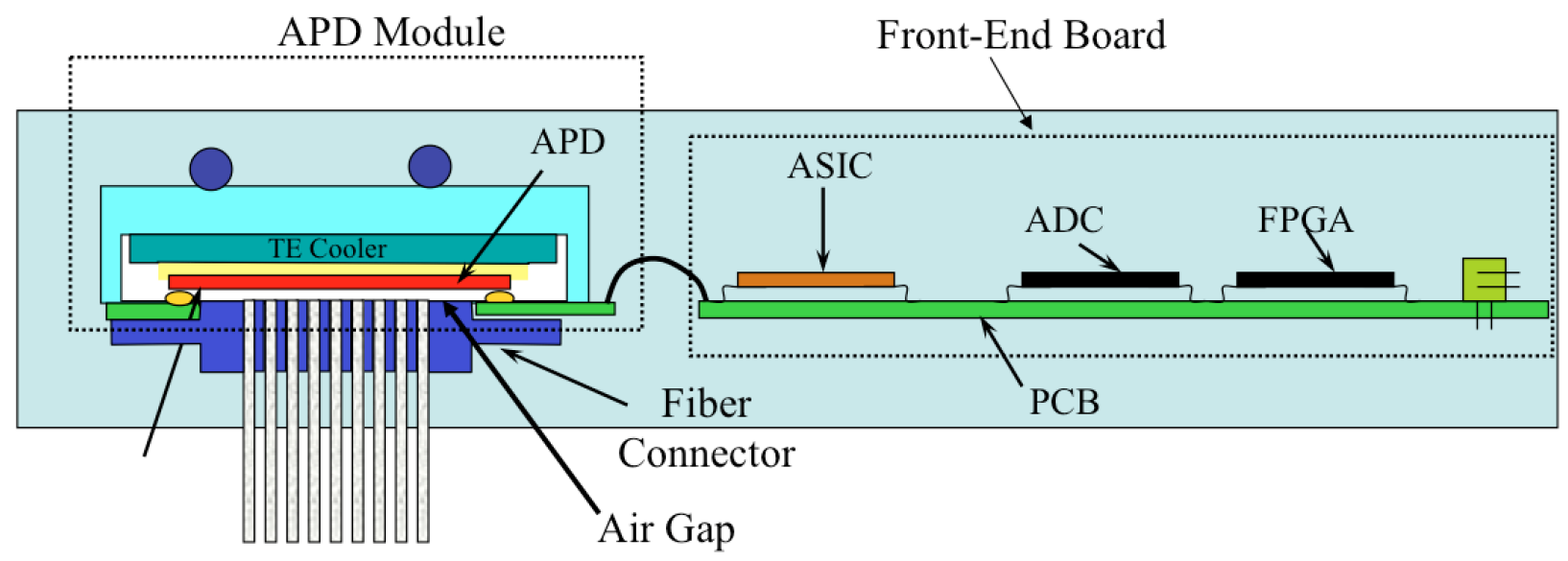

Figure 1.11. The readout interface.

To select the signal, the ASIC removes the low frequency and high frequency noise, forms a shaped pulse with rise time $\left(t_{r}\right)$ and fall time $\left(t_{f}\right)$ and transforms analog pulse to the digital form ADC. It describes the collected charge (q) as a function of time as in Eq. 1.13 [84].

$$
q(t) \propto \frac{t_{f}}{t_{f}-t_{r}} \times\left(e^{-\frac{t}{t_{f}}}-e^{-\frac{t}{t_{r}}}\right)
$$

Then an algorithm called dual correlated sampling compares the current ADC value $\left(\mathrm{ADC}_{i}\right)$ to the $\mathrm{ADC}$ value of three sampling times before $\left(\mathrm{ADC}_{i-3}\right)$. If $\mathrm{ADC}_{i}-\mathrm{ADC}_{i-3}$ $>4 \sigma_{\text {noise } A D C}$, then the signal is recorded as a hit and the information is sent to a data concentrator module (DCM). Then, we time-stamp and store every hit in the deep buffers. Each five milliseconds period interval, a data concentrator module transfers the accumulated data to a single buffer node. 


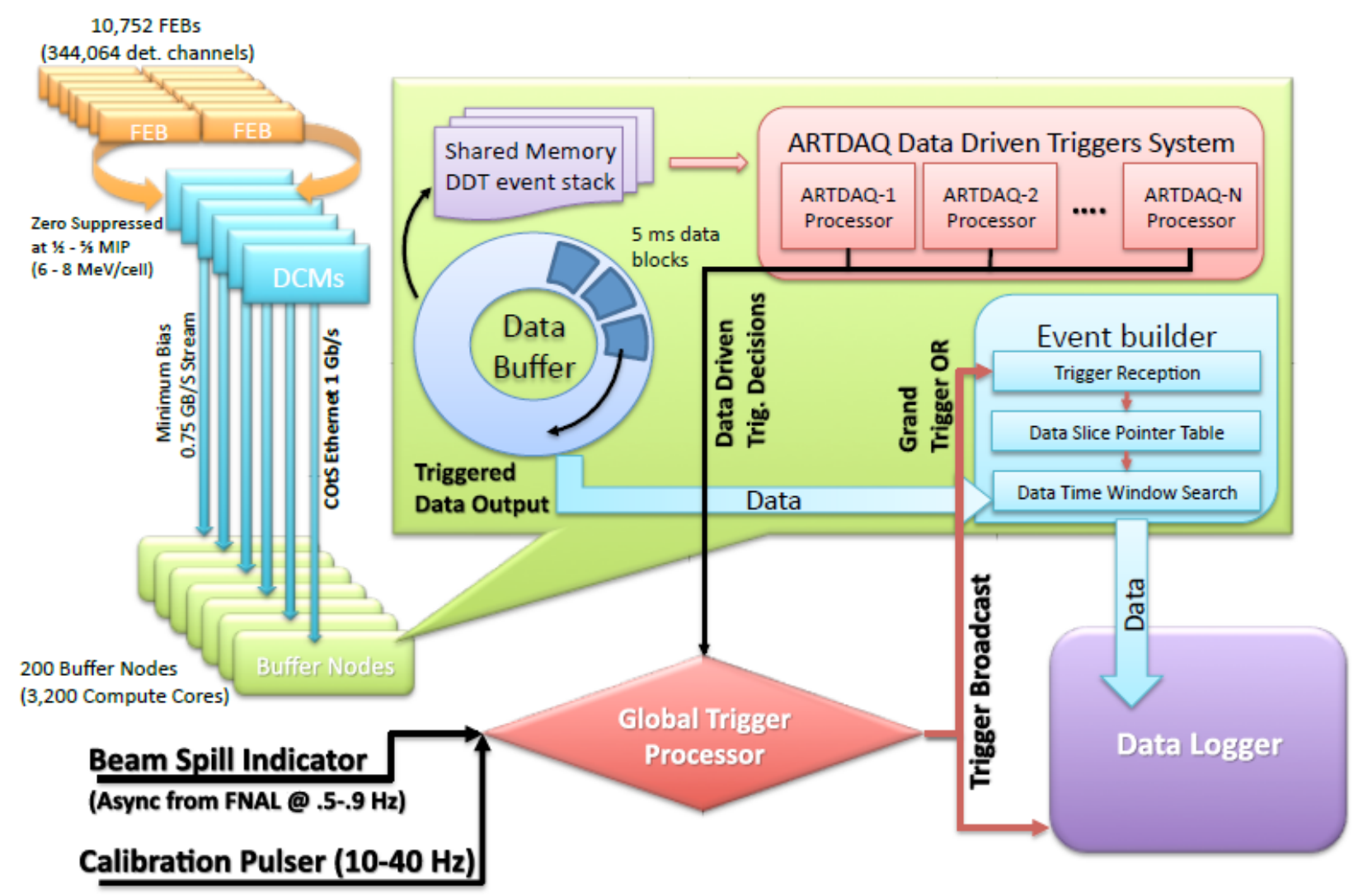

Figure 1.12. The logic structure of DAQ system, diagram is originally from Ref. [23,54].

The global trigger system shown in Fig. 1.12 provides the time window and the triggers with the corresponding start time to buffer nodes. After the trigger processor compares the stored hits with the beam spill information and other triggering conditions [15], the data is selected and recorded as a raw root file in the data logger system. 


\section{CHAPTER 2}

\section{Theoretical Background}

The Weak Interaction is one of the four fundamental forces of nature, and as such their elucidation is essential for understanding both microscopic and macroscopic phenomena. Central to the understanding of the weak interactions is the neutrino. The neutrino is the only fundamental particle whose interactions are dominated by the Weak Interactions. The neutrino interactions play a central role in nuclear transitions, energy transport in stars, Supernovas, and the evolution of the Universe.

The Glashow-Salam-Weinberg (GSW) model unifies under one theory the weak and electromagnetic interactions. This model's first spectacular success was the prediction of neutral currents. The existence of neutral currents was not even suspected, but the prediction was soon veried by the observation of anti muon neutrino electron elastic scattering in the Gargamelle experiment in December 1972 [64]. More than ten years later the models predictions were further verified by discovery of the W-bosons and the Z-boson in 1983 . The "coup de grace" for what we now call the Standard Model was the more recent discovery of the Higgs particle in July of 2012. Given that the neutrino plays such an important role in the weak interactions the study of its properties is as important as that of the Higgs-boson and may even provide a better understanding of the nature of the Higgs-boson. 


\begin{tabular}{c|c|c}
\hline Process & t-channel & s-channel \\
\hline$\nu_{\mu}+e \rightarrow \nu_{\mu}+e$ & $\mathrm{Z}$ & $\mathrm{N} / \mathrm{A}$ \\
$\bar{\nu}_{\mu}+e \rightarrow \bar{\nu}_{\mu}+e$ & $\mathrm{Z}$ & $\mathrm{N} / \mathrm{A}$ \\
$\nu_{e}+e \rightarrow \nu_{e}+e$ & $\mathrm{~W}, \mathrm{Z}$ & $\mathrm{N} / \mathrm{A}$ \\
$\bar{\nu}_{e}+e \rightarrow \bar{\nu}_{e}+e$ & $\mathrm{Z}$ & $\mathrm{W}$ \\
$\nu_{\mu}+e \rightarrow \nu_{e}+\mu$ & $\mathrm{W}$ & $\mathrm{N} / \mathrm{A}$ \\
$\bar{\nu}_{e}+e \rightarrow \bar{\nu}_{\mu}+\mu$ & $\mathrm{N} / \mathrm{A}$ & $\mathrm{Z}$ \\
\hline
\end{tabular}

Table 2.1. W and Z exchange in t-channel and s-channel.

The contributions of $\mathrm{W}$ and Z bosons' exchange in the crossed channel (t-channel) and direct channel (s-channel) can be found in Table. 2.1. I will focus on the $\nu_{\mu}-e$ scattering in the following sections.

\subsection{Neutrino Electron Elastic Scattering}

The present study will focus on the process where a muon-neutrino or an electron-neutrino scatter off an electron in the NOvA near detector (Fig. 2.1). Neutrino-electron elastic scattering has been the traditional method to study the neutral current weak interactions. The reason being that it is a purely weak interaction absent of the larger uncertainties that would arise if the strong interactions were involved. Furthermore, the characteristics of the neutrino beam from the NuMI facility can be very well constrained by using this channel [102]. This is because that the process through which they are produced results in a very forward and clean electromagnetic shower. 

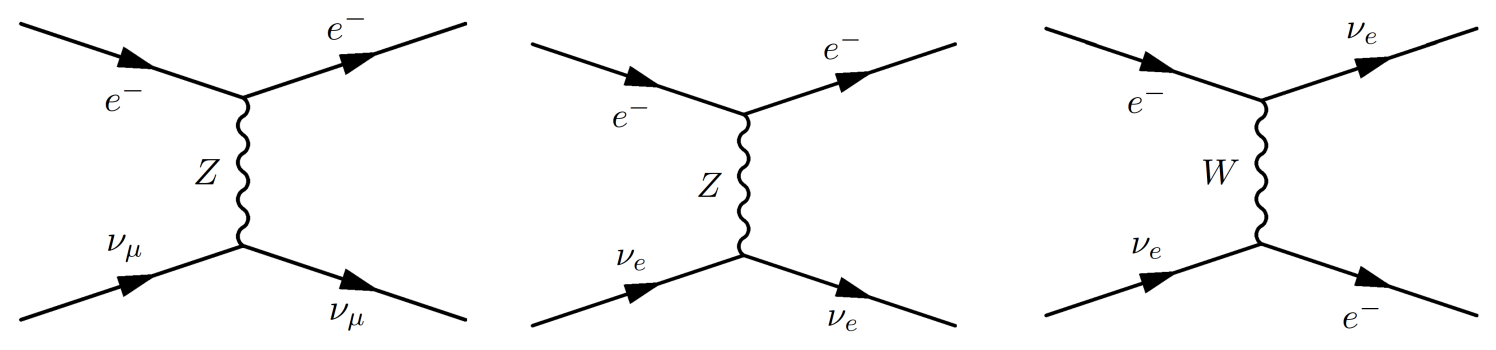

Figure 2.1. The lowest order Feynman diagrams of the $\nu$-e elastic scatterings.

A fundamental variable in any 2-to-2 scattering process is the center of mass energy of the particles, $\sqrt{s}$. The variable, $\mathrm{s}$, is called the Mandelstam variable and is defined by,

$$
s=\left(p_{\nu_{i}}+p_{e_{i}}\right)^{2}=m_{e}\left(m_{e}+2 E_{\nu}\right),
$$

where in the last equality we have evaluated s in the electron rest frame. Since the mean neutrino energy is much larger than the rest mass of electron, we use $s \approx 2 m_{e} E_{\nu}$. For energies much below the $\mathrm{W}$ and $\mathrm{Z}$ mass, we may use the four-point Fermi theory. The cross-section is approximately given by,

$$
\sigma=\frac{G_{F}^{2} s}{4 \pi}\left(C_{1}+\frac{1}{3} C_{2}+\frac{m_{e}^{2}}{s} C_{3}\right),
$$

where the $C_{1}, C_{2}$ and $C_{3}$ are constants for different $\nu$-e process as shown in Table. 2.2.

\begin{tabular}{cccc}
\hline Process & $C_{1}$ & $C_{2}$ & $C_{3}$ \\
\hline$\nu_{\mu}+e \rightarrow \nu_{\mu}+e$ & $\left(g_{V}+g_{A}\right)^{2}$ & $\left(g_{V}-g_{A}\right)^{2}$ & $g_{A}^{2}-g_{V}^{2}$ \\
$\bar{\nu}_{\mu}+e \rightarrow \bar{\nu}_{\mu}+e$ & $\left(g_{V}-g_{A}\right)^{2}$ & $\left(g_{V}+g_{A}\right)^{2}$ & $g_{A}^{2}-g_{V}^{2}$ \\
$\nu_{e}+e \rightarrow \nu_{e}+e$ & $\left(g_{V}+g_{A}+2\right)^{2}$ & $\left(g_{V}-g_{A}\right)^{2}$ & $\left(g_{A}+1\right)^{2}-\left(g_{V}+1\right)^{2}$ \\
$\bar{\nu}_{e}+e \rightarrow \bar{\nu}_{e}+e$ & $\left(g_{V}-g_{A}\right)^{2}$ & $\left(g_{V}+g_{A}+2\right)^{2}$ & $\left(g_{A}+1\right)^{2}-\left(g_{V}+1\right)^{2}$ \\
\hline
\end{tabular}

Table 2.2. The constants of coupling constants in different $\nu$-e scatterings, where $g_{A}=$ $-\frac{1}{2}, g_{V}=-\frac{1}{2}+2 \sin ^{2} \theta_{w} \quad$ [59]. 
The differential form of the $\nu_{\mu}$ - $e$ elastic scattering cross section can be written as:

$$
\left(\frac{d \sigma}{d T}\right)_{\text {Weak }}=\frac{G_{F}^{2} m_{e}}{2 \pi}\left[\left(g_{V}+g_{A}\right)^{2}+\left(g_{V}-g_{A}\right)^{2}\left(1-\frac{T}{E_{\nu}}\right)^{2}+\left(g_{A}^{2}-g_{V}^{2}\right) \frac{m_{e} T}{E_{\nu}^{2}}\right]
$$

where $G_{F}$ is the Fermi constant, $\mathrm{T}$ is the kinetic energy of a scattered electron, and $g_{V}$ and $g_{A}$ are coupling constants. A full derivation of the cross section can be found in Greiner's textbook [61] on weak interactions. The scattering angle $\theta$ between the outgoing electron and the incoming neutrino is given by [100], since the Compton formula for scattering angle in final states holds [25].

$$
\cos \theta=\frac{E_{\nu}+m_{e}}{E_{\nu}} \sqrt{\frac{T}{T+2 m_{e}}}
$$

Following some simple derivations for the small angle approximation $\theta \rightarrow 0$ and $\frac{m_{e}}{E_{\nu}} \rightarrow 0$ limit, we obtain:

$$
\begin{gathered}
2 m_{e} \cos ^{2} \theta=T \sin ^{2} \theta+\frac{m_{e}^{2}}{E_{\nu}^{2}} T+2 m_{e} \frac{T}{E_{\nu}}, \\
T \theta^{2}=2\left(1-\frac{T}{E_{\nu}}\right) m_{e},
\end{gathered}
$$

and so that $T \theta^{2}<2 m_{e}$, which provides an important kinematic feature for the events selection in the data analysis. Substitute Eq. 2.6 into $\frac{d \sigma}{d T}$, the differential cross-section can then be approximately written in terms of $\theta$ as well [25] by

$$
\left(\frac{d \sigma}{d \theta^{2}}\right)=\frac{G_{F}^{2} m_{e}^{2}}{\pi\left(\theta^{2}+2 m_{e} / E_{\nu}\right)}\left[\left(g_{V}+g_{A}\right)^{2}+\left(g_{V}-g_{A}\right)^{2}\left(\frac{\theta^{2}}{\theta^{2}+2 m_{e} / E_{\nu}}\right)+\left(g_{A}^{2}-g_{V}^{2}\right) \frac{2 m_{e}}{E_{\nu}^{2}\left(\theta^{2}+2 m_{e} / E_{\nu}\right)}\right]
$$

and

$$
\left(\frac{d \sigma}{d\left(T \theta^{2}\right)}\right)=\frac{G_{F}^{2} E_{\nu}}{4 \pi}\left[\left(g_{V}+g_{A}\right)^{2}+\left(g_{V}-g_{A}\right)^{2}\left(\frac{T \theta^{2}}{2 m_{e}}\right)^{2}+\left(g_{A}^{2}-g_{V}^{2}\right) \frac{m_{e}}{E_{\nu}}\left(1-\frac{T \theta^{2}}{2 m_{e}}\right)\right] .
$$




\subsection{Muon Neutrino Magnetic Moment}

Experimental evidence has yet to determine whether the neutrino is a Dirac or Majorana type fermion. A Majorana fermion is distinguished from a Dirac type fermion in that Majorana fermions are their own anti-particles. Clearly, only neutral fermions can be of Majorana type. Since the neutrino is neutral it may be Dirac or Majorana type. A Dirac neutrino can be distinguished from a Majorana type by their allowed electromagnetic properties. For example, we will see shortly that a Majorana neutrino cannot possess a diagonal dipole magnetic moment. In contrast, a magnetic moment for a Dirac neutrino is allowed as long as the neutrinos mass is nonzero. One other important phenomenological difference is that only a Majorana type neutrino can mediate neutrinoless double beta decay. Observation of neutrinoless double beta decay would be the telltale sign of a Majorana neutrino. One should keep in mind, however, that both types of neutrinos may exist in nature.

In minimum extensions of the Standard Model the magnetic moment of a Dirac neutrino is estimated to be of the order of $10^{-14} \mu_{B}[28,30]$, where $\mu_{B}$ is the Bohr magneton equals to $5.788 \times 10^{-5} \mathrm{eV} /$ Tesla. For Majorana neutrinos, on the other hand, much larger values for the transitional magnetic moment are possible [31,68].

Therefore, the experimental results of neutrino magnetic moment will be an important evidence for whether neutrino is Dirac or Majorana fermion and thus become the window to new physics [91].

\subsubsection{Magnetic Moment for Dirac Neutrinos}

In the Standard Model of particle physics the neutrinos are treated as massless fermions. However, it is trivial to extend the model to accommodate massive neutrinos without affecting the over structure of the theory. Several neutrino oscillation experiments like NOvA have detected neutrino flavor oscillations. The simplest explanation for neutrino oscillations is that the neutrinos have mass.

If a Dirac neutrino is massive then it may possess an intrinsic magnetic moment. When a Dirac neutrino exchanges a photon during the scattering process, it has to flip its helicity. 
For example, a left handed neutrino has to switch to the right handed neutrino, which is allowed in the minimum extension of Standard Model. In order to verify that, there must be a mass term in the amplitude $|A|$ of this cross-section. Within a minimum extension of Standard Model physics that allows the existence of right-handed neutrino, the Dirac neutrinos' electromagnetic properties can be described as

$$
\mu_{\nu_{i}}=\frac{3 m_{e} G_{F}}{4 \sqrt{2} \pi^{2}} m_{\nu_{i}} \simeq 3 \times 10^{-19}\left(\frac{m_{\nu_{i}}}{e V}\right) \mu_{B}
$$

Note that the magnetic moment of a Dirac neutrino is proportional to neutrino mass. Take $\nu_{\mu}$ for example, according to PDG 2014, $m_{\nu_{\mu}}<0.19 \mathrm{MeV}$ for $90 \%$ conference level. Then $\mu_{\nu_{\mu}}<5.7 \times 10^{-14} \mu_{B}$, which value is too tiny to be observed in the NOvA near detector.

In fact, such a tiny value of muon neutrino magnetic moment is impossible to be measured by any direct methods in any experiments in the next several decades. The magnetic properties of Dirac neutrino might cause effects in radiation, atomic scattering and thus effect the oscillation results.

The radiative decay rate for

$$
\nu_{i} \rightarrow \nu_{j}+\gamma
$$

is given by $[60]$

$$
\Gamma_{i j}^{\gamma} \approx 5.3\left(\frac{\mu_{i j}}{\mu_{B}}\right)^{2}\left(\frac{m_{i}^{2}-m_{j}^{2}}{m_{i} \times 1 e V}\right)^{3} s^{-1} .
$$

This process is determined by the neutrino mass ordering and the transitional magnetic moment $\mu_{i j}$. Project- 8 experiment is aiming to measure the neutrino mass directly from the precise beta electron spectroscopy [16], by such kind of evidence, we can safely derive the upper limit of a Dirac neutrino's magnetic moment and thus form a radiative term correction for the Dirac neutrino involved interactions.

\subsubsection{CPT Properties of EM Field for Majorana Neutrinos}

A Majorana Neutrino is its own antiparticle and it has a set of two spin states. it goes back to itself under the CPT transformation with a 180 degrees rotation [65]. 
Table 2.3. The C, P, T properties for electromagnetic field.

\begin{tabular}{|l|c|c|c|c|}
\hline Transformation & $\vec{A}$ & $\phi$ & $\vec{B}$ & $\vec{E}$ \\
\hline Parity (Space Inversion) & - & + & + & - \\
\hline Charge Conjugation & - & - & - & - \\
\hline Time Reversal & - & + & - & + \\
\hline CPT & - & - & + & + \\
\hline
\end{tabular}

Suppose $\mu_{M}$ is a Majorana neutrino's diagonal magnetic dipole moment and $\epsilon_{M}$ represents its diagonal electric dipole moment. Then in a classic electromagnetic field, the interaction energy can be written as in Eq. 2.12.

$$
E_{\text {int }}=-\mu_{M} \vec{s} \cdot \vec{B}-\epsilon_{M} \vec{s} \cdot \vec{E}
$$

Then, according to the CPT properties of B field and E field shown in Table 2.3, if CPT is still invariant, one should have

$$
E_{C P \text { Tint }}=\mu_{M} \vec{s} \cdot \vec{B}+\epsilon_{M} \vec{s} \cdot \vec{E}=E_{i n t}=-\mu_{M} \vec{s} \cdot \vec{B}-\epsilon_{M} \vec{s} \cdot \vec{E}
$$

That indicates that under CPT invariance, $\mu_{M}=0$ and $\epsilon_{M}=0$. Then we get the conclusion that if Majorana neutrino has magnetic moments, then it has to switch its flavor during magnetic interaction.

\subsubsection{Hypothesis of Large Transition Magnetic Moments for Majorana Neutrinos}

The origin of a Majorana Neutrino's magnetic moment arises from SU(2) symmetry and thus bypass the constraint from neutrino's mass [20,31]. It is possible that $\mu_{\nu} \approx \mathcal{O}\left(10^{-9} \mu_{B}\right)[31$, 35], a value which could be tested in the NOvA near detector by using data from three years of running at NuMI designed intensity $\left(1.8 \times 10^{21}\right.$ POT $)$. 


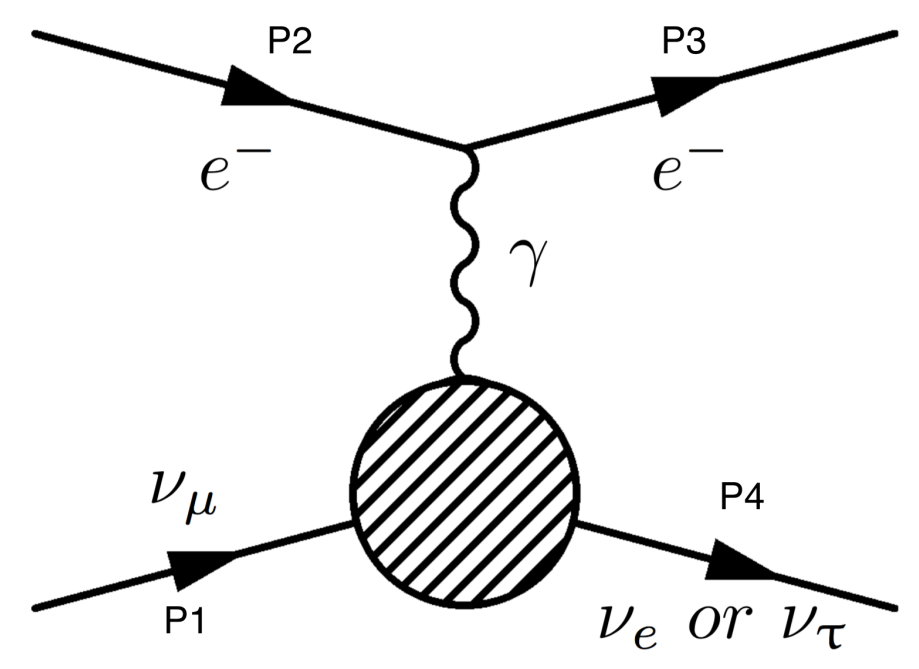

Figure 2.2. Magnetic scattering due to transitional magnetic moments. The CPT invariance force a Majorana neutrino of a given spin to rotate into one of another two flavor with different spin.

When a Majorana muon neutrino scatters off a free electron switches its flavor into one of the other two flavors (Fig. 2.2) due to a large transition magnetic moment. The scattered electron has a kinetic energy $T$, we keep this notation from now on. The bulb included all the possible Feynman diagrams in Fig. 2.2. By looking at the incoming and outgoing states, we can write the spin-averaged matrix element squared term as

$$
\left\langle|\mathcal{M}|^{2}\right\rangle=\frac{32 \pi^{2} \alpha^{2} \mu_{\nu}^{2}}{m_{e}^{2}} \frac{\left(p_{1} \cdot p_{2}\right)\left(p_{1} \cdot p_{3}\right)}{p_{1} \cdot p_{4}} .
$$

The Mandelstam variable $\mathrm{t}$ is given by the transferred momentum squared $Q^{2}$, and

$$
t=Q^{2}=-q^{2}=\left(p_{1}-p_{4}\right)^{2}=-2 p_{1} \cdot p_{4}=-2 m_{e}\left(T-m_{e}\right) .
$$

These $t, s$ and inelasticity $y\left(y \equiv \frac{T}{E_{\nu}}\right)$ are invariant under Lorentz transformations [55]. So that the differential cross section in terms of $\mathrm{s}$ and $\mathrm{t}$ can be described as Eq. 2.16 in Ref. [26,67].

$$
\frac{d \sigma}{d t}=\pi r_{e}^{2} \frac{s+t-m_{e}^{2}}{t\left(s-m_{e}^{2}\right)}\left(\frac{\mu_{\nu}}{\mu_{B}}\right)^{2}
$$


The differential cross section in terms of $\mathrm{T}$ due to magnetic scattering can be found in Eq. 2.17.

$$
\left(\frac{d \sigma}{d T}\right)_{E M}=\pi r_{e}^{2}\left(\frac{1}{T}-\frac{1}{E_{\nu}}\right)\left(\frac{\mu_{\nu}}{\mu_{B}}\right)^{2}=\frac{\pi \alpha^{2}}{m_{e}^{2}}\left(\frac{1}{T}-\frac{1}{E_{\nu}}\right)\left(\frac{\mu_{\nu}}{\mu_{B}}\right)^{2}
$$

Here $\alpha$ is the fine structure constant and $r_{e}$ is the classical electron charge radius.

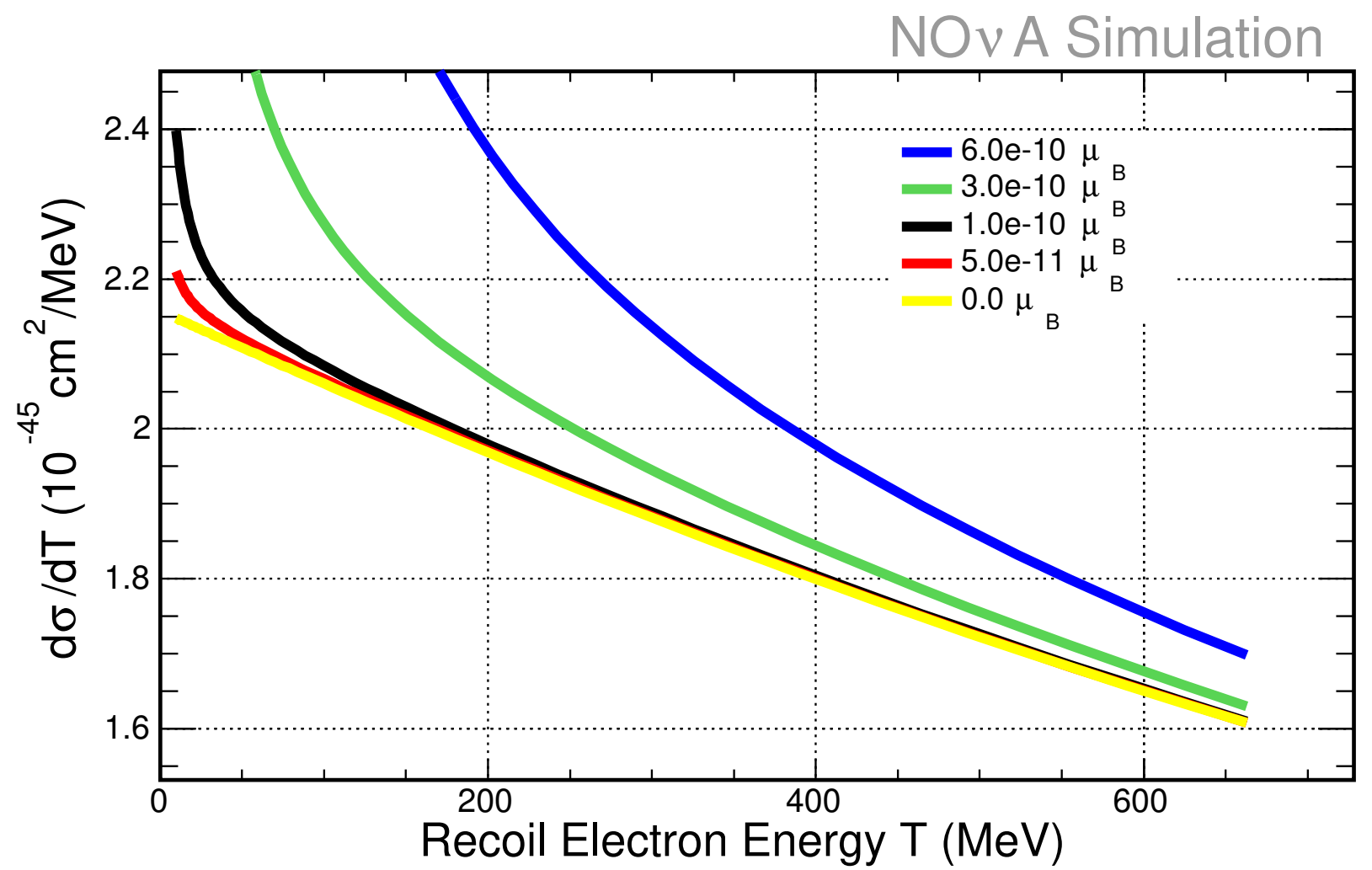

Figure 2.3. The differential cross-section for $\nu_{\mu}$-e scattering for various values of neutrino magnetic moment by using Eq. 2.3 and Eq. 2.17.

Figure 2.3 shows the weak and electromagnetic cross-sections for $\nu_{\mu}$ scattering off electrons for various values of the neutrino magnetic moment (NMM) as a function of the electron recoil energy $T$.

The divergence between the purely weak standard model process and the combined weak and possible electromagnetic processes for decreasing $T$ is the signature we seek for evidence of a large transitional magnetic moment for muon neutrino that produced in the NuMI 
facility.

\begin{tabular}{llll}
\hline Experiments & Year & Results at 90\% C.L. & Notes \\
\hline E-734 & 1989 & $<0.85 \times 10^{-9} \mu_{B}$ & $159.5 \nu_{\mu^{-}} e, 96.7 \overline{\nu_{\mu}}-e$. \\
LAMPF & 1993 & $<0.74 \times 10^{-9} \mu_{B}$ & $236 \nu_{e^{-}}, 284 \nu_{-} e, 295$ data fit. \\
CHARM II & 1995 & $<3 \times 10^{-9} \mu_{B}$ & $2677 \nu_{\mu^{-}} e, 2752 \overline{\nu_{\mu}}-e$. \\
LSND & 2001 & $<0.68 \times 10^{-9} \mu_{B}$ & $191 \nu_{e^{-}} e, 6 \nu_{\mu^{-}} e(24$ pred. $)$. \\
MiniBooNE & 2008 & $<1.27 \times 10^{-9} \mu_{B}$ & $15.3 \nu_{\mu^{-}} e, 4.6$ weak ES, 10.7 EM ES. \\
\hline
\end{tabular}

Table 2.4. List of previous experiments that provided an upper limit of muon neutrino magnetic moment.

The current published upper limits for the $\nu_{\mu}$ magnetic moment are from several independent experiments as shown in Table. 2.4, LSND $\left(\mu_{\nu_{\mu}}<6.8 \times 10^{-10} \mu_{B}\right)$ [17], LAMPF $\left(\mu_{\nu_{\mu}}<\right.$ $\left.7.4 \times 10^{-10} \mu_{B}\right)$ [10], CHARM II $\left(\mu_{\nu_{\mu}}<3 \times 10^{-9} \mu_{B}\right)$ [97] and BNL-E734 $\left(\mu_{\nu_{\mu}}<8.5 \times\right.$ $10^{-10} \mu_{B}$ ) [7]. All these results provide upper limits at an order of $10^{-9} \mu_{B}$. However, the strictest limit from LSND has a questionable observed result (observed six events and predicted 24 events from SM) that far below the standard model predicted.

For both LSND and LAMPF, $\nu_{\mu}$-e elastic scattering was considered as the background events of $\nu_{e}$ interactions. While for BNL-E734, both $\nu_{\mu}$ and $\bar{\nu}_{\mu}$ were involved. We also notice that all the $\nu_{\mu}$ dominated experiments provided a large upper limit on the muon neutrino magnetic moment. For these reasons, we aim to estimate a more reliable upper limit for muon neutrino magnetic moments, or find some evidence for an non-zero hypothesis of muon neutrino magnetic moment by using a much more pure muon neutrino flux.

In fact, the $\mu_{\nu}$ shown in Eq. 2.17 represents the effective result of both magnetic and electric dipole moments [60]. Note that when we count events, the events caused by $\nu_{\mu}$ to $\nu_{e}$ transitional magnetic moment $\mu_{e \mu}$ is proportional to $\mu_{e \mu}^{2}$, and so does the case for $\nu_{\mu}$ to $\nu_{\tau}$ transition. And we will never know the final states of the outgoing neutrinos, so when we measure the transition magnetic moment of a single neutrino flavor, we actually measure the longest side of a geometrical right triangle (Fig. 2.4). Which means for each type of neutrinos, we have 


$$
\begin{aligned}
& \mu_{\nu_{\mu}}^{2}=\mu_{\mu \tau}^{2}+\mu_{e \mu}^{2}, \\
& \mu_{\nu_{e}}^{2}=\mu_{e \tau}^{2}+\mu_{e \mu}^{2}
\end{aligned}
$$

and

$$
\mu_{\nu_{\tau}}^{2}=\mu_{\mu \tau}^{2}+\mu_{e \tau}^{2}
$$

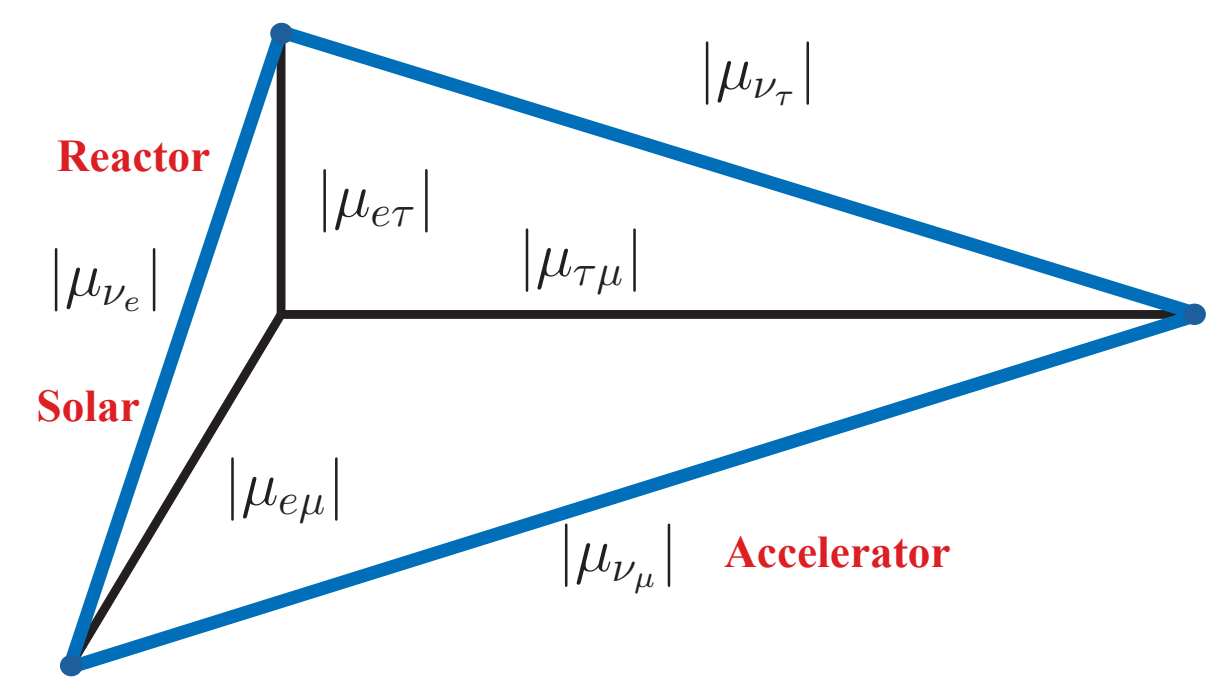

Figure 2.4. Triangle relations for Majorana neutrino magnetic moments. Plot is taken from Ref. [56].

If there are only three flavors of Majorana neutrinos, those three transition magnetic moments can form a triangular relation [56] as shown in Fig. 2.4. Reactor experiments such as GEMMA have provided the direct measurement of the $\mu_{\nu_{e}}$ upper limits at the order of $10^{-11} \mu_{B}$ [29]. However, the $\mu_{\tau \mu}$ component shown in Fig. 2.4 [56] will be better measured in accelerator experiments like NOvA and DUNE.

If limits are set, we can combine the results from reactor experiments to do a joint analysis so that constrain the tau neutrino magnetic moment by using $\left|\mu_{\nu_{\tau}}\right|^{2} \leq\left|\mu_{\nu_{\mu}}\right|^{2}+\left|\mu_{\nu_{e}}\right|^{2}[56]$, thereby providing constraints on models of the early universe in section 2.3 . 


\subsection{The Impacts of NMM in Cosmology and Astrophysics}

Primordial nucleosynthesis and microwave background are some of the most important pieces of evidence for the current model of our universe [39]. There are many parameters involved in the numerical calculation for the prediction from this model.

Magnetic moments of neutrinos impact the final abundance of different isotopes at the end of the Big Bang nucleosynthesis (BBN), as Ref. [95] describes in detail. The NOvA experiment is capable of providing constraints of the muon neutrino magnetic moment between $10^{-10} \mu_{B}$ and $10^{-9} \mu_{B}$, which dramatically impacts the final results of big bang nucleosynthesis.

According to the standard Big Bang model, in the early universe, the energy density was dominated by photons and neutrinos. The photons that were cooled by the expansion of the universe behave as the main components of cosmic microwave background (CMB) with a temperature around $2.7 \mathrm{~K}$ in today's universe. In contrast, the neutrinos from that era end up with energy corresponding to

$$
T_{\nu}=(4 / 11)^{1 / 3} T_{\gamma} \approx 1.9 \mathrm{~K}
$$

which can only be computed but not measured directly. The neutrino-photon interactions within the Standard Model have been studied in Ref. [48,96]. However, since their effects associated with the bayon-to-photon ratio in the Big Bang era are too tiny to be calculated as numerical precisions in the final isotope abundance, we consider the $\nu-\gamma$ impacts can be negligible for now. And the neutrino-photon interactions through transitional magnetic moments is certainly a topic worthy to be studied in the future.

In the early universe, the Majorana neutrino magnetic moments impacts on the quantity $N_{\text {eff }}$ and thus cause the relativistic energy density $\rho_{\text {relativistic }}$ in thermal equilibrium different than in the Standard Model.

$$
\rho_{\text {relativistic }}=\frac{\pi^{2}}{15} T_{\gamma}^{4}\left[1+\frac{7}{8} N_{\text {eff }}\left(\frac{T_{\nu}}{T_{\gamma}}\right)^{4}\right]
$$


Referring to the value $N_{\text {eff }}=3.30 \pm 0.27$ quoted by the Planck collaboration, one can then compare the effects from different components of the Majorana neutrino magnetic moments (Fig. 2.5).

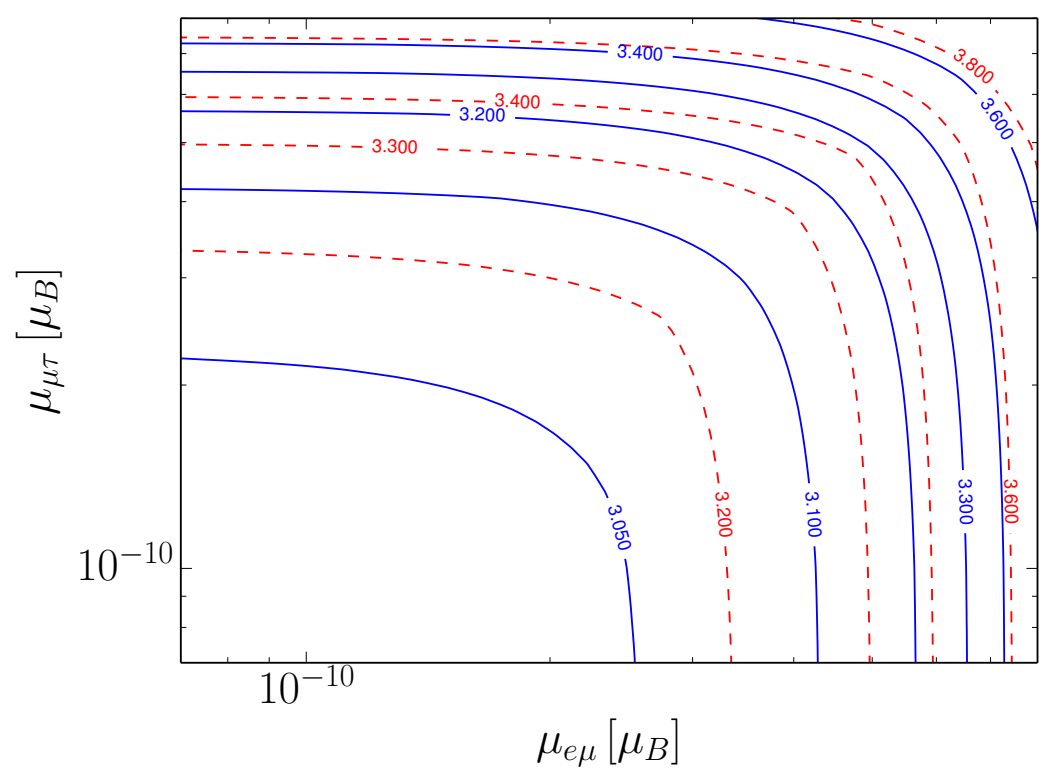

Figure 2.5. Contours of constant $N_{\text {eff }}$ in the $\mu_{\mu \tau}$ versus $\mu_{e \mu}$ plane. The solid contours correspond to $\mu_{e \tau}=10^{-10} \mu_{B}$ and the dashed contours correspond to $\mu_{e \tau}=4.9 \times 10^{-10} \mu_{B}$. This plot is taken from Ref. [95].

Neutrinos maintain the thermal equilibrium by weak interactions. The original neutron to proton ratio $n / p$ is determined when the neutrinos decoupled from thermal equilibrium. A large neutrino magnetic moment can enhance the magnetic interactions and change the neutrinos decoupling temperature with effects on the $n / p$ ratio. The magnetically scattering and annihilation from neutrinos modify the initial conditions of BBN and thus effect the relative entropy per baryon in the plasma. In the end, a larger initial entropy per baryon in the plasma causes increases in deuterium and ${ }^{3} \mathrm{He}$, and a decrease in ${ }^{7} \mathrm{Li}$. This primordial ${ }^{7} \mathrm{Li}$ abundance is inferred from spectroscopic observations of metal-poor halo stars [58]. The observational value of ${ }^{7} \mathrm{Li} / \mathrm{H}$ abundance $\left((1.6 \pm 0.3) \times 10^{-10}\right)$ is much lower than the theoretical prediction in standard BBN model. The source of this disagreement may come from either 
observation uncertainty or theoretical model or both. This unsolved puzzle can be fixed a little by setting up large values of neutrino magnetic moments (Fig. 2.6) and involve it into the calculation.

And in the NOvA experiment, the sensitive bound on muon neutrino magnetic moment in such range shown in Fig. 2.6 can be well examined. Therefore, a large muon neutrino transition magnetic moment might help fix the prediction of the relative final abundance of ${ }^{7} \mathrm{Li}$ while keeps the abundance of other isotopes within the uncertainties of observations. 


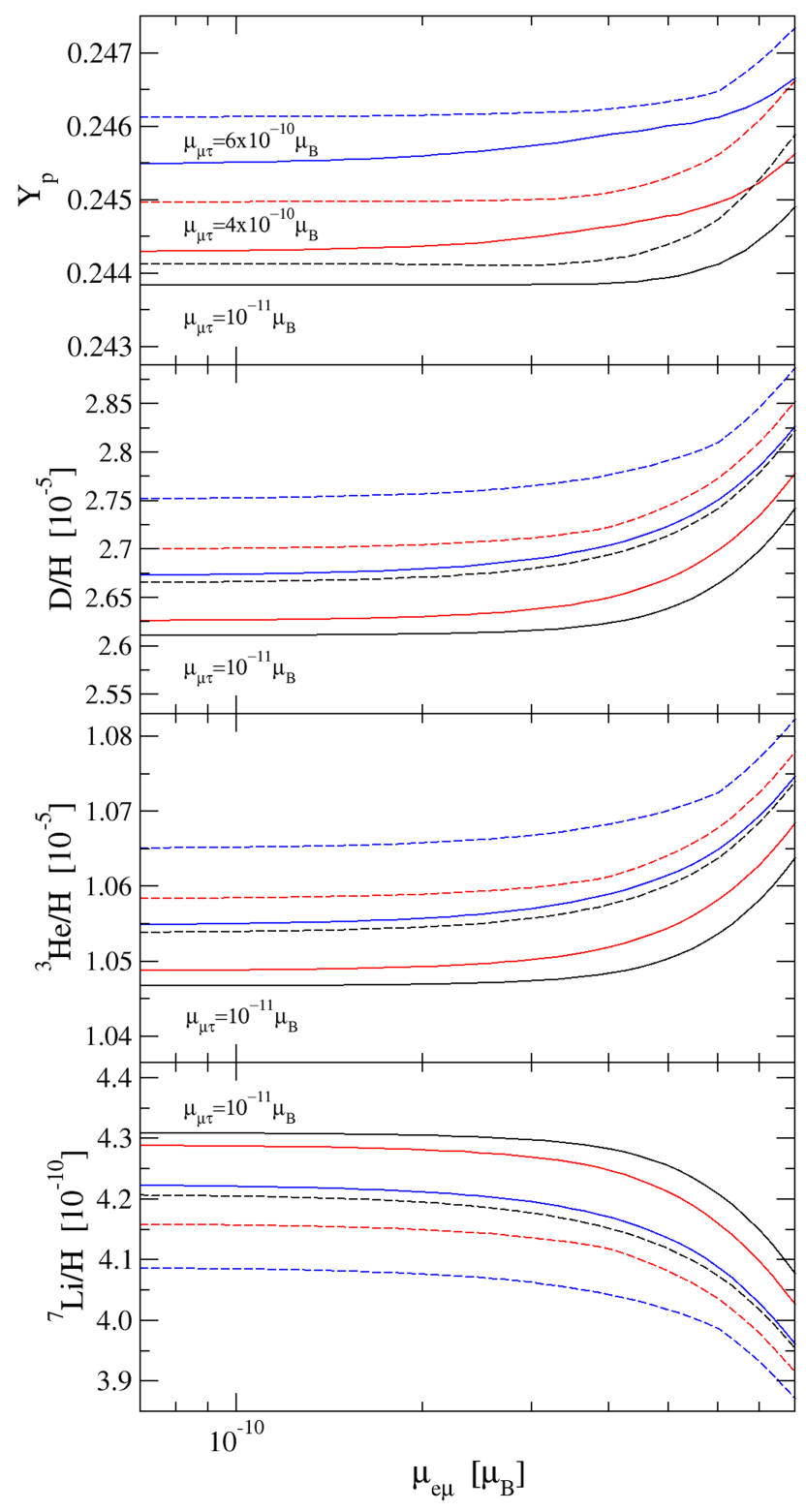

Figure 2.6. The change in primordial relative abundances of ${ }^{4} \mathrm{He}, \mathrm{D},{ }^{3} \mathrm{He}$, and ${ }^{7} \mathrm{Li}$ as a function of the transition neutrino magnetic moment $\mu_{e \mu}$. The solid lines show the results when $\mu_{e \tau}=10^{-11} \mu_{B}$ (black - $\mu_{\mu \tau}=10^{-11} \mu_{B}$, red $-\mu_{\mu \tau}=4 \times 10^{-10} \mu_{B}$, and blue $-\mu_{\mu \tau}=$ $\left.6 \times 10^{-10} \mu_{B}\right)$. The dashed lines are the results for $\mu_{e \tau}=6 \times 10^{-10} \mu_{B}$ with the colors representing the same values of $\mu_{\mu \tau}$ that they did in the solid case. The plot and caption details are from Ref. [95]. 
CHAPTER 3

Event Reconstruction and Classifier

\subsection{Monte Carlo Simulation}

The general simulation of NOvA experiment is using the GEANT4 software [11]. The generator input the neutrinos information from beam simulation and call the GENIE package $[13,14]$ for cross-section modeling. Then deliver the final states to GEANT4 package to realize the particles as Monte Carlo files.

For the neutrino beam simulation, two packages are used originally developed for the collider physics: FLUKA [36] and FLUGG [37]. Source of the neutrinos are the decays of pions and kaons produced in the proton on NuMI target collision. The decays are illustrated in Fig. 3.1. We use FLUKA to simulate meson production in the target while using FLUGG for the trajectories and decays of those mesons. 


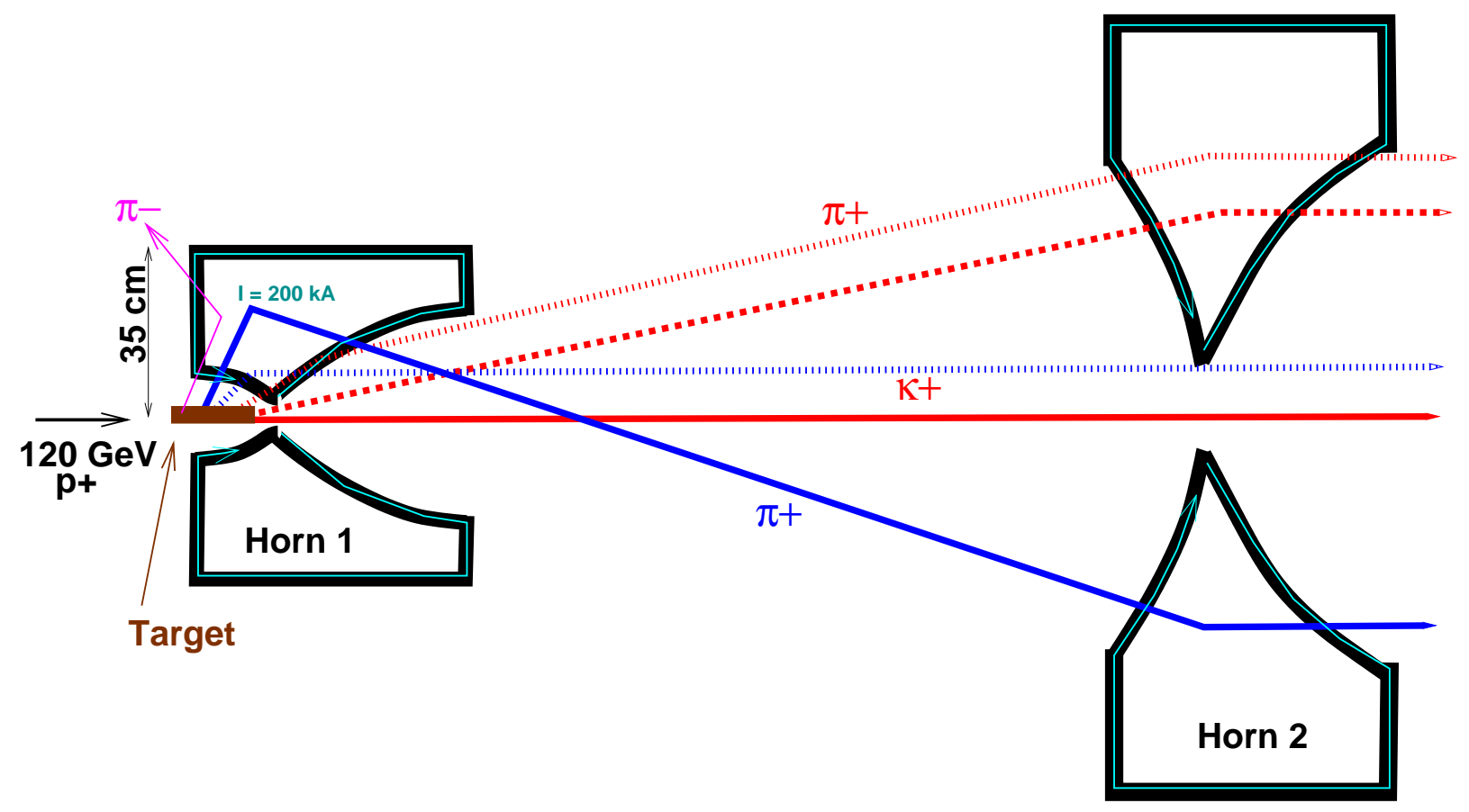

10 meters

Figure 3.1. The tracks of mesons in the magnetic horns, [4].

Then for the interaction part, we use GENIE package $[13,14]$ for neutrino interactions in the detector and CRY [63] for the cosmic rays that are mostly generated in the earth atmosphere. GENIE call the flux files and then input the true kinematic information of beam neutrino into the spline function of each interaction channel to produce the final states of each interaction. For the leptonic process like $\nu$-e elastic scattering, GENIE itself is good enough to simulate the cross section, but when hadronic process involved, GENIE will call the Pythia package [90] to describe the details of those hadronic showers. Then GENIE process the information of final states to GEANT4 package, and which generate the tracks, prongs and hit clusters in the detectors. And the description of detector material and structure are done by GDML files, written in the geometry description markup language developed in CERN. Take the near detector GDML files for example, one may use to the following command to check the simulated constituent material and isotope for the NOvA near detector. 


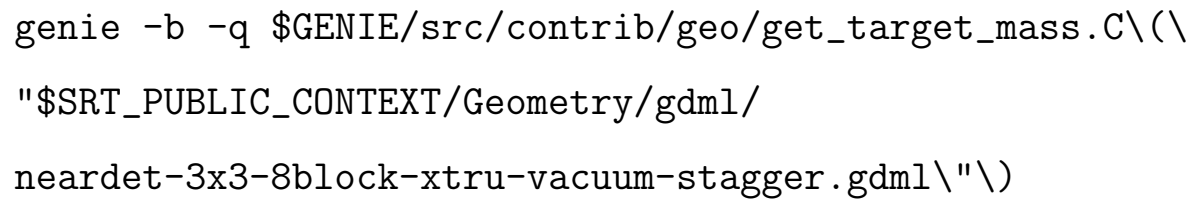

The results can be found in Tab. 3.1 and Tab. 3.2, note that most of the volume are the rocks in front of the detector.

\begin{tabular}{cc|c|cc}
\hline Index & Name & Volume $\left(\mathrm{m}^{3}\right)$ & Mass $(\mathrm{kg})$ & Mass $(\%)$ \\
\hline 0 & Vacuum & $2.56765 \mathrm{e}+09$ & $2.56765 \mathrm{e}-13$ & $9.78425 \mathrm{e}-24$ \\
2 & Granite & $9.71952 \mathrm{e}+08$ & $2.62427 \mathrm{e}+12$ & 100 \\
5 & Concrete & 82.3667 & 189443 & $7.2189 \mathrm{e}-06$ \\
7 & Scintillator & 154.254 & 132504 & $5.04918 \mathrm{e}-06$ \\
8 & PVC & 48.6708 & 72519.6 & $2.76342 \mathrm{e}-06$ \\
9 & Glue & 0.547541 & 733.705 & $2.79584 \mathrm{e}-08$ \\
13 & Steel & 10.8635 & 85387 & $3.25374 \mathrm{e}-06$ \\
\hline
\end{tabular}

Table 3.1. The material list in the NOvA near detector GDML files. 


\begin{tabular}{cccc|ccc}
\hline $\mathrm{Z}$ & $\mathrm{A}$ & $\mathrm{PDG}$ & Isotope & Volume $\left(\mathrm{m}^{3}\right)$ & Mass $(\mathrm{kg})$ & Mass $(\%)$ \\
\hline 1 & 1 & 1000010010 & $\mathrm{H}$ & 24.4289 & 22246.7 & $8.47727 \mathrm{e}-07$ \\
6 & 12 & 1000060120 & $\mathrm{C}$ & 148.29 & 137898 & $5.25473 \mathrm{e}-06$ \\
7 & 14 & 1000070140 & $\mathrm{~N}$ & 0.0077127 & 6.62521 & $2.52459 \mathrm{e}-10$ \\
8 & 16 & 1000080160 & $\mathrm{O}$ & $4.24743 \mathrm{e}+08$ & $1.14681 \mathrm{e}+12$ & 43.7 \\
11 & 23 & 1000110230 & $\mathrm{Na}$ & $2.15773 \mathrm{e}+08$ & $5.82588 \mathrm{e}+11$ & 22.2 \\
13 & 27 & 1000130270 & $\mathrm{Al}$ & $4.76257 \mathrm{e}+07$ & $1.28589 \mathrm{e}+11$ & 4.9 \\
14 & 28 & 1000140280 & $\mathrm{Si}$ & $2.49792 \mathrm{e}+08$ & $6.74438 \mathrm{e}+11$ & 25.7 \\
15 & 31 & 1000150310 & $\mathrm{P}$ & 0.00434539 & 34.1548 & $1.3015 \mathrm{e}-09$ \\
16 & 32 & 1000160320 & $\mathrm{~S}$ & 0.00543174 & 42.6935 & $1.62687 \mathrm{e}-09$ \\
17 & 35 & 1000170350 & $\mathrm{Cl}$ & 23.4691 & 34968.9 & $1.33252 \mathrm{e}-06$ \\
19 & 39 & 1000190390 & $\mathrm{~K}$ & $1.45793 \mathrm{e}+07$ & $3.93641 \mathrm{e}+10$ & 1.5 \\
20 & 40 & 1000200400 & $\mathrm{Ca}$ & 5.02437 & 11556 & $4.40353 \mathrm{e}-07$ \\
22 & 48 & 1000220480 & $\mathrm{Ti}$ & 4.37551 & 6519.51 & $2.48431 \mathrm{e}-07$ \\
25 & 55 & 1000250550 & $\mathrm{Mn}$ & 0.111894 & 879.486 & $3.35135 \mathrm{e}-08$ \\
26 & 56 & 1000260560 & $\mathrm{Fe}$ & $1.94391 \mathrm{e}+07$ & $5.24855 \mathrm{e}+10$ & 2.0 \\
29 & 64 & 1000290640 & $\mathrm{Cu}$ & 0.021727 & 170.774 & $6.50748 \mathrm{e}-09$ \\
\hline
\end{tabular}

Table 3.2. The isotope list in the NOvA near detector GDML files.

NOvA uses wavelength shifting fibers to transport scintillation light, since the material for each cell are identical, so that we generate one template for scintillation light and then transport the photons through the fibers and APD readout system [18]. So that, after the hits were simulated in the detectors, we convert the simulated information (true information) to number of photons due to the origin and timing of each hit. We use a log-normal distribution for the APD response probability and then distribute the number of photon electrons with noise expansion to a function of time and then convert it into digital signals similar to the real data collected in the detectors. Figure 3.2 illustrate a GENIE based Monte Carlo event display in the NOvA near detector. 


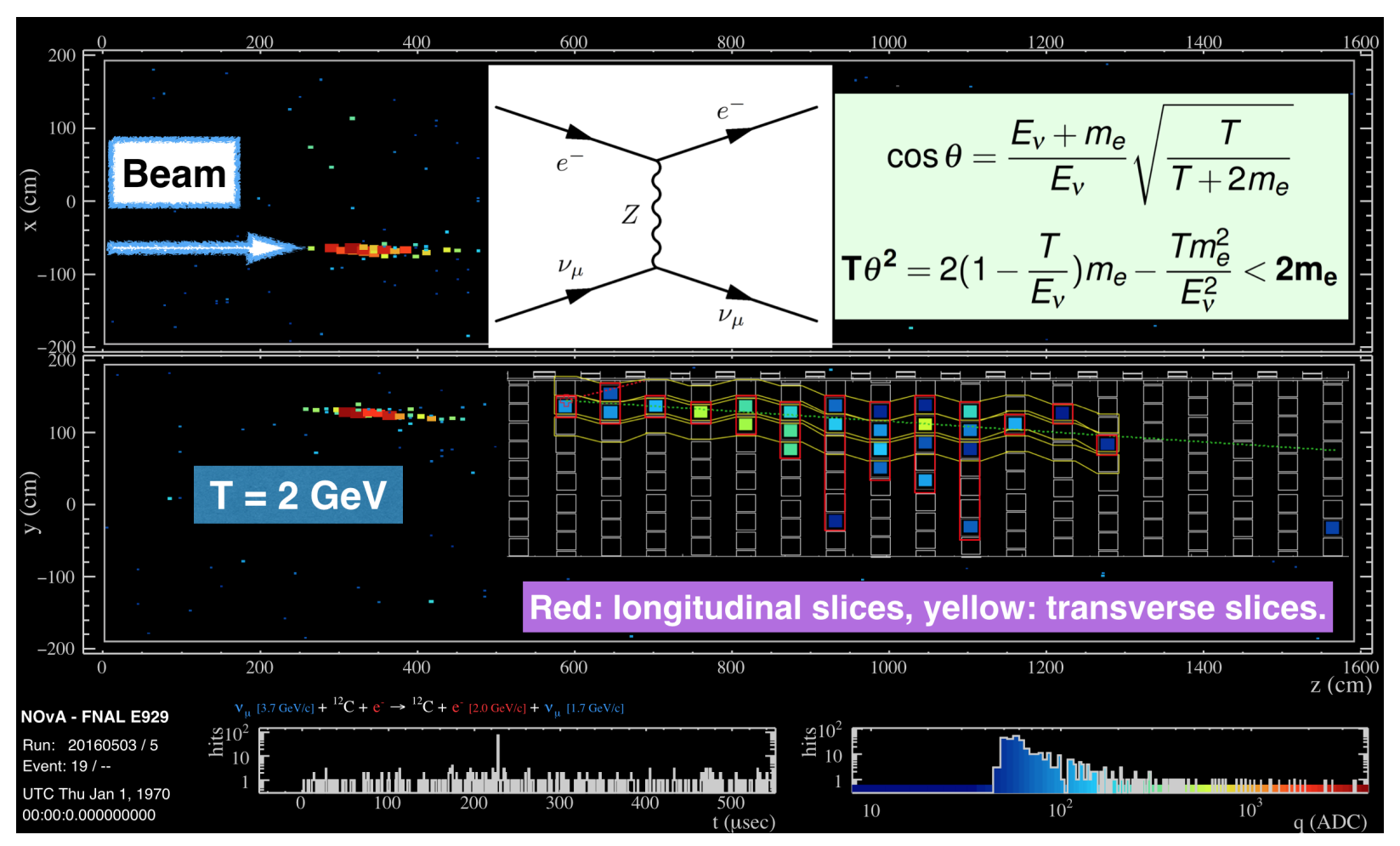

Figure 3.2. Event display for a $2 \mathrm{GeV}$ electron shower from a $\nu_{\mu}-e$ elastic scattering in NOvA near detector. [102]

Note that beside the cross-section measurement, $\nu_{e}$ appearance and $\nu_{\mu}$ disappearance analysis, NOvA is capable to search for other exotic topics. For example, we build the supernova trigger system on far detector, and the supernova simulation is similar as the oscillation simulation but switch the NuMI neutrino beam with the supernova neutrino flux files. Also, the HEPEVT standard text files can be applied to generate MC events directly in NOvA simulation software. This method helps a lot when we deal with the lightweight dark matter MC generation, there are too many hadronic process for the $\chi$ particle generation, the detector acceptance and also the total efficiency before the particle Identification. Which means, by using the HEPEVT method, one can calculate the final states of lightweight dark matter on electron elastic scattering by using Pythia or Fortran quickly and then get the daq root files with inputed systematic uncertainties. Then the showers will be generated by GEANT4 according to the true kinematic informations in the HEPEVT standard text files. The more convenient is that, it can be used in different experiment with the same 
true information of the lightweight dark matter signal files. So that the same exotic particle generation approach can be used in both NOvA and DUNE for example, helping analyst to compare the detector acceptance, total selection efficiency and unfolding methods. A more detailed description of the NOvA simulation chain can be found in Ref. [18]. 


\subsection{Reconstruction Chain}

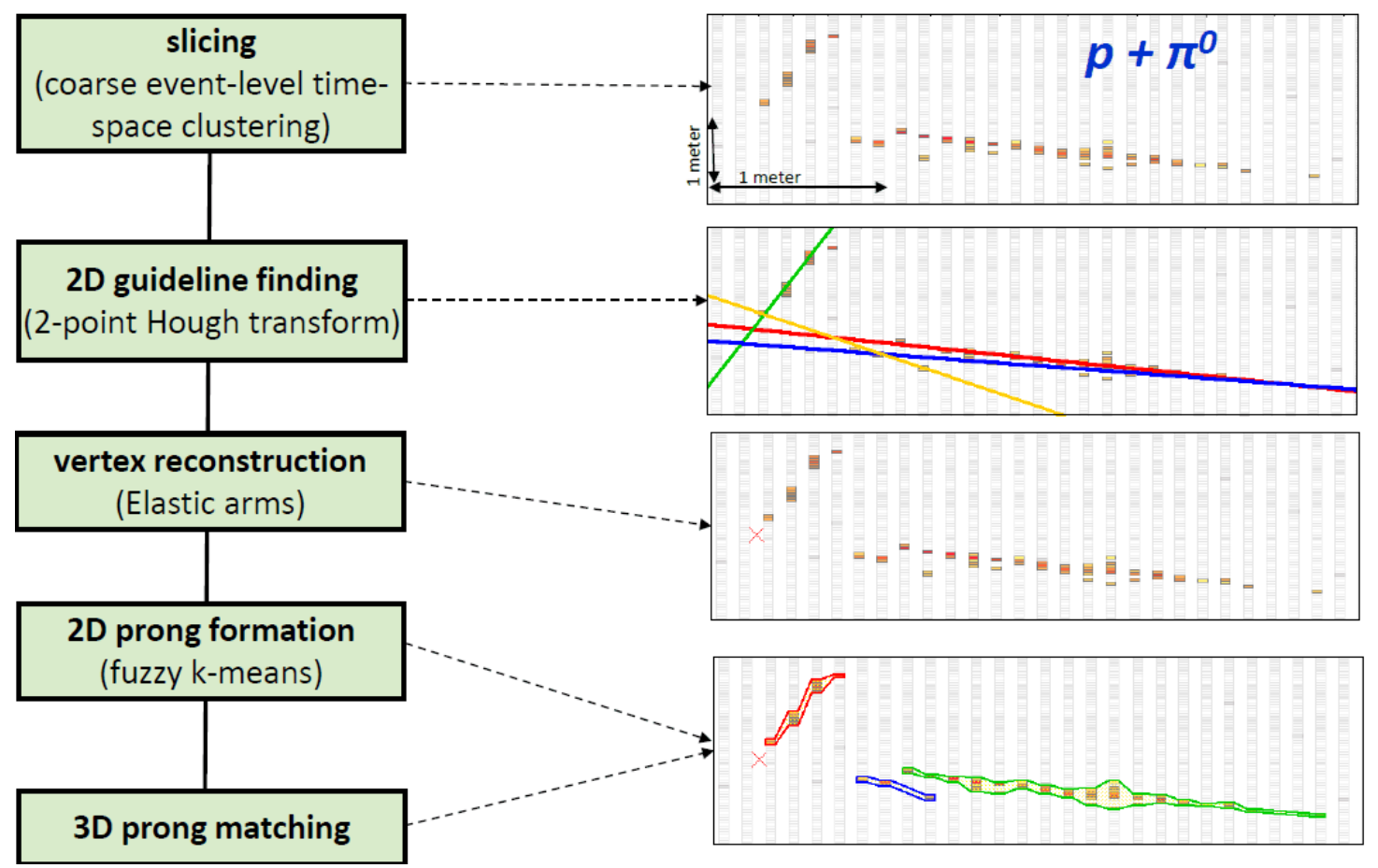

Figure 3.3. Reconstruction Chain for a general event in the NOvA experiment, the plot is originally from Ref. [76].

The reconstruction process we were using is identical to the $\nu_{e}$ analysis in NOvA. Firstly, we group the cell hits into one cluster by comparing timing and spacial information. We define a score function by

$$
\epsilon=\left(\frac{\Delta T-\Delta r / c}{T_{\text {res }}}\right)^{2}+\left(\frac{\Delta r}{D_{\text {pen }}}\right)^{2} .
$$

For any hit in the same event, if another nearest hit has the score $\epsilon$ is less than a threshold value $\epsilon_{0}$, then we define them as neighbor and then continue to loop all the hits until no more neighbor can be found in that event [22]. By this way, we separate event interactions into clusters. Then the energy of shower is calculate by converting the visual energy of that shower cluster to reconstructed energy. 
Next step, we use a pattern recognition method to draw feature guidelines for different tracks that characterize the event with a multi-hough transform. Then these guidelines were used as arms in the Elastic Arms method to find the optimum vertex candidate.

$$
E=\sum_{i=1}^{N} \sum_{a=1}^{M} V_{i a} M_{i a}+\lambda \sum_{i=1}^{N}\left(\sum_{a=1}^{M} V_{i a}-1\right)^{2}+\frac{2}{\lambda_{v}} \sum_{a=1}^{M} D_{a}
$$

The energy loss function is defined in Eq. 3.2, where $M_{i a}$ is the distance between arm a and hit i, $D_{a}$ measures the vertex and the first hit in arm a. $V_{i a}$ represented the association between hit $\mathrm{i}$ and arm a, $\lambda$ is used for controlling the strength. The vertex is found by minimizing this energy loss function.

Once the vertex is determined, we use the fuzzy-K algoritm to determine the core of each shower and thus reconstruct the angular information $\theta$ from the vertex point. The 0 radian is the forward $\mathrm{Z}$ direction in each detector. The distance for each cell hit $\mathrm{j}$ to current cluster center $\mathrm{i}$ is given by

$$
d_{i j}=\left(\frac{\theta_{j}-\bar{\theta}_{i}}{\sigma_{j}}\right)^{2} .
$$

Then a degree of membership for each hit $\mathrm{j}$ is computed by

$$
U_{i j}=e^{\frac{m \sqrt{c} d_{i j}}{\beta}} .
$$

Here $\beta$ is a term for normalization, $\mathrm{c}$ is the number of prong centers, $\mathrm{m}$ is fuzziness factor that represented the membership of one hit in multiple prongs. Hit $\mathrm{j}$ with membership degree higher than $1 \%$ is associated with prong i. Then we update the prong center angle with a higher precision by

$$
\theta_{i}^{\prime}=\theta_{i}+\frac{\sum_{j=1}^{n} U_{i j} / \sigma_{j}^{2} \times\left(\theta_{j}-\bar{\theta}_{i}\right)}{\sum_{j=1}^{n} U_{i j} / \sigma_{j}^{2}}
$$

Then, after we figure out the angular information for both 2-D view, we match them together to get a 3-D angular information. More details can be found in Ref. [76]. 


\subsection{Particle Identification}

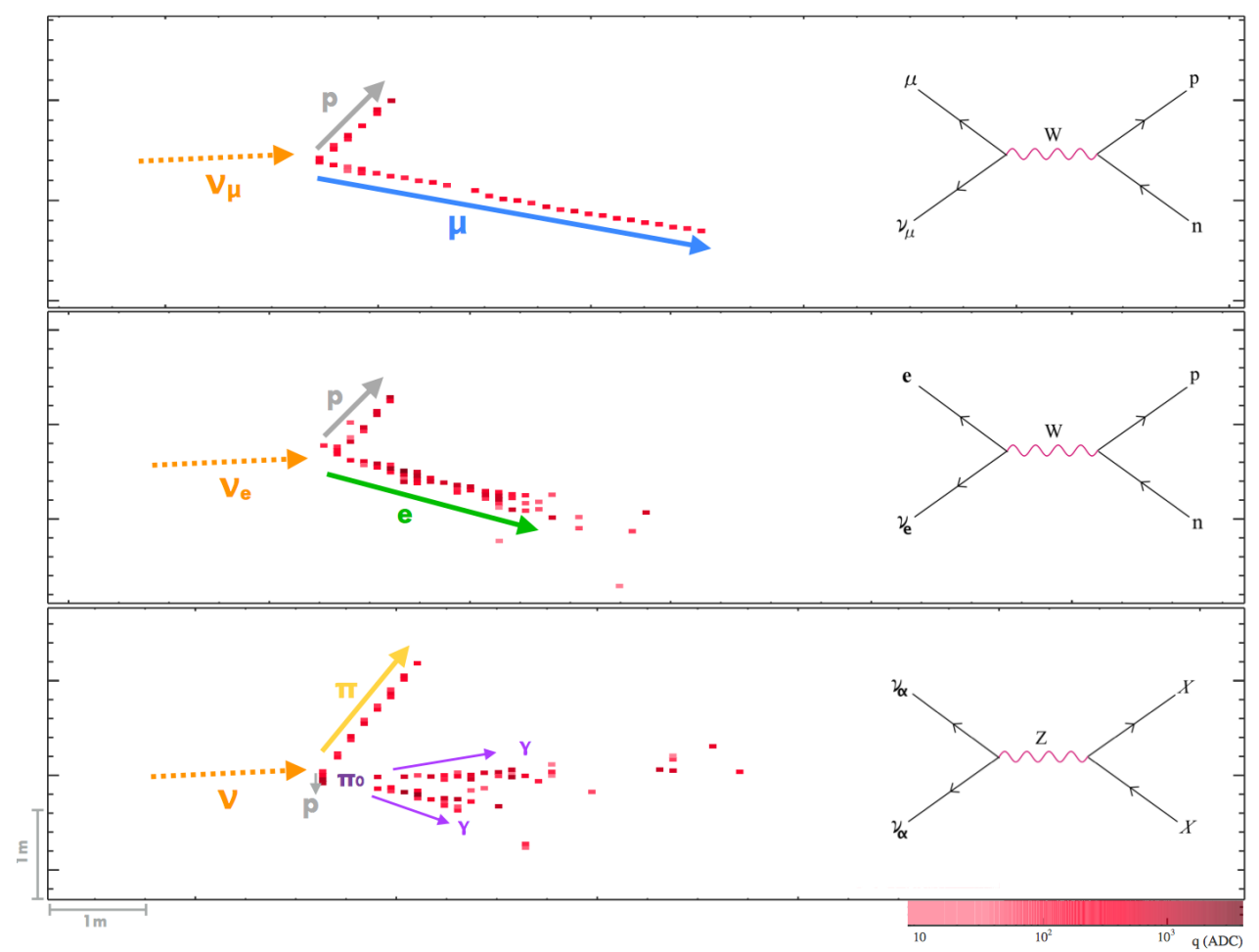

Figure 3.4. The event displays for $\nu_{\mu}-N$ charge current scattering, $\nu_{e}-N$ charge current scattering and $\nu$ - $N$ neutral current scattering.

Figure 3.4 shows three types of major interactions that occur in the NOvA detector. We select $\nu_{e}-N$ charge current scattering for $\nu_{e}$ appearance analysis and $\nu_{\mu}-N$ charge current events for $\nu_{\mu}$ disappearance analysis. $\nu$ - $N$ neutral current scattering has been used in search for sterile involved oscillations. Unfortunately they become the background of $\nu$-e elastic scattering and we have to remove them. The strategy of the analysis regarding the $\nu$-e elastic scattering and the muon neutrino magnetic moment are the followings. 


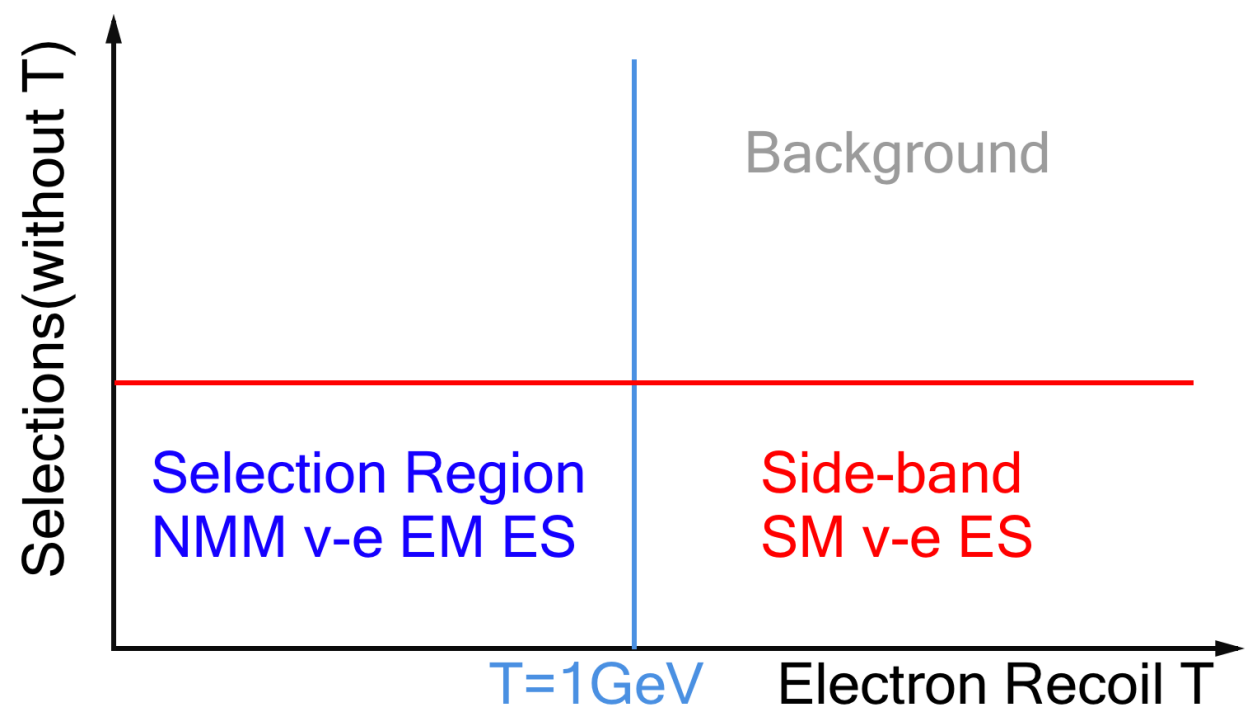

Figure 3.5. The diagram shows the strategy of this analysis, the $\nu_{\mu}-N$ charge current scattering, $\nu_{e}-N$ charge current scattering and $\nu-N$ neutral current scattering are the background to be removed.

The original fraction of $\nu$-e elastic scattering (ES) events is quiet tiny, roughly 1 ES event in 10000 total events inclusively. Thus, in order to estimate the upper limit of $\nu_{\mu}$-e magnetic scattering, we have to confirm that the selection for $\nu$-e weak scattering in Standard Model is valid.

We firstly apply the following selection criteria to determine the fiducial volume and the single electromagnetic shower in the NOvA near detector. We choose $-170 \mathrm{~cm}<\mathrm{X}<$ $170 \mathrm{~cm},-170 \mathrm{~cm}<\mathrm{Y}<170 \mathrm{~cm}$ and $200 \mathrm{~cm}<\mathrm{Z}<1200 \mathrm{~cm}$ as the fiducial volume, where the $X, Y$ and $Z$ are the positions of hits in the detector. We also choose the length of the track less than $800 \mathrm{~cm}$, the number of showers crossed plane less than 120 and the number of total hits less than 600 in a single shower to save the computing time. Then we select the events with single non-muon related shower. We choose the gap between the starting point of the shower and the reconstructed vertex less than $20 \mathrm{~cm}$, while there is no energy deposited around the vertex within eight planes. We also only select the event that the leading shower energy takes more than 90 percents of the total energy stored in that event. 
Then the next step is that we look into the region for $T>1 \mathrm{GeV}$, which is $\nu$-e weak scattering dominant and then start training two particle identification to reject the $\nu_{\mu}-N$ charge current events and the neutral current $\pi^{0}$ background. After the PIDs are determined, then we optimize the selection of the kinematic information and look into the data and simulation comparison for $\nu$-e weak scattering to check the neutrino flux and overall selection validation.

The last step is to determine sensitive region for muon neutrino magnetic moment involved $\nu_{\mu}$-e magnetic scattering. We freeze those selections except the T region and then look into the signal $\mathrm{T}$ region for the search for the muon neutrino magnetic moment (Fig. 3.5).

\subsubsection{Log Likelihood Identification}

In this subsection, we use a multilayer perceptron neural network for particle identifications training, a method similar to that used in the first results of $\nu_{e}$ appearance analysis in the NOvA experiment $[5,33]$ (LID). The log-likelihood of the observed $d E / d x$ matching one of multiple particle hypotheses (e to $\pi^{0}, \gamma, \pi^{ \pm}, p, n$ and $\mu$ ) for both longitudinal and transverse shower direction is used to identify an elastically scattered electron in an inclusive dataset and remove $\nu_{\mu}$ charge current events. 


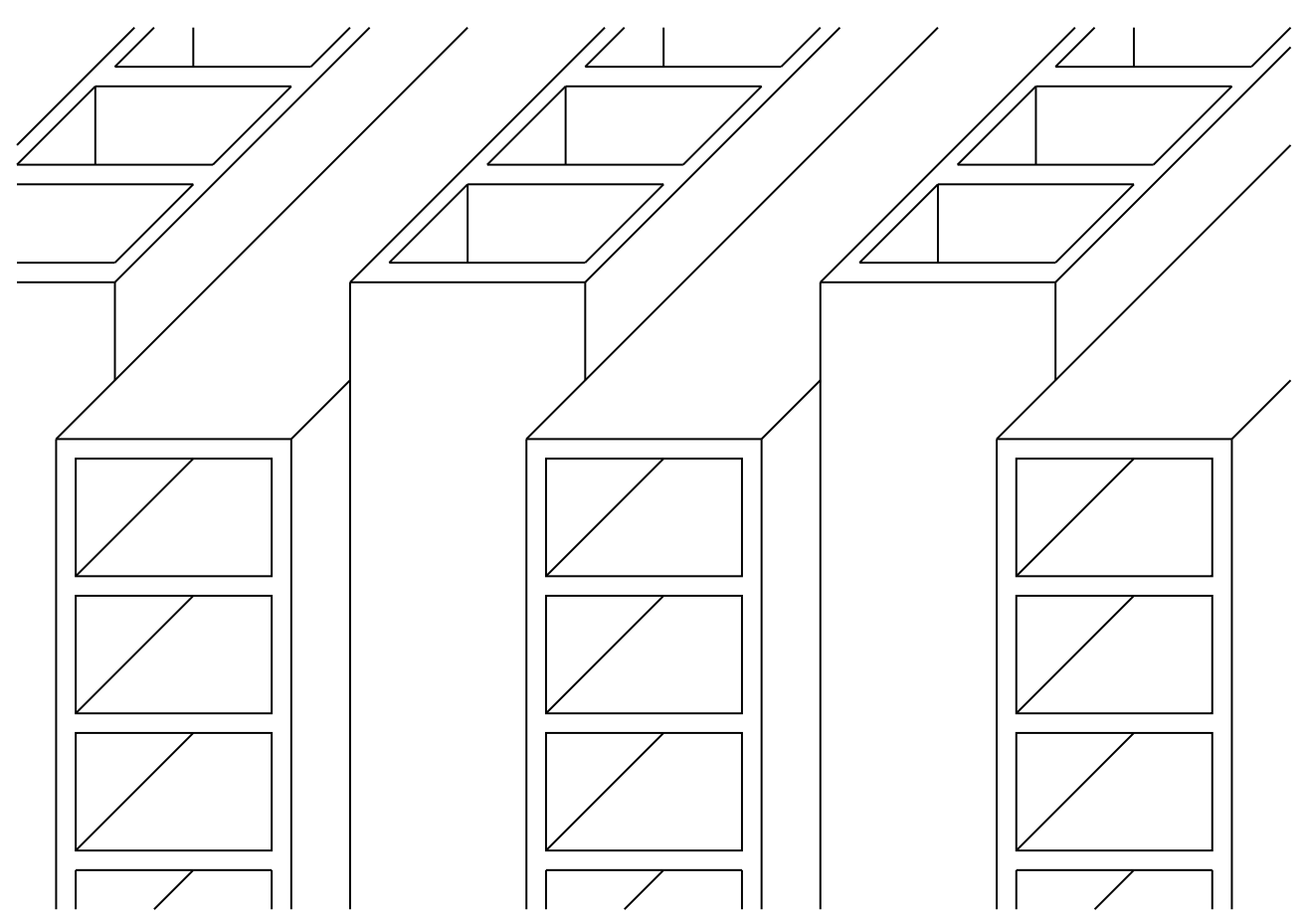

Figure 3.6. The structure of the NOvA detectors with vertical and horizontal alternating orientations, this diagram is originally from Ref. [21].

Although NOvA has already developed the CVN PID by deep learning the visual event display image, the fundamental identification process is still a string by string process since the readout is still from each APD. The feature of high energy charged particle can be described by $d E / d x$ in the detector. For the case in NOvA near detector, recall the structure of the NOvA detector in Fig. 3.6, the fundamental energy loss can only be calculated for each cell and each plane. In other words, we can compute the longitudinal energy loss by comparing $d E / d x$ in the contiguous plane as shown in Fig. 3.7 by using the total deposited energy in that plane divided by the path length.

To be more intuitive, let's consider the case that there are two isolated islands, A and B in a virtual world. The only thing we know is that their shapes of hands are different for those two islands. Now the question becomes that by looking at or scanning one person's hands, how do we know whether he is coming from island A or island B. In order to tell the difference, one has to known the general distributions for the width and length of each 
finger for the people living in those islands. Through the comparison of ten fingers width and length, matching each of those variable to the overall distribution of fingers fo the inhabitants in those two islands, we are able to make an Identification based on the sum of those likelihoods.

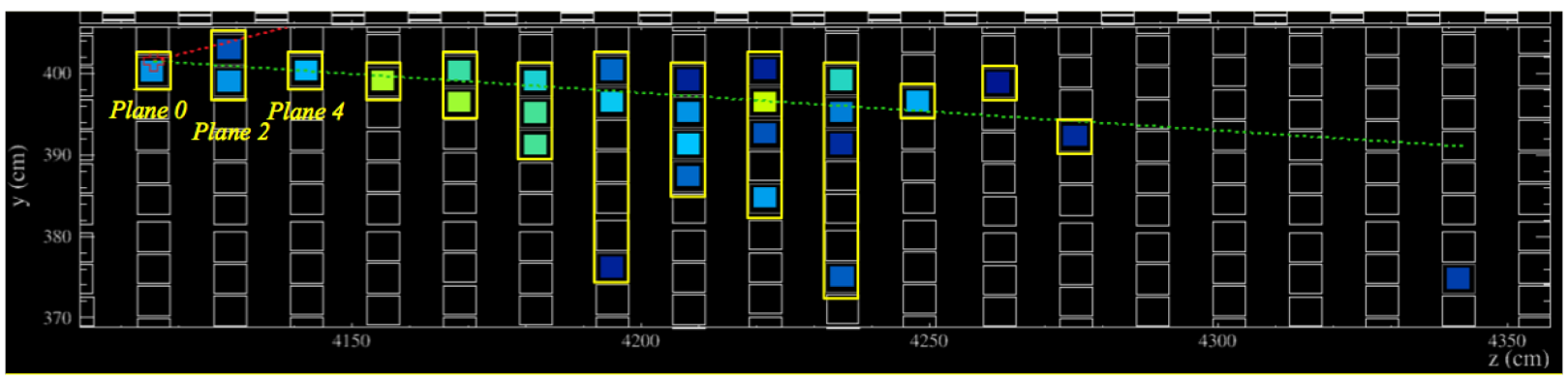

Figure 3.7. The longitudinal component of $d E / d x$.

Similarly in the NOvA experiment, the distribution difference of particle energy loss become the variables to identify the particle. Every type of particle has its own most probable Landau distribution for the energy loss. In practice, the $d E / d x$ will behave differently for different longitudinal plane. Take the electron and muon comparison for example, the energy depositions look alike in the second plane (plane index $=1$ ), but differently in the 11th plane (plane index $=10)$ since the muon keeps minimum ionizing deposition (Fig. 3.8).
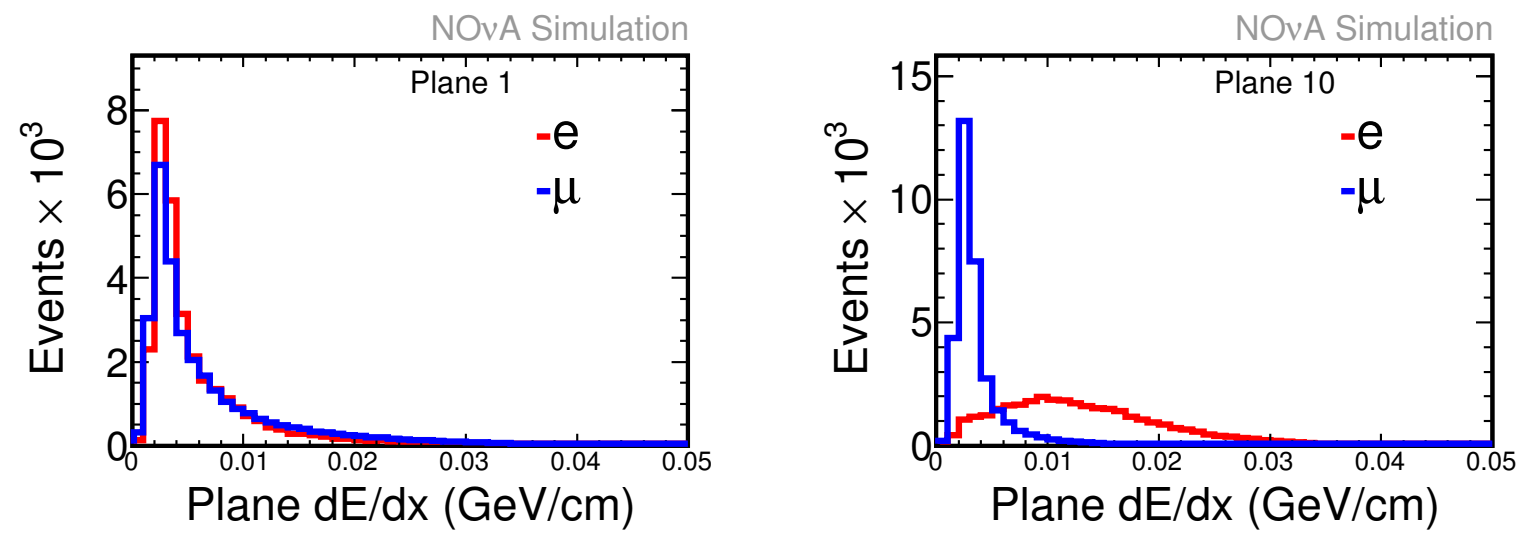

Figure 3.8. Longitudinal $d E / d x$ for electrons (red) and muons (blue). 
Then, since the $d E / d x$ distribution for electrons and muons are different, then if we found a particle with the $d E / d x \sim 0.01 \mathrm{GeV} / \mathrm{cm}$ in the 11th plane, so that it is more likely to be an electron than muon.

Generally speaking, we estimate the probability of an incident particle to be one of the known MC hypothesis in the $i^{\text {th }}$ plane by

$$
p_{i}=\frac{\text { that } d E / d x \text { bin area }}{\text { total histogram area }} .
$$

And its value can be represented by the ratio of entries in

$$
p_{i}=\frac{N_{\text {single bin }}}{N_{\text {total }}} .
$$

Ideally, Eq. 3.7 represents the possibility of matching a certain particle by using an observed $d E / d x$, and it might rise at least two kind of risks in some realistic cases.

The first risk is that in each plane whenever what kind of MC hypothesis histogram library we are using, the possibility of $d E / d x$ always decrease dramatically when the $d E / d x$ goes larger. Which means, by using the $p_{i}$ we defined in Eq. 3.7, when $d E / d x$ goes larger, the $p_{i}$ behaves ultra tiny that even below the machine precision of double float. And the second following risk is that it makes the difference between different $\mathrm{MC}$ particle hypothesis vague or less than the machine precision.

Following the philosophy of Occam's Razor, we multiply a scale factor for each of the $p_{i}$ as shown in

$$
p_{i k}=\frac{N_{k} \times k}{N_{\text {total }}} .
$$

Which means, suppose the incident known particle cause $d E / d x$ locate in the $k^{t h}$ bin in the $i^{\text {th }}$ plane, the probability is the nominal ratio times the bin number $\mathrm{k}$. So that when $d E / d x$ goes larger, the probability is scaled up by a factor of $\mathrm{k}$ artificially, which proportional to $d E / d x$, so that make sure the probability is larger enough not only to simplify the rest of 
calculation but also to distinguish different MC hypothesis.

Then taking the logarithm of it so that convert the calculation to simply plus or minus

$$
L L_{i}=\ln p_{i}
$$

Then we sum up the log-likelihoods from the first plane to the last plane and then divided by the number of planes

$$
L L_{L}=\frac{\sum_{i=1}^{\text {last }} L L_{i}}{N_{\text {planes }}} .
$$

By now, we have defined the longitudinal log-likelihood of a certain MC hypothesis.

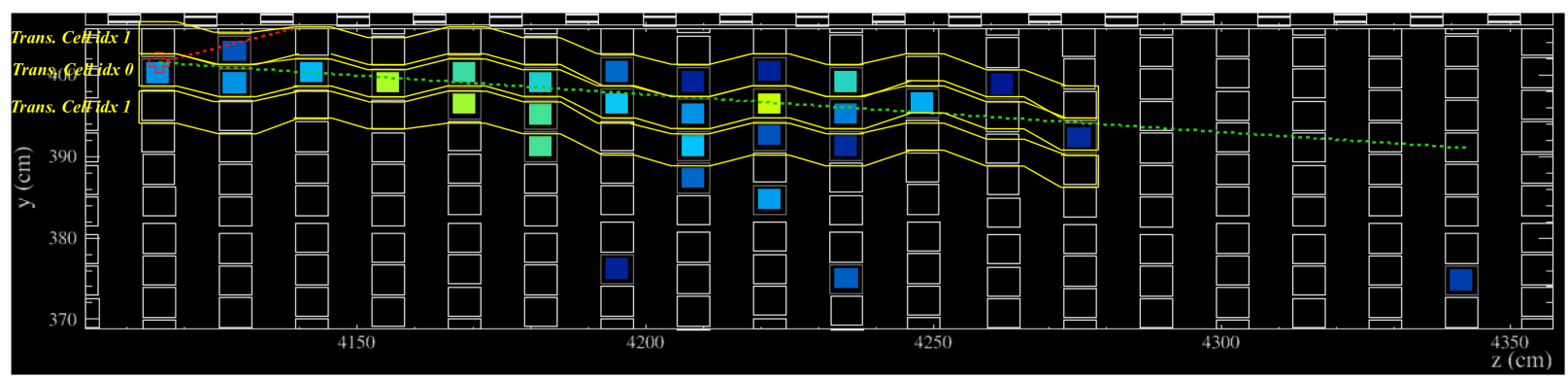

Figure 3.9. The transverse cell definition for $d E / d x$.

For transverse cells, we firstly draw a straight line from the start point of a shower to the end point of that shower. In each plane, the cell with the intersection point of that plane and the straight line locates in is decided to be index 0 of transverse cell. Then for both directions, the cell next to index 0 is index 1, and up to 20 indexes. The transverse energy deposition is taking the average of both sides and then divided by the total path length, we get the transverse $d E / d x$ corresponding to a certain cell index. Similarly, we get the transverse log-likelihoods function by

$$
L L_{T}=\frac{\sum L L_{i}}{N_{\text {transverse cells }}} .
$$

This method avoid the risks of machine precision and the tiny difference. However, it 
also caused another two problems. For the obvious one is that the probabilities are not going to be normalized. And the other related problem is that if we put these variables as machine learning inputs, somehow for the case that $N_{k 1} \times k 1=N_{k 2} \times k 2$ may cause confusions in following analysis. So in order to avoid these problems, we compare the particles we want to select to all the other particles that we want to remove to build the log-likelihood functions

$$
L L_{i}(e / x)=\ln p_{i}(e)-\ln p_{i}(x) .
$$

Here $x$ is one of the six following particles, photon, proton, neutron, muon, charged pion and neutral pion. In this way, although we have multiply a scale factor of $\mathrm{k}$, it doesn't matter for the final results and also the input variables are not correlated. As a result, the following 12 variables in Fig. 3.10 to Fig. 3.15 are decided to be the input variables for particle identification training.
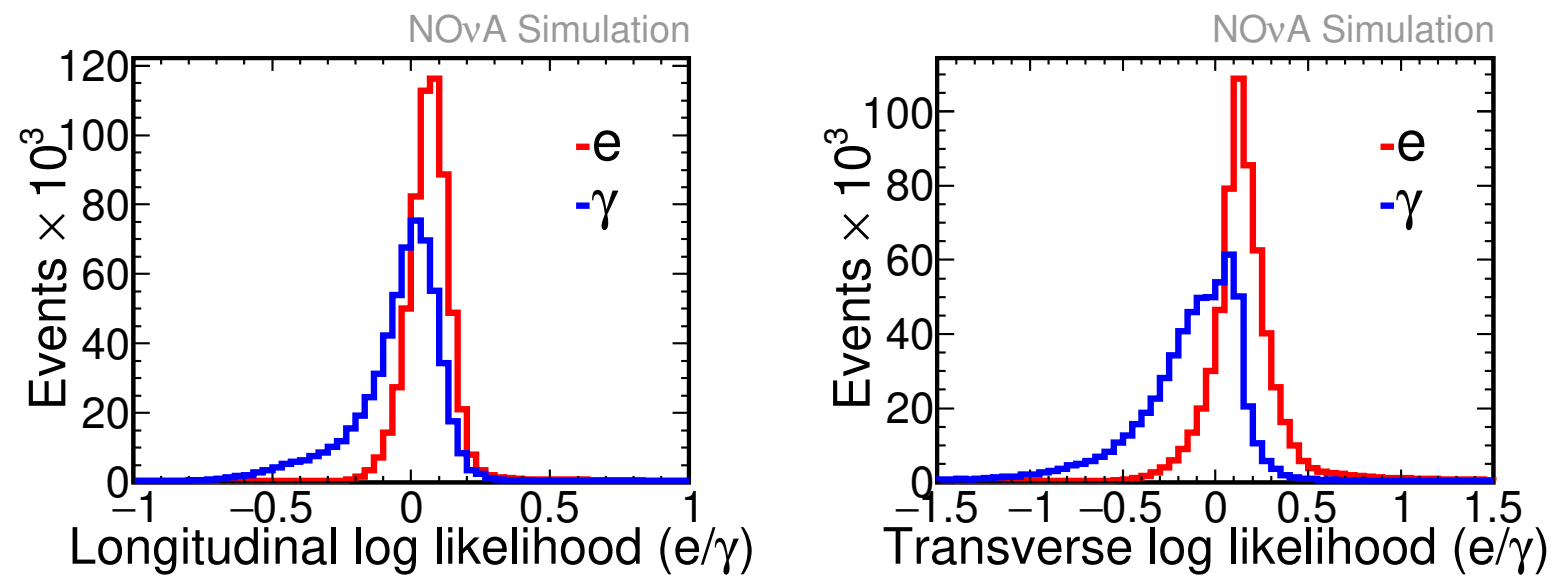

Figure 3.10. Comparing log likelihood of electron to $\gamma$, similar methods as described in Ref. [87]. 

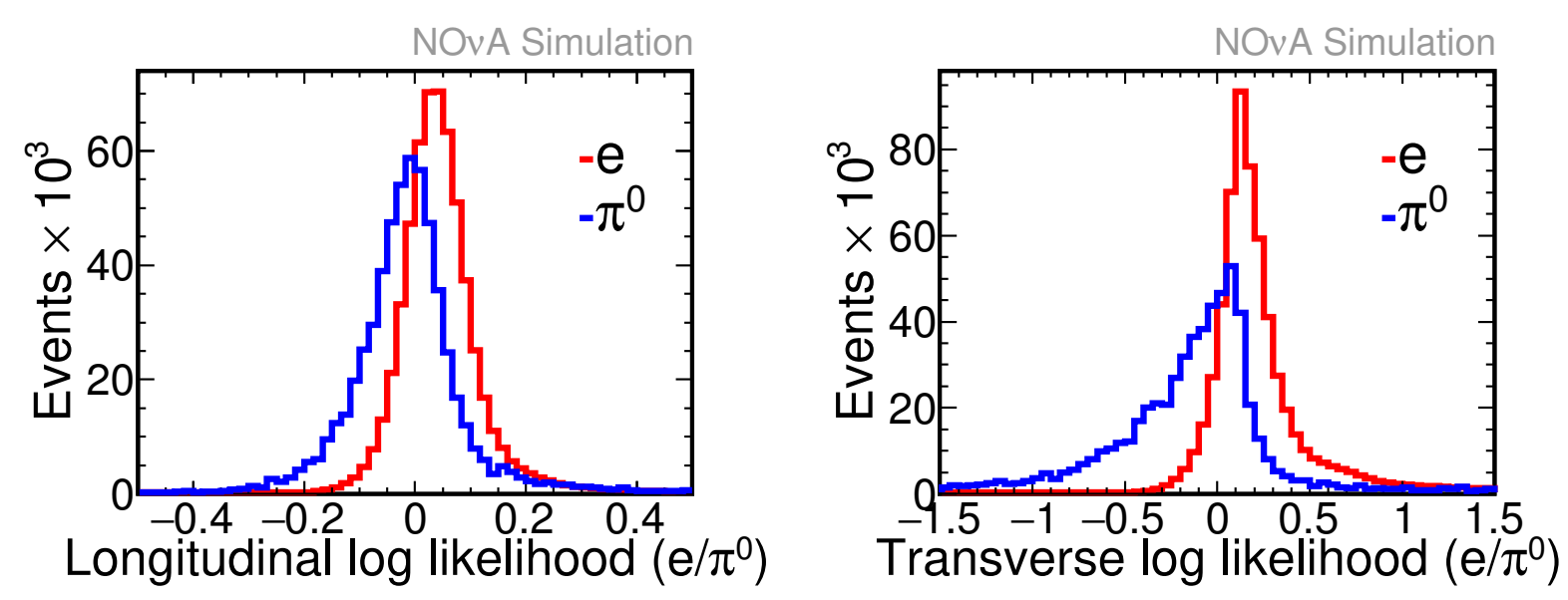

Figure 3.11. Comparing log likelihood of electron to neutral pion, similar methods as described in Ref. [87].
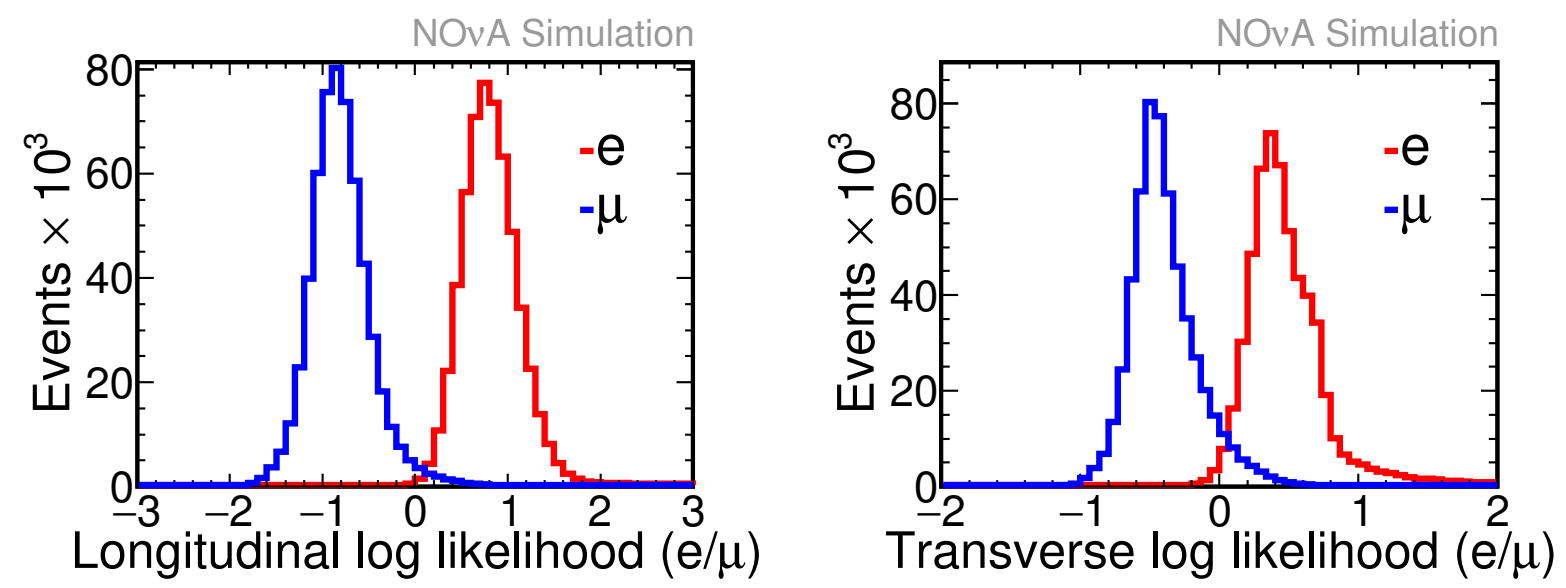

Figure 3.12. Comparing log likelihood of electron to muon, similar methods as described in Ref. [87]. 

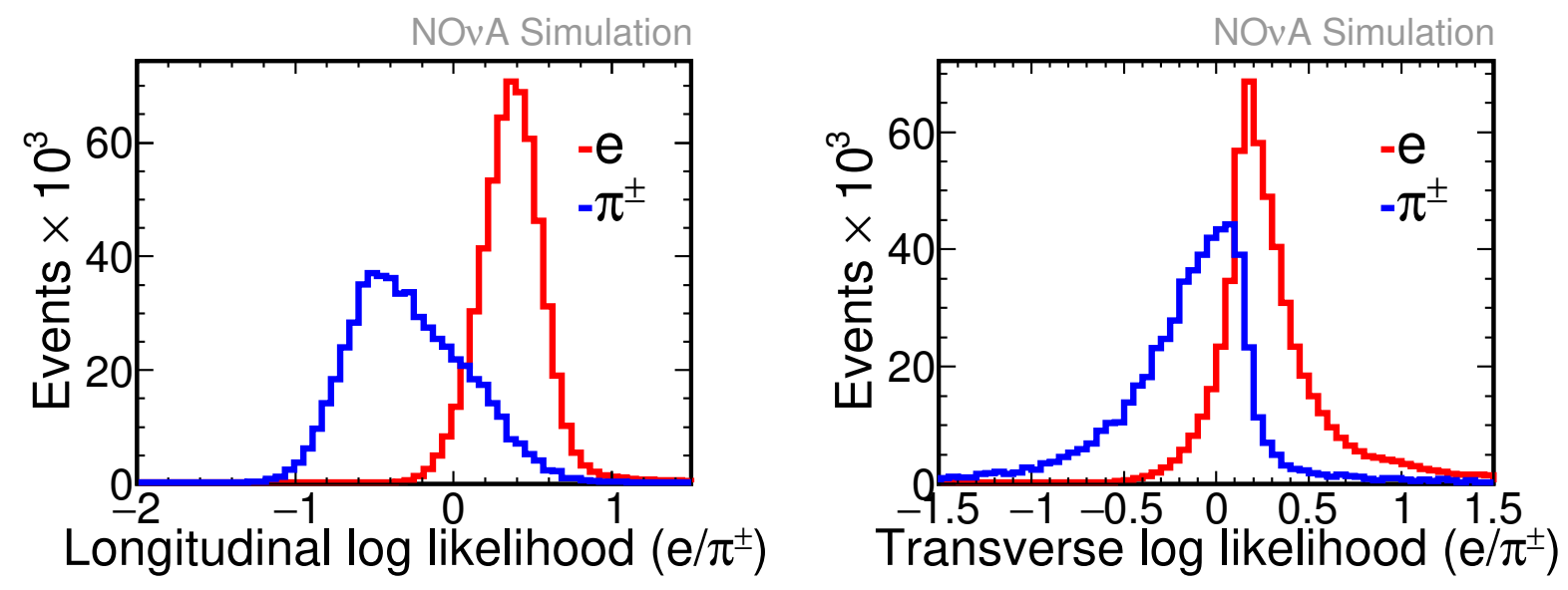

Figure 3.13. Comparing log likelihood of electron to charged pions, similar methods as described in Ref. [87].
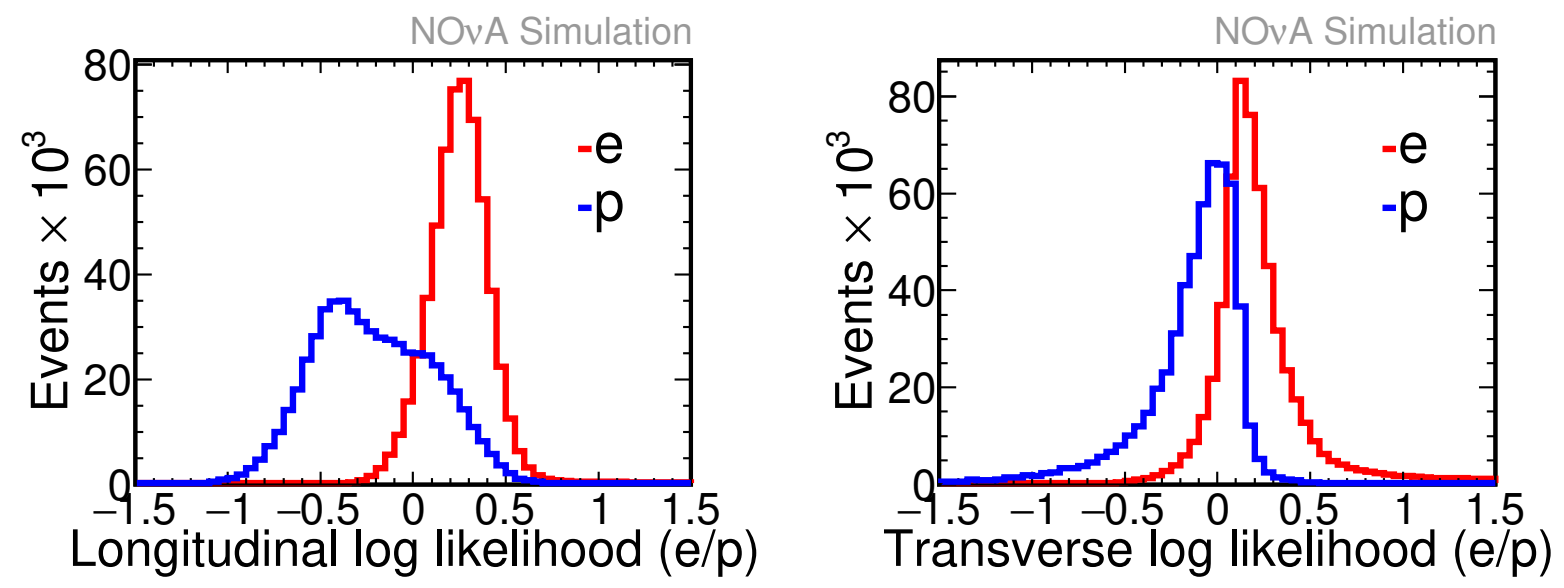

Figure 3.14. Comparing log likelihood of electron to proton, similar methods as described in Ref. [87]. 

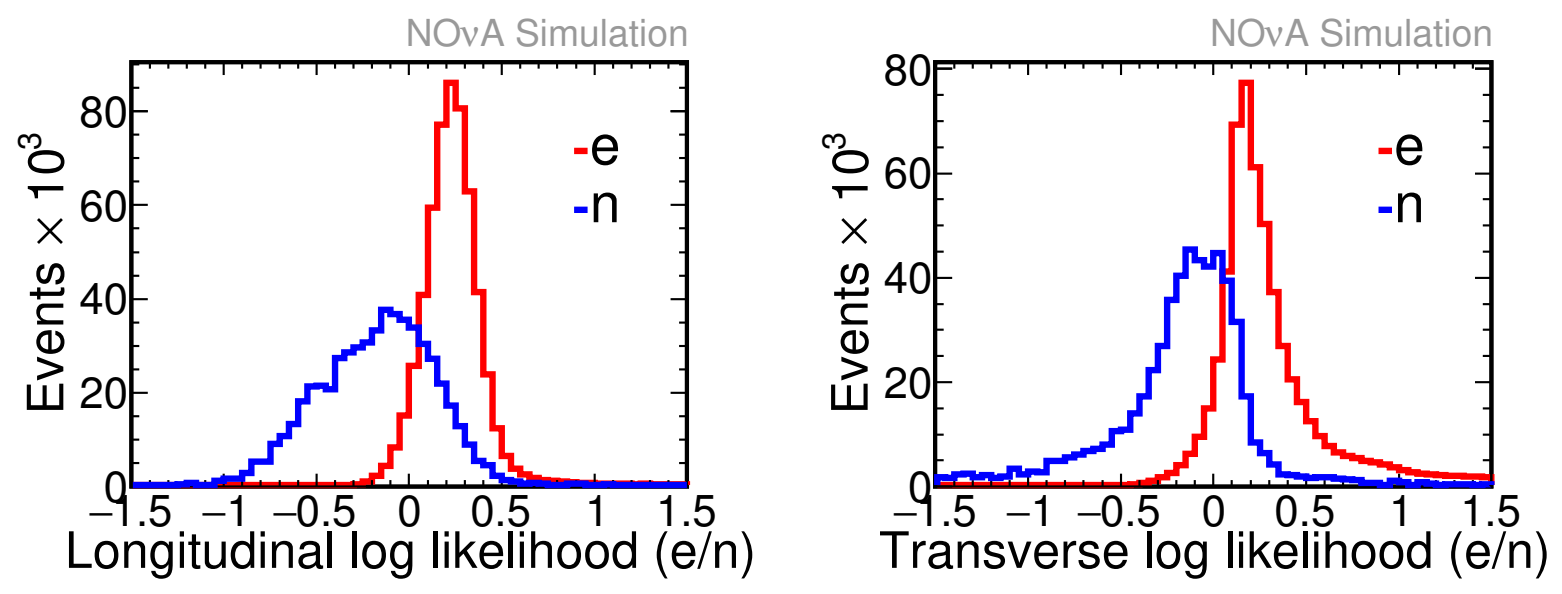

Figure 3.15. Comparing log likelihood of electron to neutron, similar methods as described in Ref. [87].

The multilayer perceptron neural network package provides a framework that made of neutrons in multiple layers. This software has been implemented in ROOT as a function called TMultiLayerPerceptron. It weighted different links from all the input variables through all the possible neurons in each layers to the output PID. The software will generate a text files with all the weighting numbers that calculated from the known MC training samples. So that each time we proceed an unknown shower, it load these weighting numbers from the text files and get the PID value.

For the first neutrino-electron elastic scattering PID to remove $\nu_{\mu}-N$ charge current events, 12 variables as mentioned earlier are used to form the input of a multivariate neural network. These inputs $\left(L L_{L}(e)-L L_{L}(\gamma), L L_{T}(e)-L L_{T}(\gamma)\right.$, etc) consist of 12 different template distribution between $\mathrm{dE} / \mathrm{dx}$ based longitudinal and transverse log-likelihoods for electron and the six other-particle hypotheses $\left(\gamma, \mu, \pi^{0}, p, n\right.$ and charged $\left.\pi\right)$ applied to the most energetic single EM shower.

The method of building the multilayer is following the semi-empirical relationship for a set of input variables in $j$ dimensions.

$$
2(j-1): j: \frac{j}{2}
$$


The likelihoods for $\gamma, \mu, \pi^{0}, \pi^{ \pm}, p, n$ are the same as the LID algorithm in NOvA first analysis of $\nu_{e}$ appearance, while longitudinal and transverse likelihoods for electrons are created with a single electron MC sample, which is exactly the sample that the $\nu$-e elastic scattering process calls in the GENIE generator.

Choosing the $\nu$-e elastic scattering as the signal and the $\nu_{\mu}-N$ charge current inclusive interaction events as the background, using three hidden layers as 22:12:6, we are able to produce the $\nu$-e to charge current events PID as shown in Fig. 3.16. Since the $\nu_{\mu}-N$ charge current events are thousands times more than the $\nu$-e elastic scattering events, we have to make some pre-selections so that the Fig. 3.16 looks more readable.

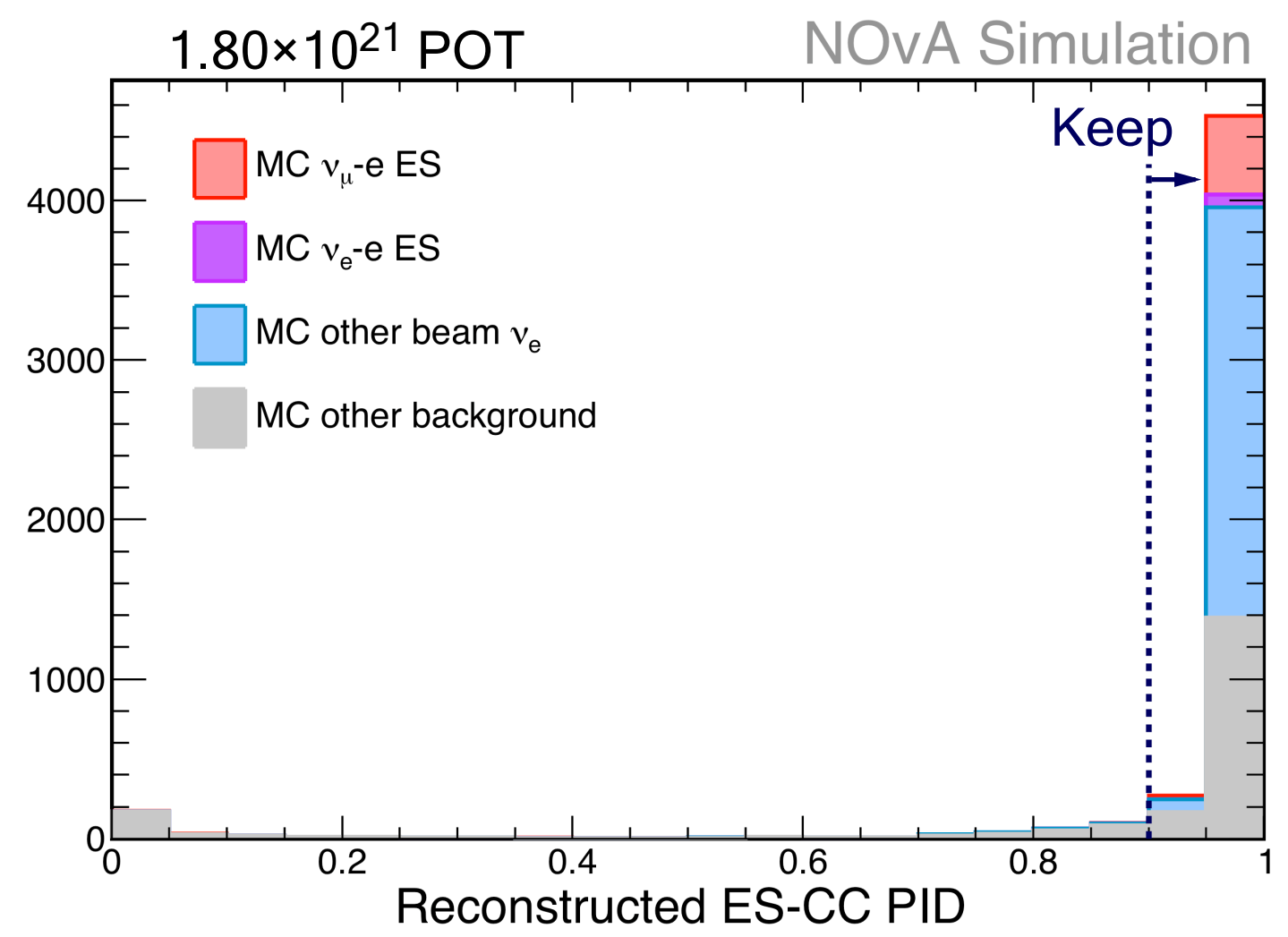

Figure 3.16. Performance for ES-CC PID. This is a plot after pre-selections. The performance of this PID is originally quite polarized, most of the $\nu_{\mu}$ charge current events were taged with ES-CC PID $<0.3$, so that they were removed in pre-selections.

Recall that the original ES to total events ratio is about $\frac{1}{10000}$, and after this ES-CC PID $>0.9$ selection, we get $600 \nu_{\mu}-e$ elastic scattering events amongst 6000 total events in 
an inclusive dataset for $1.8 \times 10^{21}$ POT. So that the purity of $\nu_{\mu}$-e elastic scattering events enhanced from $\frac{1}{10000}$ to roughly $\frac{1}{10}$.

\subsubsection{Neutral Current Pions as Major Background}
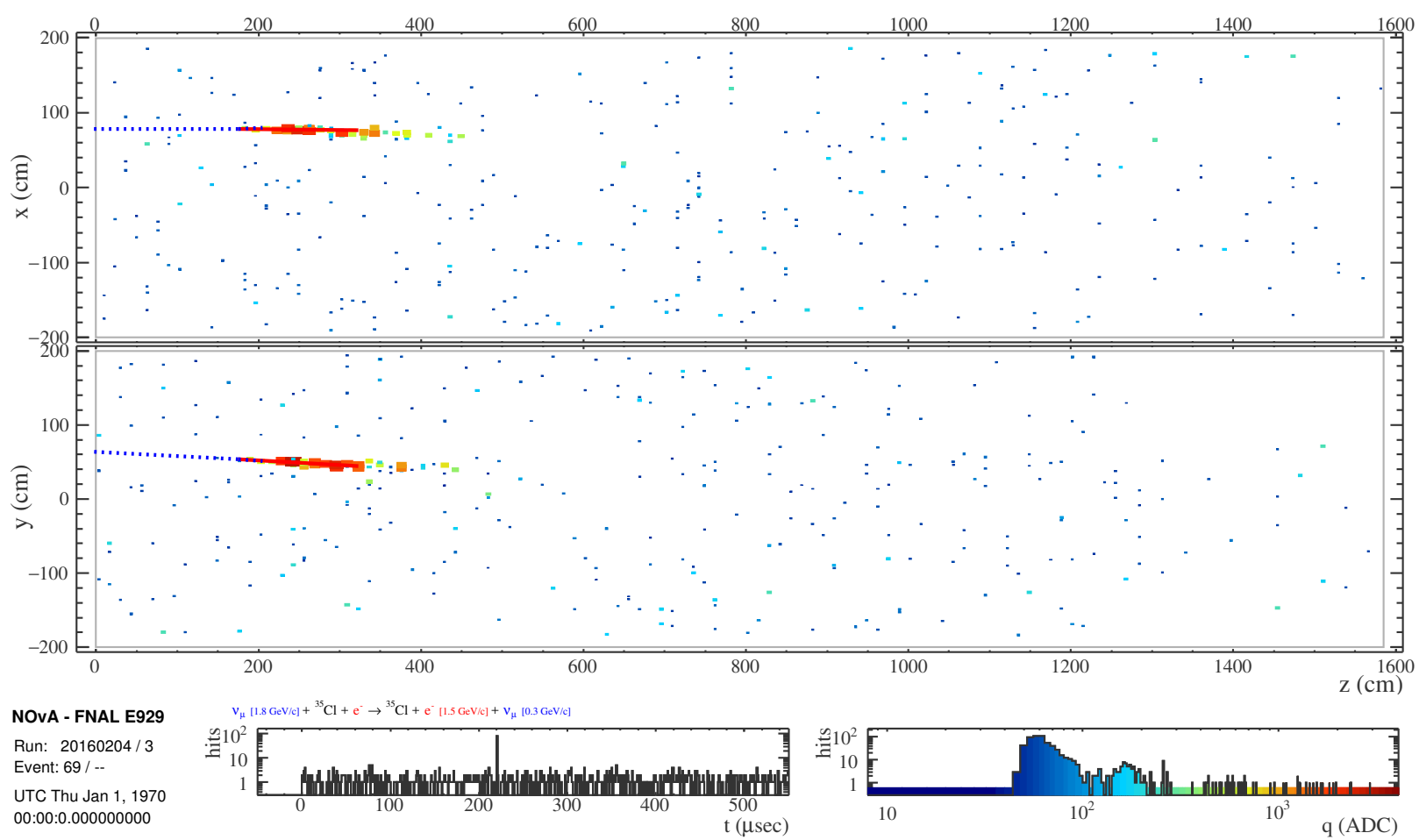

Figure 3.17. A typical example of $\nu_{\mu}$ on electron elastic scattering resulted electromagnetic shower.

The major background after the first neutrino-electron elastic scattering PID selection shown in last subsection are neutral current $\pi^{0} s$ with two daughter photons merged to one single shower or partially reconstructed in the detector, we introduce an ES-NC $\pi^{0}$ PID to distinguish electrons (Fig. 3.17) from these neutral current $\pi^{0} \mathrm{~s}$ (Fig. 3.18 and 3.19) in this subsection. 

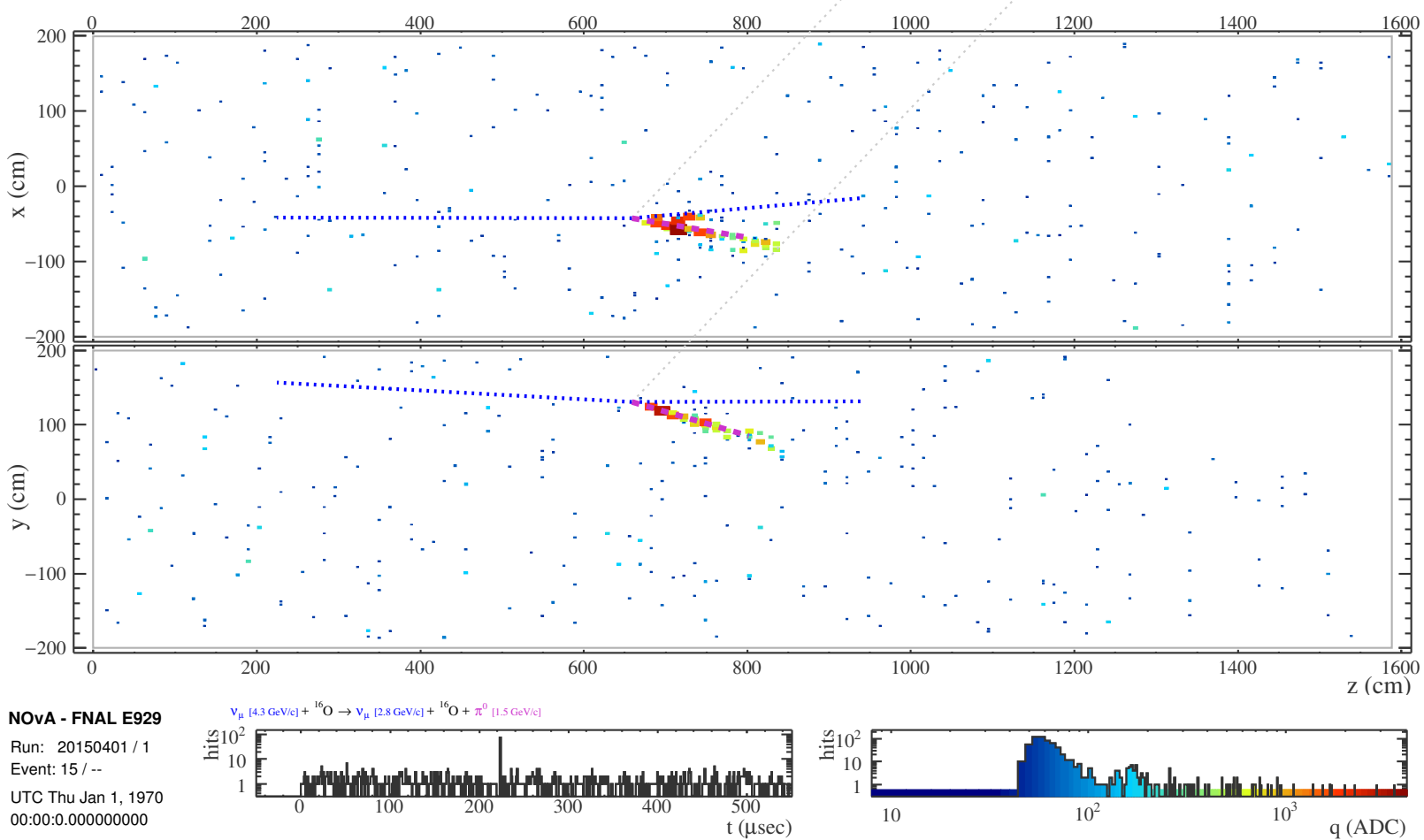

Figure 3.18. An example of pion induced electromagnetic shower, the shower property is very similar as the scattered electron shower. 


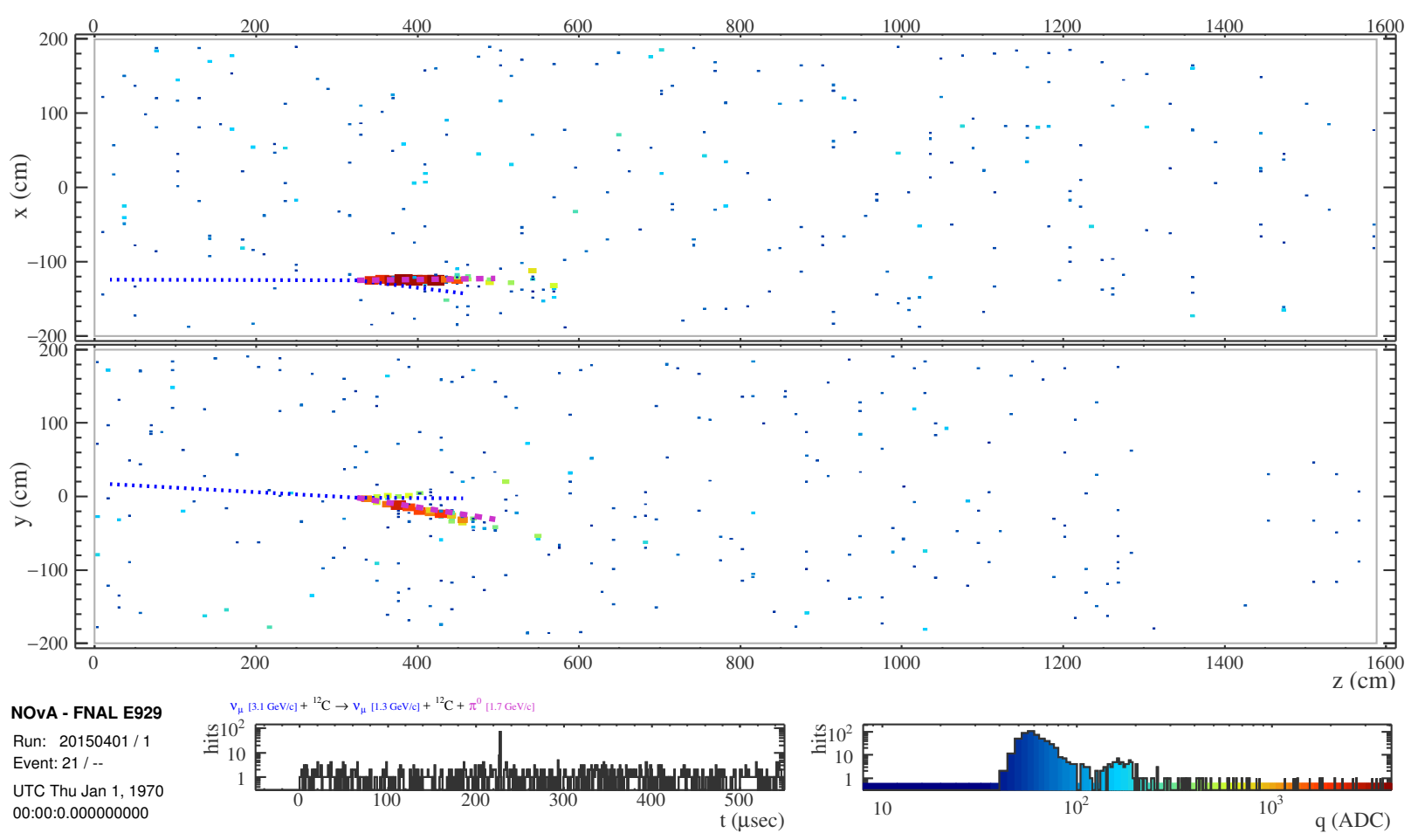

Figure 3.19. Another example of neutral current $\pi^{0}$ background, the shower property is also close to the scattered electron shower.

Despite of the angular distribution, which will be applied after the particle identification, we recognize that these electromagnetic showers from electrons and pions are quite similar as each other. The same challenges have been met and solved previously in CHARM experiment (Fig. 3.20), the energy deposition in the planes following the shower vertex are used to differentiate the electrons and neutral pions [50]. 


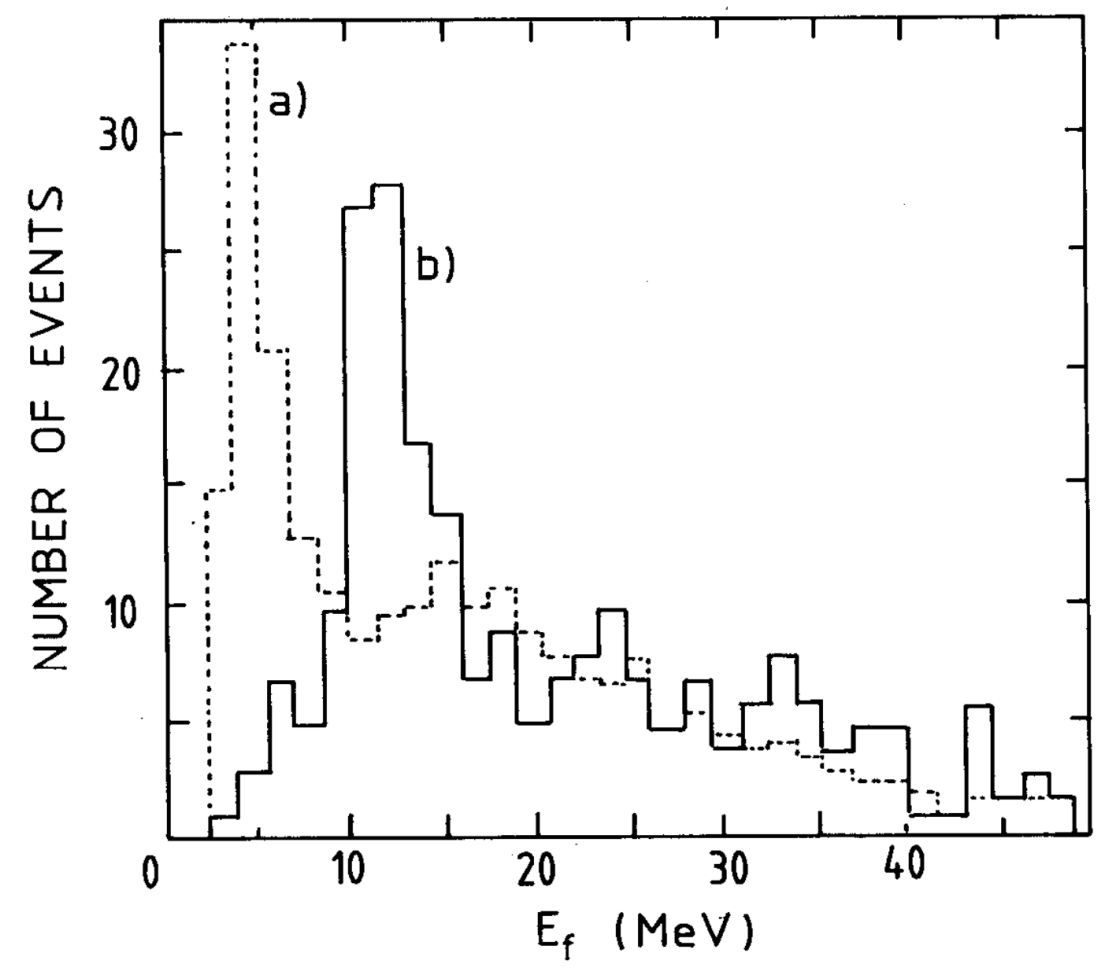

Figure 3.20. Energy deposition in CHARM detector.(a) electrons, (b) neutral pions, [50].

We apply this idea in the NOvA detectors, which means that the signature to distinguish electron from the neutral current $\pi^{0}$ is the energy depositions in the first few planes from the first hit. We then look into the $d E / d x$ in the first four planes.

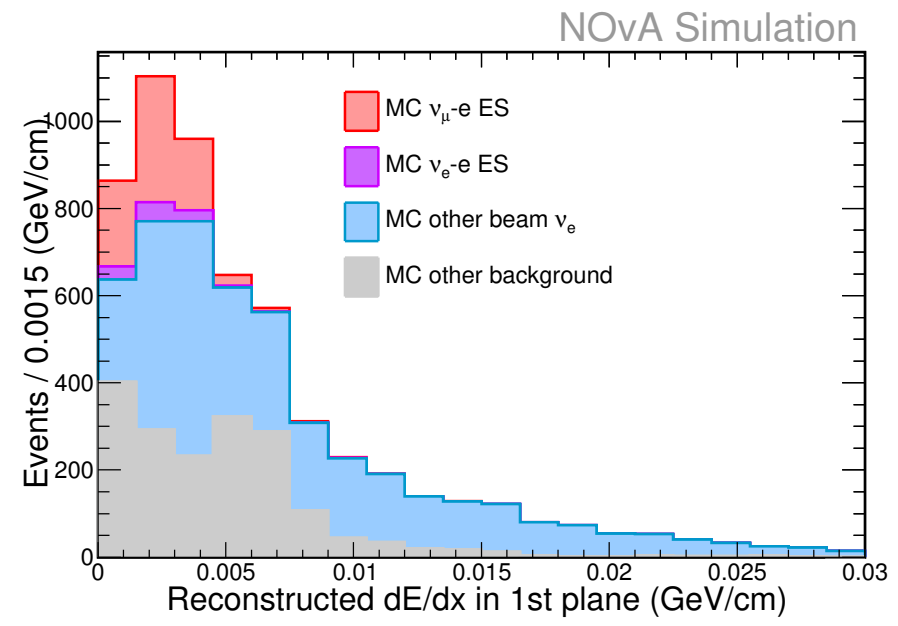

Figure 3.21. The $\mathrm{dE} / \mathrm{dx}$ distribution in the first plane. 


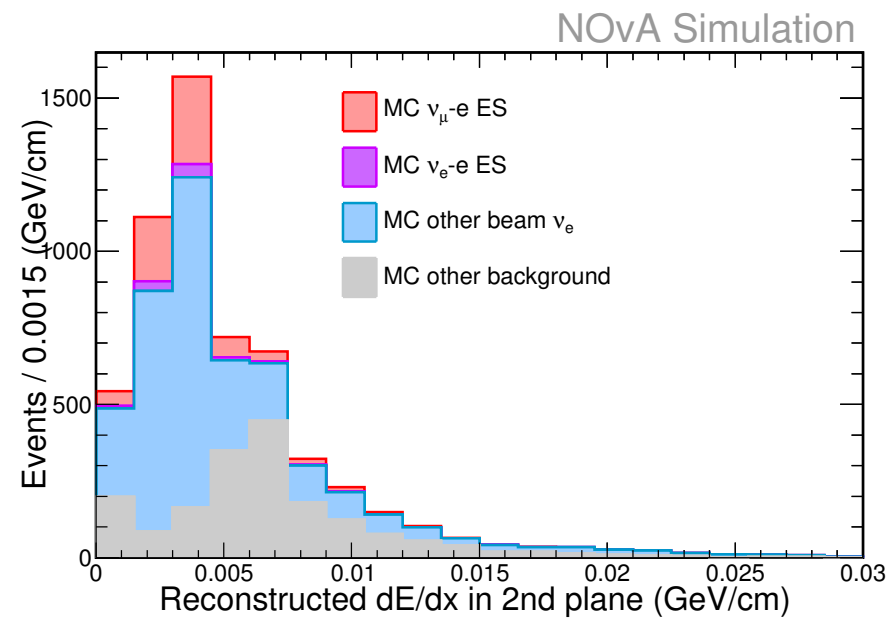

Figure 3.22. The $\mathrm{dE} / \mathrm{dx}$ distribution in the second plane.

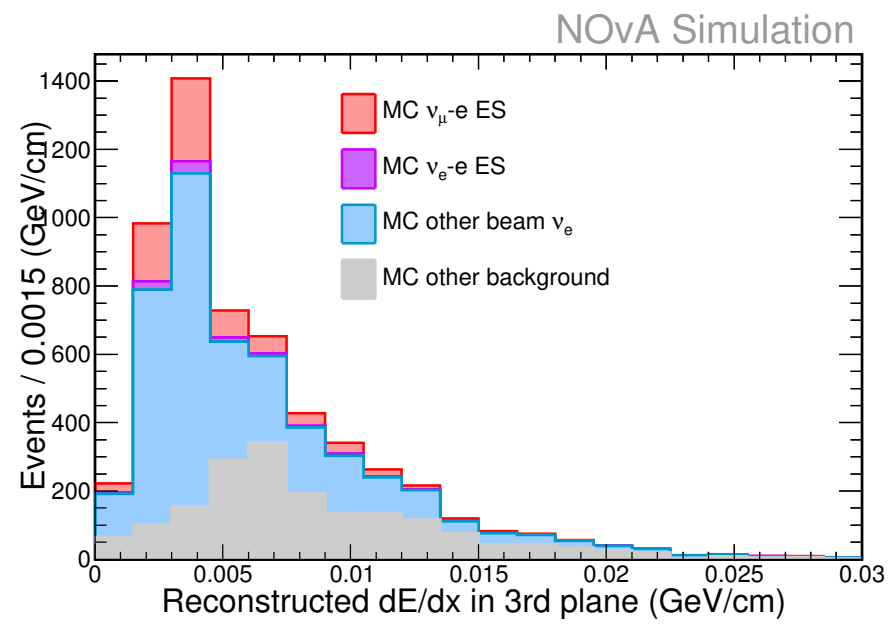

Figure 3.23. The $\mathrm{dE} / \mathrm{dx}$ distribution in the third plane. 


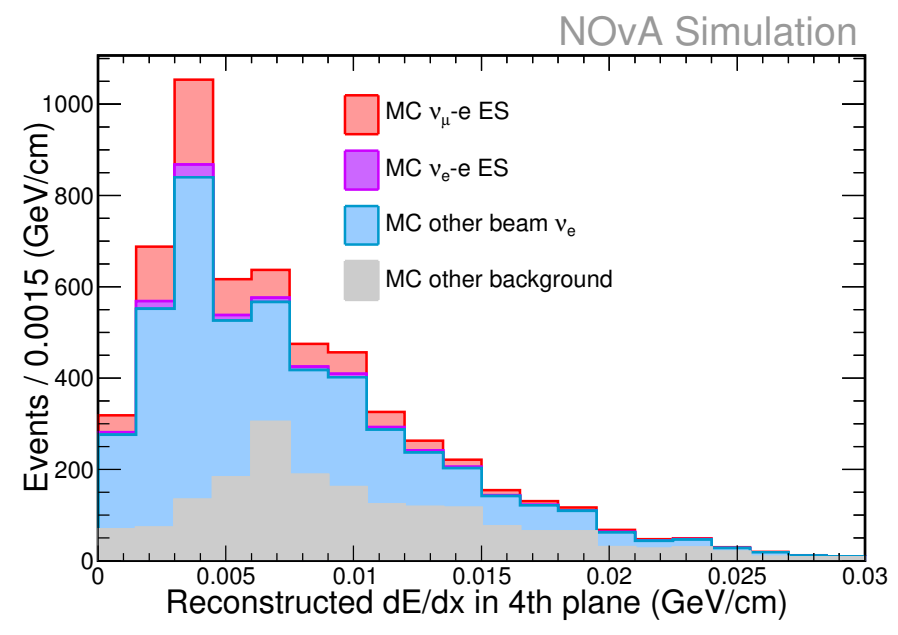

Figure 3.24. The $\mathrm{dE} / \mathrm{dx}$ distribution in the fourth plane.

From the figures shown above (Fig. 3.21, Fig. 3.22, Fig. 3.23 and Fig. 3.24), we realize that the $d E / d x$ distributions of $\nu$-e elastic scattering (ES) are similar as the other background. It is impossible to use any traditional selections on any of the $d E / d x$ in a certain plane. We have to build a deep learning neural network for the particle identification.

we use $\nu$-e elastic scattering events (GENIE:NuEElastic) as signal and neutral current coherent scattering events (GENIE:NCCOH) as background, then compare the $d E / d x$ in the first four planes to distinguish ES events from neutral pions [102]. Utilizing deep learning, we have to use multiple layers larger than three with much higher dimensions for the training nodes. Then the neural network can help us determine the veiled correlations between the $d E / d x$ in the first four planes and reject the background in a higher dimensional space.

The architecture of the three hidden layers is 25:12:6. These nodes ratios in each hidden layers are determined from enumerated practical tests and also from balancing the realistic computing time. As a result, for each inclusive Monte Carlo data file which contains 2000 simulation events, it could take more than six hours in the PID reconstruction process. And ten Monte Carlo inclusive files that nomally take only about three hours for NOvA's oscillation analysis might not be finished on grid within 24 computing hours. The performance of this PID is shown in Fig. 3.25. 


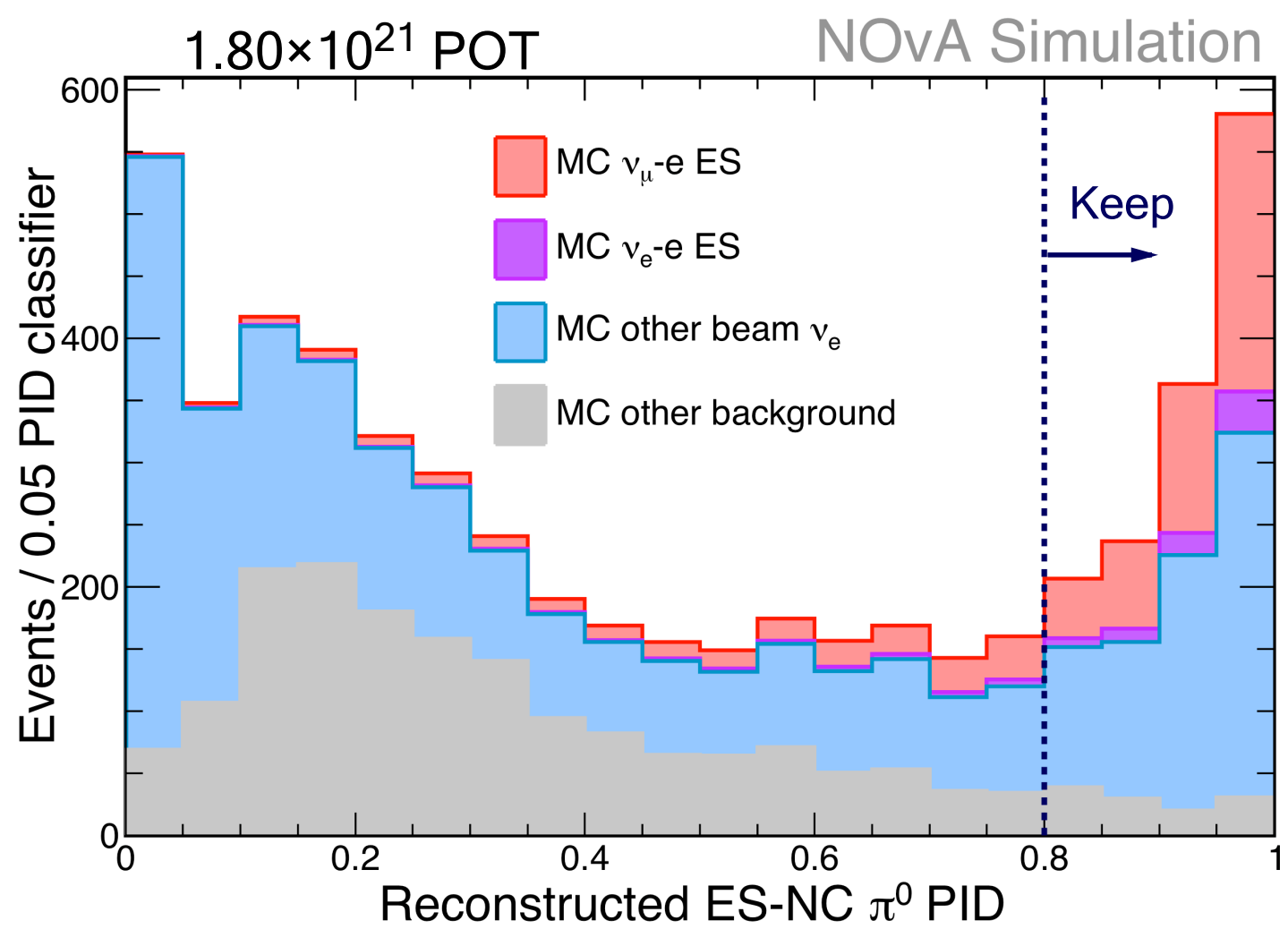

Figure 3.25. Performance for ES-NC $\pi^{0}$ PID. Plot is made after pre-selections and ES-CC PID $>0.9$.

By now we have the two PIDs for events selection (ES-CC PID > 0.9 and ES-NC $\pi^{0}$ PID $>0.8)$ to reject most of the background except the $\nu_{e}-N$ charge current events. We use the kinematic feature of $T \theta^{2}<2 m_{e}$ as the principle to remove $\nu_{e}-N$ events. 


\subsection{Rough Sensitivity for Neutrino Electron Elastic Scattering}

The After these cuts are made, we use two steps to tune the cuts for signal region of $\nu$-e magnetic scattering. The first step is to select the pure $\nu$-e weak scattering with the kinematic feature of $T \theta^{2}$, since the shower properties between elastic scattered electron and $\nu_{e} \mathrm{CC}$ shower can not be completely distinguished in $d E / d x$ comparison. We keep $\mathrm{T}>1.0$ $\mathrm{GeV}$ for now to keep the energy region dominated by $\nu_{\mu}$-e weak scattering through $\mathrm{Z}$ boson exchange. FOMs are considered as following.

- $s_{0}$ Sum of $\nu_{\mu}-e$ elastic scattering and $\nu_{e}-e$ elastic scattering events.

- $b_{0}$ Sum of all the non- $\nu$-e elastic scattering events in standard model MC.

- Optimize $T \theta^{2}$ cut for both $\operatorname{FOM}(1)=s_{0} / \sqrt{s_{0}+b_{0}}$ and $\operatorname{FOM}(2)=s_{0} / \sqrt{b_{0}}$.

\begin{tabular}{c|ccc|cc}
\hline Bins range & $\mathrm{MC} \nu_{\mu}-e$ & $\mathrm{MC} \nu_{e}-e$ & $\mathrm{MC}$ total & $s_{0} / \sqrt{s_{0}+b_{0}}$ & $s_{0} / \sqrt{b_{0}}$ \\
\hline 0 to 1 & 25.4 & 3.7 & 32.0 & 5.1 & 17.2 \\
0 to 2 & 43.8 & 6.4 & 57.3 & 6.6 & 18.8 \\
0 to 3 & 57.0 & 8.4 & 75.4 & 7.5 & 20.6 \\
0 to 4 & 66.4 & 9.8 & 89.4 & 8.1 & 21.0 \\
0 to 5 & 73.3 & 10.9 & 99.6 & 8.4 & 21.4 \\
0 to 6 & 78.2 & 11.7 & 108.4 & 8.6 & 20.9 \\
0 to 7 & 81.8 & 12.2 & 116.0 & 8.7 & 20.1 \\
0 to 8 & 84.6 & 12.6 & 121.1 & 8.8 & 19.9 \\
0 to 9 & 86.7 & 13.0 & 125.8 & 8.9 & 19.5 \\
0 to 10 & 88.3 & 13.2 & 129.4 & 8.9 & 19.2 \\
\hline
\end{tabular}

Table 3.3. Comparing significance for different $\mathrm{T} \theta^{2}$ cuts. Corresponding to Fig. 3.26

Traditionally, for a known cross section measurement, we should use $s_{0} / \sqrt{s_{0}+b_{0}}$ as the FOM, however, since the uncertainty for $\nu_{e} \mathrm{CC}$ interactions is at least 20 times larger than the $\nu$-e scattering, we must also use $s_{0} / \sqrt{b_{0}}$ to reject as much $\nu_{e} \mathrm{CC}$ as possible. As the same time, we keep the $s_{0} / \sqrt{s_{0}+b_{0}}$ value not far below the highest value. Finally, bin range from one to six is chosen in this case. 


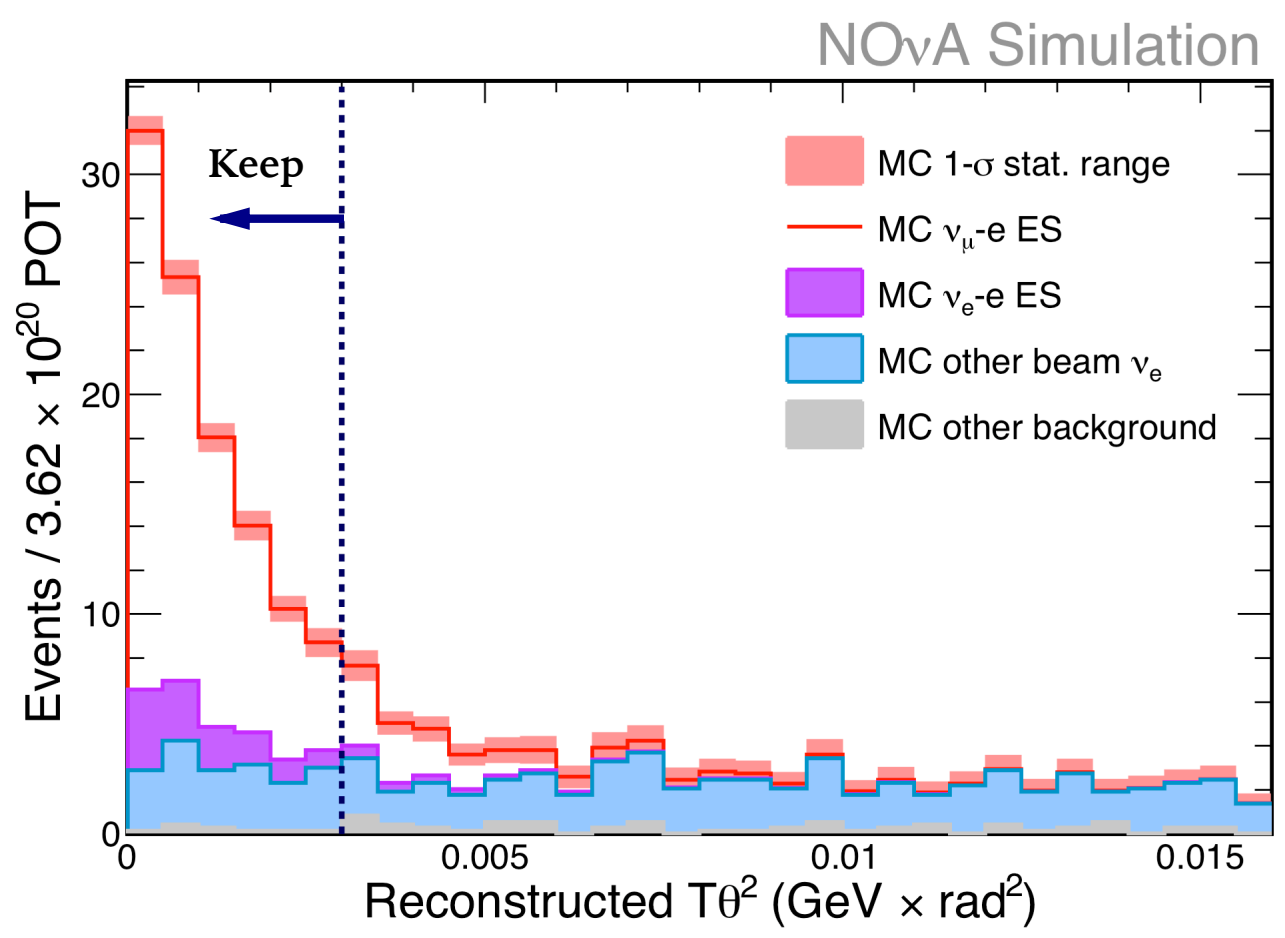

Figure 3.26. Tuning $T \theta^{2}$ cut for $T>1 \mathrm{GeV}$. See more discussions in Chapter four.

Which means $T \theta^{2}<0.003 \mathrm{GeV} \times \operatorname{Rad}^{2}$ is the signal region to select $\nu$-e scattering events from other background for $T>1.0 \mathrm{GeV}$. The second step is that we keep the $T \theta^{2}$ cut and release the $\mathrm{T}$ cut to estimate signal range of muon neutrino magnetic scattering. Then we look at the spectrum of recoil electron kinetic energy $\mathrm{T}$, and determine the rough signal region for the first analysis of muon neutrino magnetic moments. According to the discussion in Ref. [41], FOM is considered below.

- $s_{1}$ The MC magnetic scattering events due to NMM.

- $b_{1}$ The MC total events from SM interactions.

- $\sigma_{1}$ Adding up $1 \sigma$ statistical range and $10 \%$ systematic uncertainty.

- Optimize $\mathrm{T}$ region for $\mathrm{FOM}=s_{1} / \sqrt{b_{1}+\sigma_{1}^{2}}$.

So, to estimate the analysis region for muon neutrino magnetic moment, we have to consider the total uncertainty in a more serious way, since the magnetic scattering is a very 
rare interaction.

\begin{tabular}{c|cccc|c}
\hline Bins range & MC NMM & MC $\nu_{\mu}-e$ & MC $\nu_{e}-e$ & $\mathrm{MC} \mathrm{SM}$ & $s_{1} / \sqrt{b_{1}+\sigma_{1}^{2}}$ \\
\hline 7 to 15 & 14.06 & 70.17 & 5.24 & 86.31 & 0.930 \\
7 to 16 & 15.37 & 80.05 & 5.96 & 96.92 & 0.943 \\
7 to 17 & 16.61 & 90.14 & 6.71 & 107.76 & 0.950 \\
7 to 18 & 17.74 & 99.94 & 7.47 & 118.31 & 0.952 \\
7 to 19 & 18.84 & 110.11 & 8.27 & 129.98 & 0.943 \\
7 to 20 & 19.85 & 120.10 & 8.96 & 140.65 & 0.940 \\
\hline
\end{tabular}

Table 3.4. Tuning cut T for higher edge of NMM signal region. Corresponding to Fig. 3.27.

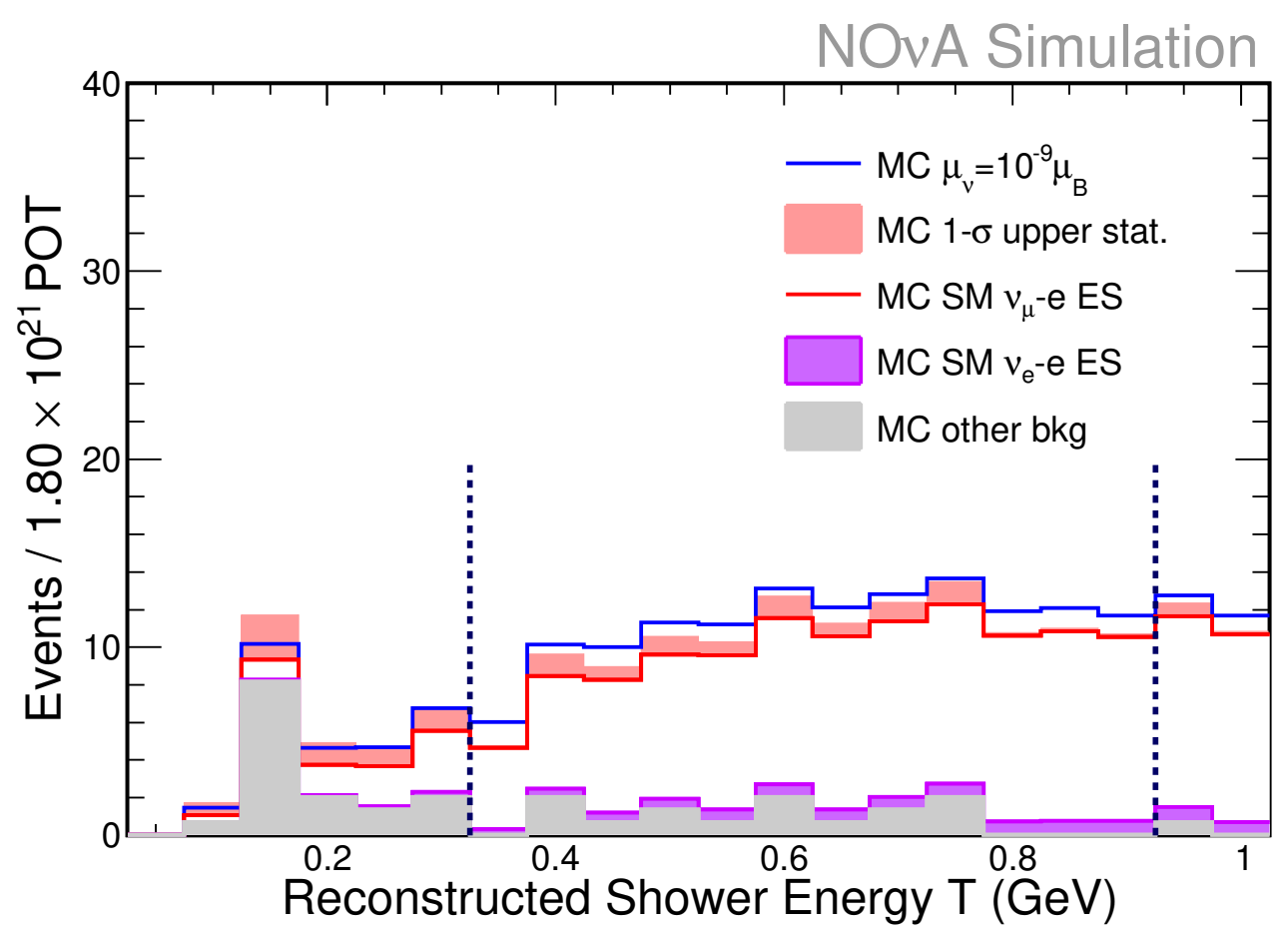

Figure 3.27. Tuning cut $\mathrm{T}$ for higher edge of the first analysis NMM signal region.

By now we determined the signal region for the first analysis of neutrino magnetic moment in NOvA near detector. In Chap. 5, we tune different components in MC to see if the statistic rates in this region is sufficient to counter the total systematic shifts.

In Fig. 3.27, we have to abandon the first 6 bins, not only because the NMM events are 
lower than $1 \sigma$ fluctuation of known hadronic showers, but also to remove possible hadronic showers that are not modeled in GENIE yet like neutral current meson exchange current (MEC) events (Fig. 3.28), an unmodeled hadronic components in neutrino-nucleus interaction.
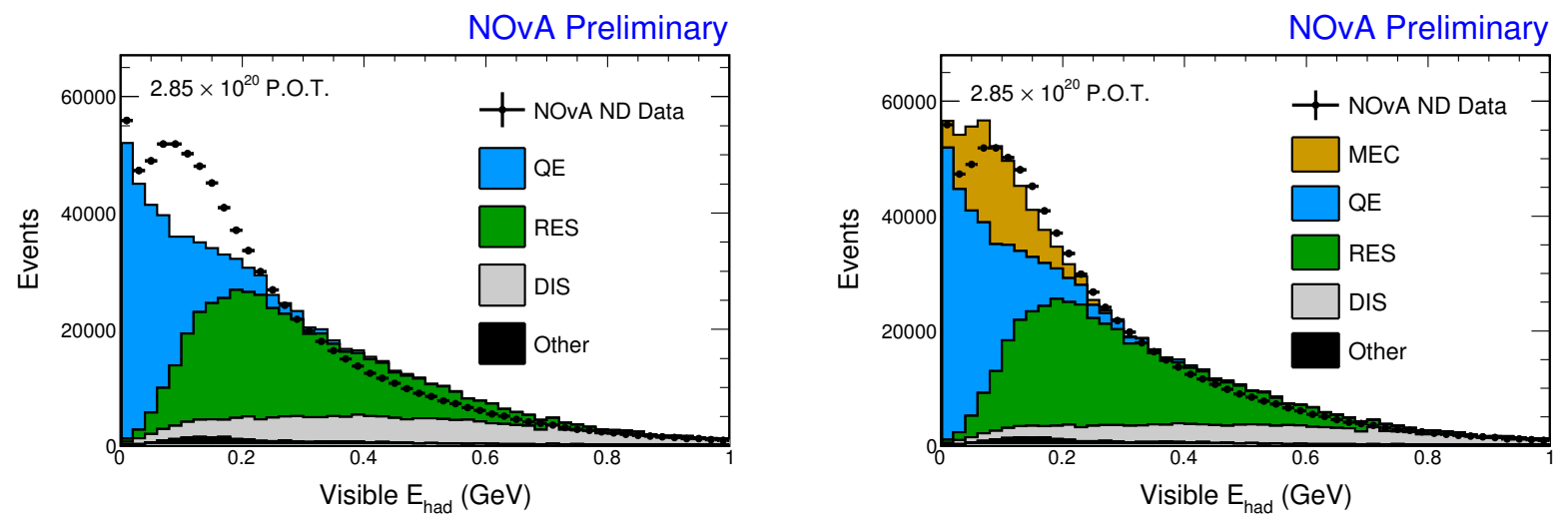

Figure 3.28. Visible hadronic energy distribution from selected ND muon neutrino events in NOvA first analysis. The right plot is added with non-resonance pion production reweight as charge current MEC events.

So that in next chapter, I will compare data/MC for $T>1 \mathrm{GeV}$ region and estimate all the possible systematic uncertainties in beam simulation. Then in Chap. 5, I will compare the data/MC in NMM signal region to setup the upper limit of muon neutrino magnetic moments. 
CHAPTER 4

Neutrino-Electron Elastic Weak Scattering

\subsection{Strategy for High-Energy Flux Constraint}

As mentioned earlier, for both weak and magnetic scattering, the cross section is proportional to neutrino energy. Thus, if the high energy neutrino tail is not modeled correctly in the simulation, it could cause significant systematic uncertainty. The relation between neutrino flux and the cross section is the following.

$$
\sigma=\frac{N-b}{A \epsilon \Phi}
$$

Here, the $\sigma$ is the cross section for a certain interaction channel, $\epsilon$ is the total acceptence and efficiency. $\mathrm{N}$ is the observed events in data, so that $\mathrm{N}-\mathrm{b}$ represents the selected signal for that interaction channel in data. $A$ represents the target numbers and $\Phi$ is the neutrino beam flux. So that if we have a well known cross section, we can use it as a data driven tool to constrain the neutrino flux.

Traditionally, we reconstruct the muon neutrino energy by adding up muon energy and hadronic energy as shown in Eq. 4.2.

$$
E_{\nu_{\mu}}=E_{\mu}+E_{h a d}
$$

And this works pretty well for muon neutrino energy less than $5 \mathrm{GeV}$ in NOvA's $\nu_{\mu}$ disappearance analysis [2] (Fig. 4.1) introduced in chapter one. 


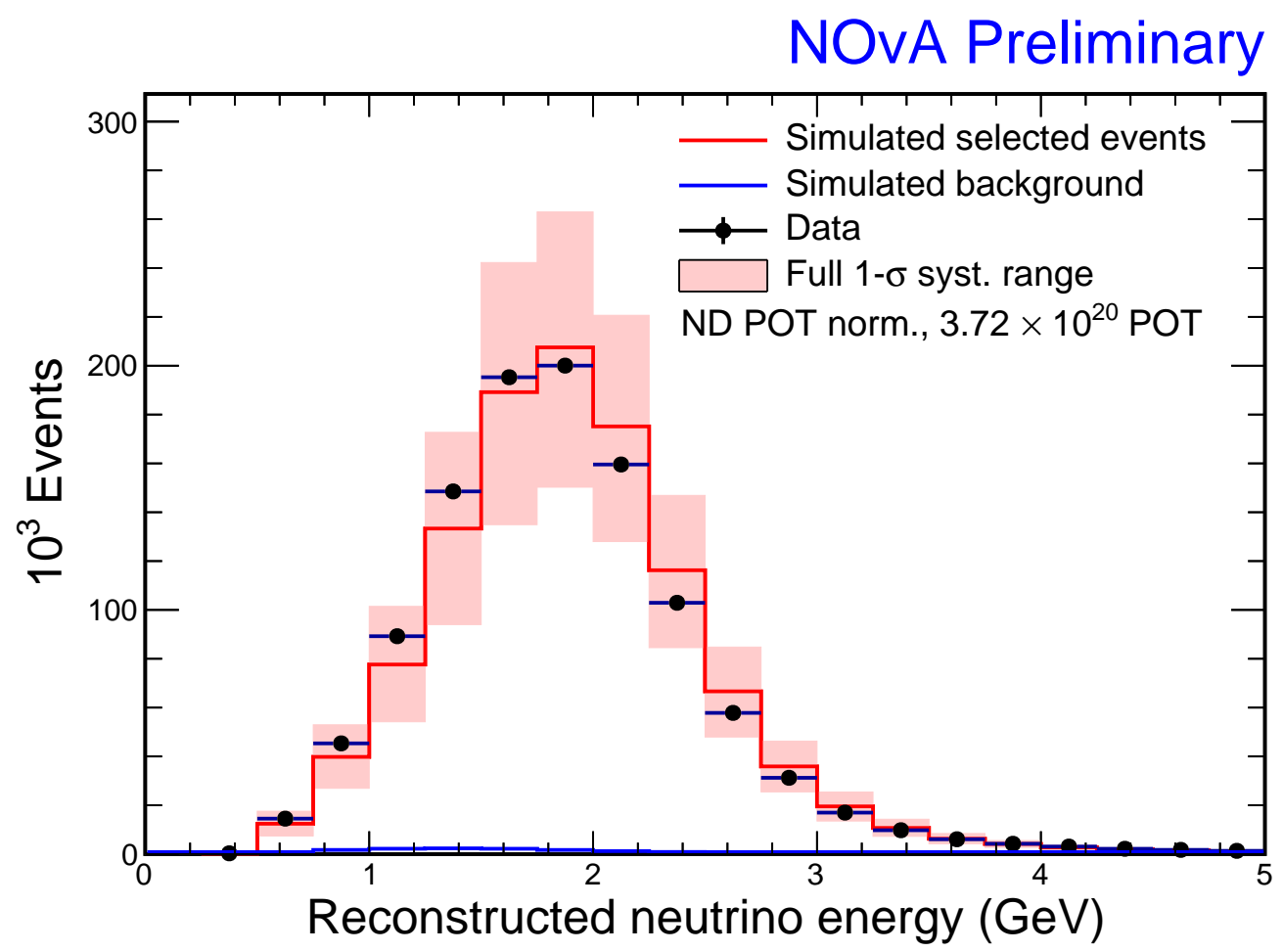

Figure 4.1. ND muon neutrino reconstructed energy distributions, data and MC with full systematics band, for neutrino energy, POT normalized.

However, for muon neutrino energy higher than $15 \mathrm{GeV}$, the dominant cross section is charge-current deep inelastic scattering (DIS). Thus, one may easily figure out that the traditional method may suffer from the following problems: There are too large uncertainties in the cross-section. The cross section for charge current DIS is not well known yet, nor is the angular distribution. The uncertainty of the cross section value is larger than the uncertainty of the neutrino beam flux, thus using this channel amplifies the systematic uncertainty of neutrino beam. The GENIE event generator only cover the uncertainty for $W<4 \mathrm{GeV}$ [82]. Here, the $W$ is the invariant hadronic mass of all secondary hadrons which is computed from the invariant 4-momentum transfer squared $Q^{2}[13]$.

$$
Q^{2}=2 E_{\nu}\left(E_{\mu}-p_{\mu}^{L}\right)-m_{\mu}^{2}
$$




$$
W^{2}=M^{2}+2 M\left(E_{\nu}-E_{\mu}\right)-Q^{2}
$$

And the $\mathrm{M}$ is the mass of nucleon, while $p_{\mu}^{L}$ is the longitudinal muon momentum. Even the data/MC energy spectrum match well, there is no guarantee that the neutrino beam is in the same direction as simulated.

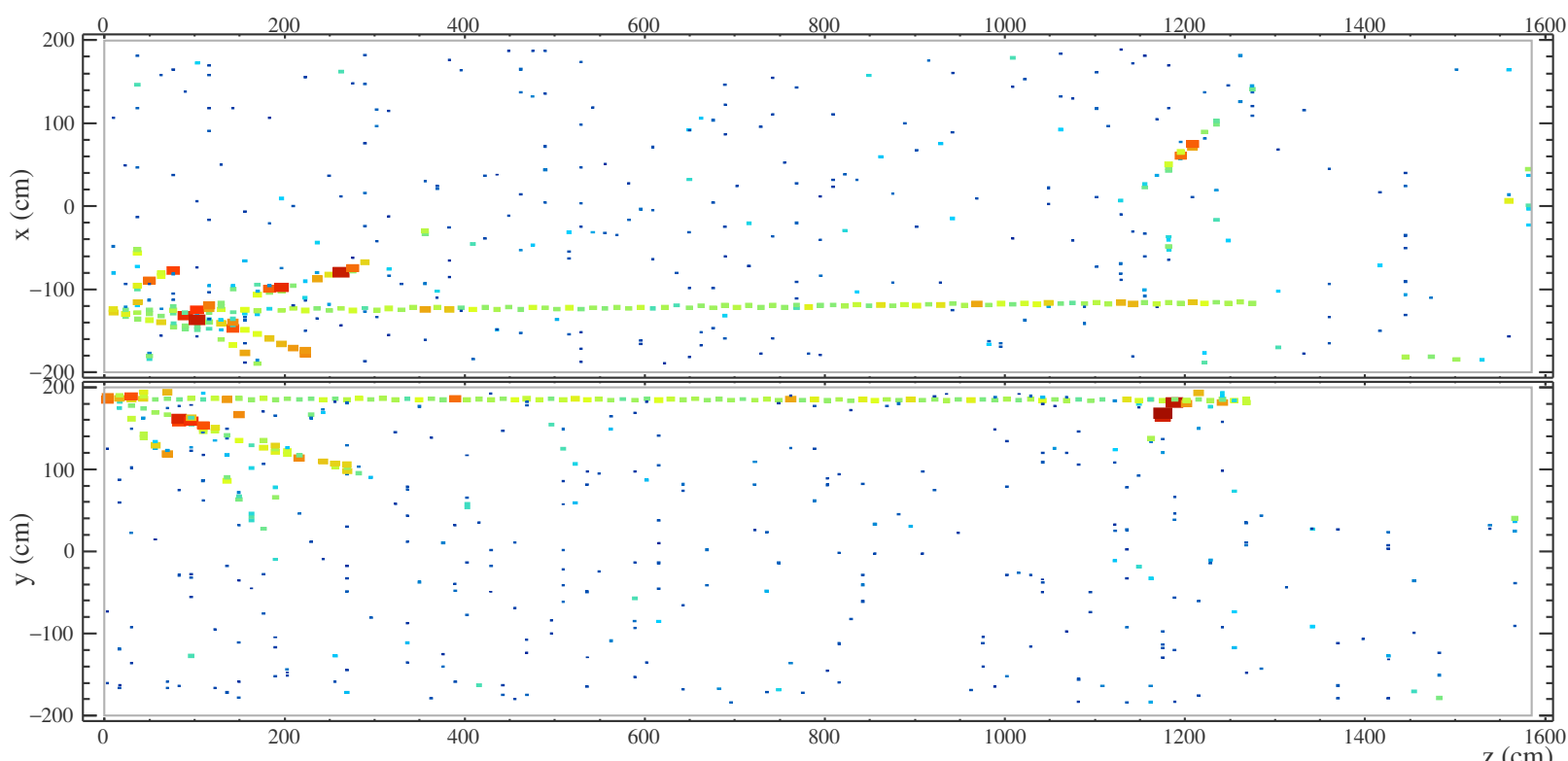

NOVA - FNAL E929 Run: $1201507111 / 1$ Event: 9 / --

UTC Thu Jan 1, 1970
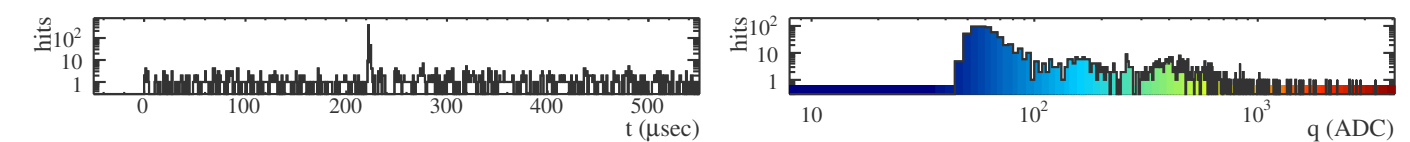

Figure 4.2. A typical charge current deep inelastic scattering in NOvA near detector. Note that the leading hadronic shower appearance is about 12 meters away from the interaction vertex.

It is hard to control the fiducial volume. In the deep inelastic scattering, one of the quarks inside the targeted nucleon is kicked out by the incident neutrino and forms hadronization after a flying time. The internal flying time for this leading hadronic shower may cause it been separated as an independent cluster, also the fragmentation of the rest of that nucleon is easily to be dispersed out of the detector. Another case is that the leading hadronic shower can be within the near detector volume but the vertex or the muon track is outside 
the detector in the rock. Either way, the loses of the reconstructed energy is not negligible.

Also, the nuclear effect is too large. For the non-resonance pion productions, meson exchange current (MEC) behave proportional to DIS process. The interaction between nucleons for atomic number higher than 12 is still a puzzle need to be solved. Thus, if we use a CC inclusive method for neutrino energy constraint, the reconstructed muon neutrino energy spectrum could shift to lower or higher due to incorrect hadronic energy estimation for MEC events.

In contrast, the $\nu_{\mu}$-e scatterings result in events that avoid most of such problems. The event keeps its property as a clean pure leptonic shower free from nuclear effects and easy to reconstruct and identify.

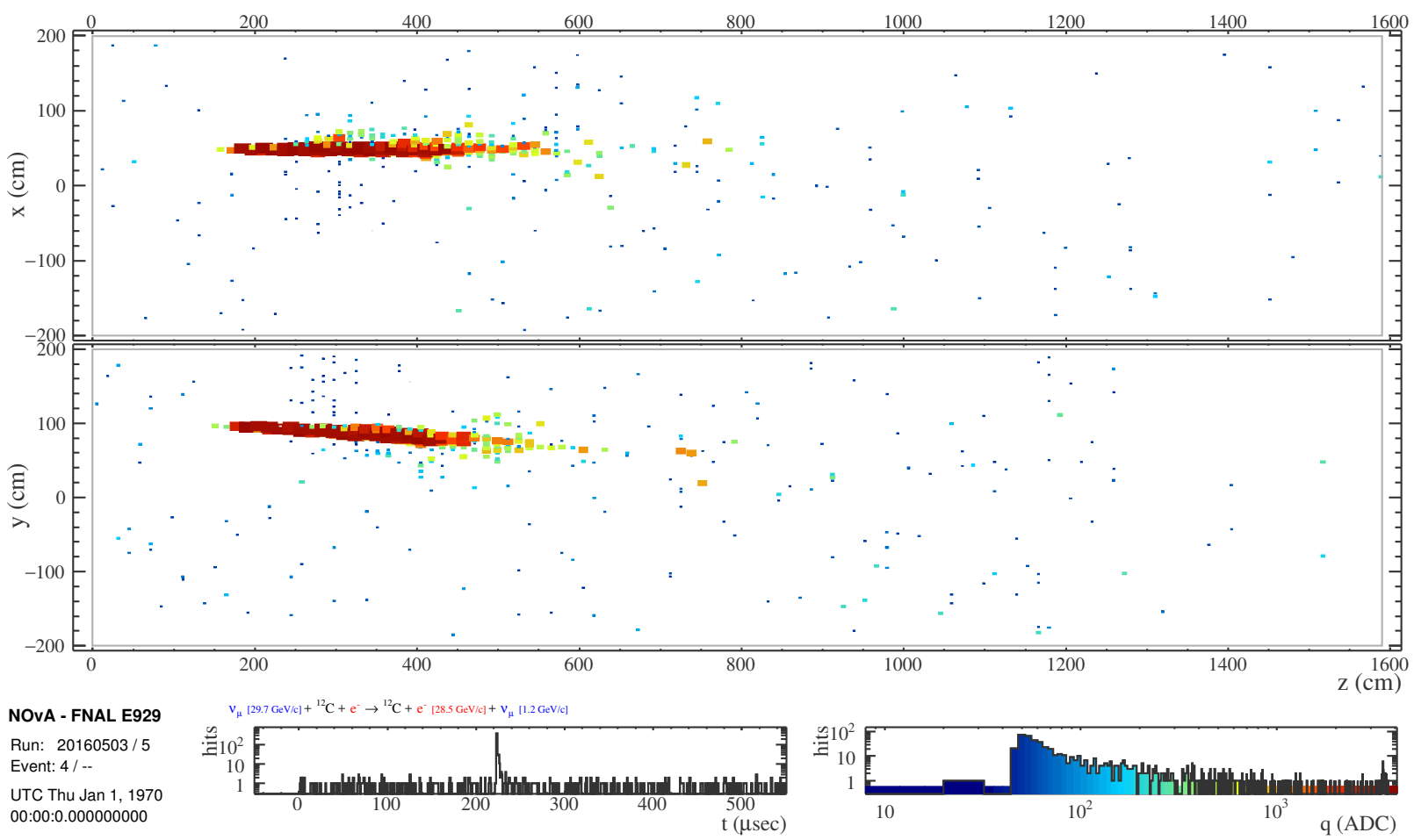

Figure 4.3. A scattered electron $(28.5 \mathrm{GeV})$ produced by a high energy muon neutrino (29.7 $\mathrm{GeV}$ ) interaction in NOvA near detector.

Thus, we believe that at present time, the most reliable way to constrain the high energy neutrino is to use the $\nu$-e elastic scattering, though we need to accumulate more events to 
get a solid statement since the cross section compared to overall neutrino nucleus scattering is tiny (Eq. 4.5).

$$
\frac{\sigma_{\nu-e}}{\sigma_{\nu-N}} \approx 10^{-4}
$$

In chapter five, we demonstrated that these high energy neutrinos may only take less than two percent in the overall uncertainty. However, the technique is crucial for lightweight dark matter searches since the $\nu_{e}$ charge-current interactions and $\nu_{\mu}$-e scattering from parent $K^{+}$decays dominate the major background of recoil electrons from lightweight dark matter scattering through a portal mediator $[47,102]$ as shown in Fig. 4.4.

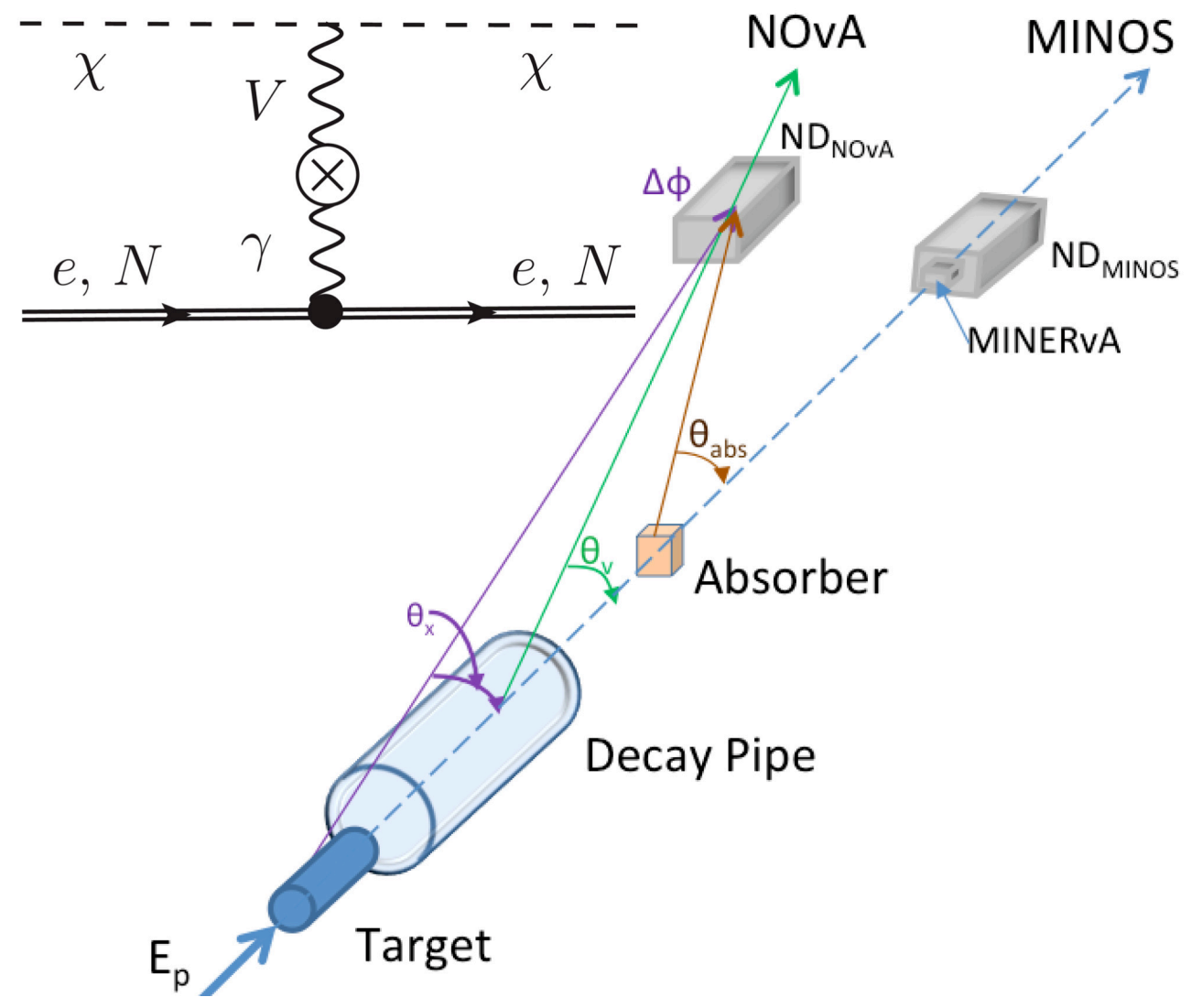

Figure 4.4. The diagram for lightweight dark matter (LDM) produced in the proton on target collision and scatter through a mediator in the NOvA ND. 


\subsection{Data Analysis for Flux Constraint}

We have derived the relationship for general $\nu$-e elastic scattering in section 2.1, that

$$
T \theta^{2}=2\left(1-\frac{T}{E_{\nu}}\right) m_{e}=2(1-y) m_{e}
$$

Therefore, no matter what kind of $T$ and $E_{\nu}$ combination, we always have $T \theta^{2}<2 m_{e}$. We use this kinematic feature to distinguish electrons from $\nu$-e elastic scattering from those electrons caused by $\nu_{e^{-}} N$ charge current process that have occasionally passed the PID selections.

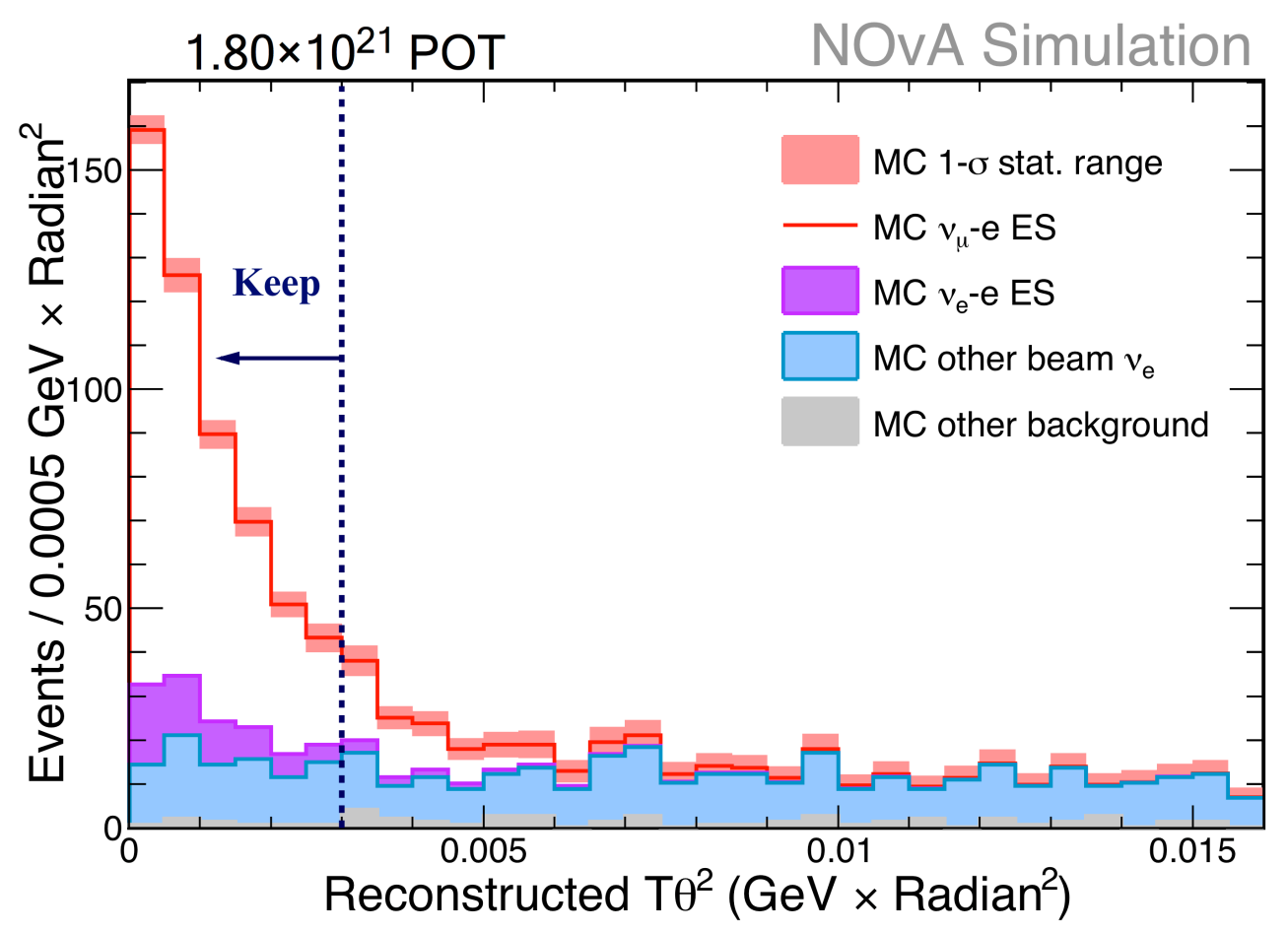

Figure 4.5. ND muon neutrino electron elastic scattering (ES) reconstructed $T \theta^{2}$ distributions, the optimal cut is chosen at $T \theta^{2}<0.003 \mathrm{GeV} \times \operatorname{Radian}^{2}$.

Firstly, we introduce the strategy for selecting methods, suppose the number of events that we want to keep is $s_{\text {keep }}$ and the other events we want to reject is $b_{\text {other }}$. Normally, people use $\frac{s_{k e e p}}{\sqrt{s_{\text {keep }}+b_{\text {other }}}}$ as the figure of merit (FOM) to determine the range of selection parameters for a well known process. However, in our case, we also have to include another FOM which is $\frac{s_{\text {keep }}}{\sqrt{b_{\text {other }}}}$. There are several reasons to do so. 
The most obvious reason is that the $\nu_{e}-N$ charge current events have much larger uncertainties in the cross section, and their influence of the uncertainty of the result must be minimized. A deeper reason is that after the two PID selections, we do not know what distribution should the $\nu_{e}-N$ charge current events looks like in the $T \theta^{2}$. Also, based on that, we never know what distribution the events rate in each bin of the $T \theta^{2}$ should following.

The datasets in the NOvA near detector of this analysis is the following:

- Data $3.62 \times 10^{20} \mathrm{POT}$,

- MC $\nu$-e sample $6.74 \times 10^{23} \mathrm{POT}$,

- MC inclusive dataset $2.64 \times 10^{21}$ POT that used in Ref. [94].

All the Monte Carlo simulation samples are scaled to data POT $\left(3.62 \times 10^{20} \mathrm{POT}\right)$ in this thesis.

The practical difficulties also force us do so. The $\nu_{e}-N$ charge current events sample is from the standard inclusive Monte Carlo files for NOvA second analysis, which is about 7 times of the data sample after reconstruction. While the $\nu$-e elastic scattering sample is scaled down from dataset with around more than 2000 times of data POT. Thus the $\nu_{e^{-}} N$ charge current events suffered much more from the statistical fluctuation. Also, when we build the FOM for a certain analysis, we should take the corresponding distribution into account. In Fig. 4.5, we found that $F O M=\frac{s_{\nu-e}}{\sqrt{s_{\nu-e}+b_{\text {other }}}}$ were not dramatically change so that indicate this FOM may not chosen suitable for this $T \theta^{2}$ distribution. Therefore, we have to balance this selection with another FOM, which is $\frac{s_{\nu-e}}{\sqrt{b_{o t h e r}}}$.

We have to keep the data blinded at this stage, so that it protects the selection from the biased judgments. After we made this $T \theta^{2}$ cut, we are safe to look into data and see comparison with the standard model prediction in MC. 


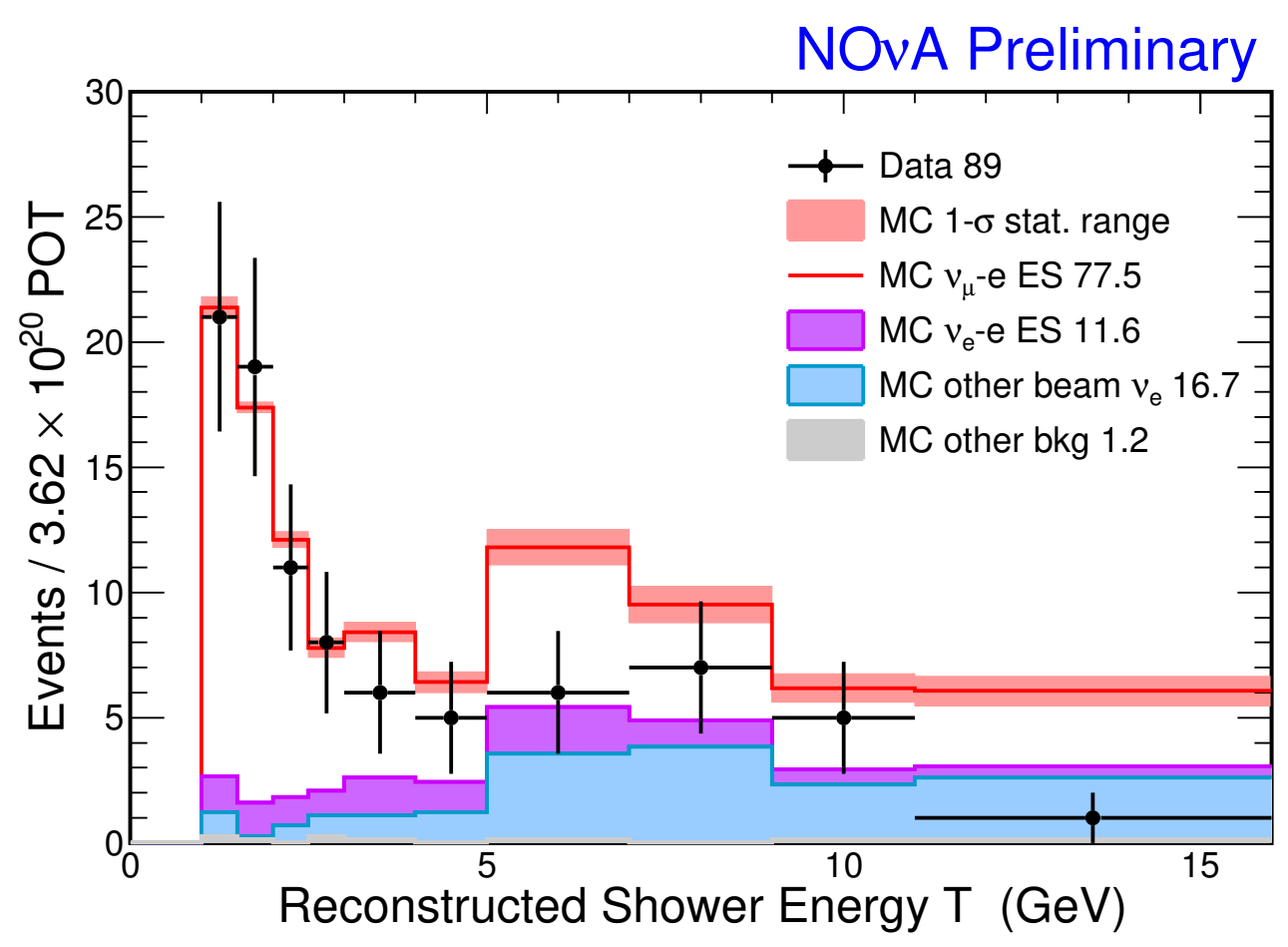

Figure 4.6. ND muon neutrino electron elastic scattering reconstructed $\mathrm{T}(\mathrm{GeV})$ distributions, after $T \theta^{2}<0.003 \mathrm{GeV} \times$ Radian $^{2}$ cut shown in Fig. 4.5.

The events rate $R(T)$ in each reconstructed energy $(T)$ bin directly reflects the neutrino beam flux since the cross section $\frac{d^{2} \sigma}{d T d E_{\nu}}$ is well-known and modeled in the GENIE generator, and it has the proportional relation as

$$
R(T) \propto \int_{T}^{E_{\max }} \Phi\left(E_{\nu}\right) \frac{d^{2} \sigma}{d T d E_{\nu}} d E_{\nu}
$$

For the oscillation analysis, the neutrino energy is peaked around $2 \mathrm{GeV}$, so that we estimate the number of events in the recoil electron energy range from one to three $\mathrm{GeV}$.

\begin{tabular}{l|cccc}
\hline T range $(\mathrm{GeV})$ & $1.0-1.5$ & $1.5-2.0$ & $2.0-2.5$ & $2.5-3.0$ \\
\hline Data & 21 & 19 & 11 & 8 \\
MC $\nu$-e & 20.1 & 17.1 & 11.4 & 6.7 \\
MC total & 21.4 & 17.4 & 12.1 & 7.8 \\
\hline
\end{tabular}

Table 4.1. Comparing the data to prediction for $1 \mathrm{GeV}<\mathrm{T}<3 \mathrm{GeV}$. 
For the cross section measurement, we examine the scattering electron's energy from one to five GeV since those cross sections are roughly proportional to the neutrino energy and thus the average neutrino energy is about three $\mathrm{GeV}$.

\begin{tabular}{l|cccccc}
\hline T range $(\mathrm{GeV})$ & $1.0-1.5$ & $1.5-2.0$ & $2.0-2.5$ & $2.5-3.0$ & $3.0-4.0$ & $4.0-5.0$ \\
\hline Data & 21 & 19 & 11 & 8 & 6 & 5 \\
MC $\nu$-e & 20.1 & 17.1 & 11.4 & 6.7 & 7.3 & 5.2 \\
MC total & 21.4 & 17.4 & 12.1 & 7.8 & 8.4 & 6.4 \\
\hline
\end{tabular}

Table 4.2. Data/MC comparison for cross section interested range.

Since there are only two bins with $0.5 \mathrm{GeV}$ interval that have about 20 events around 20, the statistic to date is not sufficient to make an advanced data driven constraint. Thus, we study the integral of events in those bins and find that the data and Monte Carlo are within $1 \sigma$, agree very well .

It is possible that the criteria for selecting events with $T<5 \mathrm{GeV}$ is too tight for higher energy events. To evaluate this lost efficiency for high energy events. We put the fiducial volume back to a larger space with $100 \mathrm{~cm}<\mathrm{Z}<1200 \mathrm{~cm}$. And keep the shower property cuts the same with the same kinematic selection in Fig. 4.5. Thus we get the results in Table 4.3 and in Fig. 4.7.

\begin{tabular}{l|llllllllll}
\hline $\mathrm{T}(\mathrm{GeV})$ & $1.0-1.5$ & $1.5-2.0$ & $2.0-2.5$ & $2.5-3.0$ & $3.0-4.0$ & $4.0-5.0$ & $5.0-7.0$ & $7.0-9.0$ & $9.0-11.0$ & $11.0-16.0$ \\
\hline Data & 24 & 21 & 12 & 8 & 7 & 6 & 6 & 8 & 5 & 9 \\
$\mathrm{MC} \nu-e$ & 23.0 & 19.6 & 13.2 & 7.8 & 8.6 & 6.2 & 9.8 & 6.9 & 4.8 & 4.3 \\
MC total & 24.5 & 20.2 & 14.0 & 8.8 & 9.6 & 7.6 & 14.0 & 11.3 & 7.6 & 7.8 \\
\hline Data/MC & 0.98 & 1.04 & 0.86 & 0.90 & 0.72 & 0.79 & 0.43 & 0.71 & 0.65 & 1.16 \\
\hline
\end{tabular}

Table 4.3. The data/MC for $T>1 \mathrm{GeV}$ and $100 \mathrm{~cm}<\mathrm{Z}<1200 \mathrm{~cm}$. 


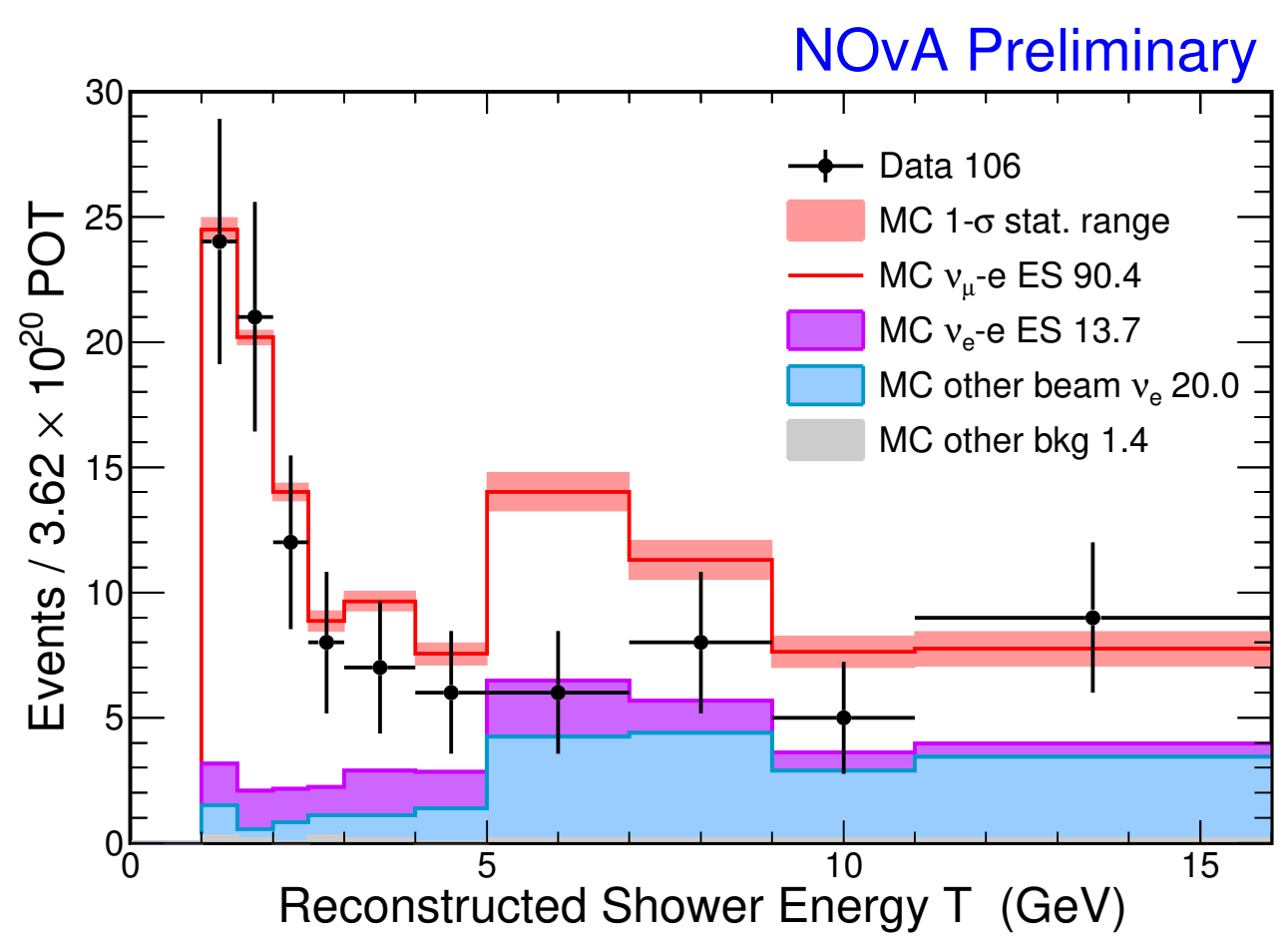

Figure 4.7. ND muon neutrino electron elastic scattering reconstructed $\mathrm{T}(\mathrm{GeV})$ distributions, after $T \theta^{2}<0.003 \mathrm{GeV} \times$ Radian $^{2}$ cut with $100 \mathrm{~cm}<\mathrm{Z}<1200 \mathrm{~cm}$ fiducial volume.

In this plot, we see good agreements everywhere except the bin of 5 to $7 \mathrm{GeV}$. It is within tolerance that one of the ten bins varies about $2 \sigma$ in general.

To analyze the details about this plot. We discuss the $\nu_{\mu}$ components and $\nu_{e}$ components respectively.

For the $\nu_{\mu}$ induced events, most of them are from $\nu_{\mu}$ e elastic scattering. We illustrate the relationship between true $\nu_{\mu}$ neutrino energy and the neutrino ancestor in Fig. 4.8. 


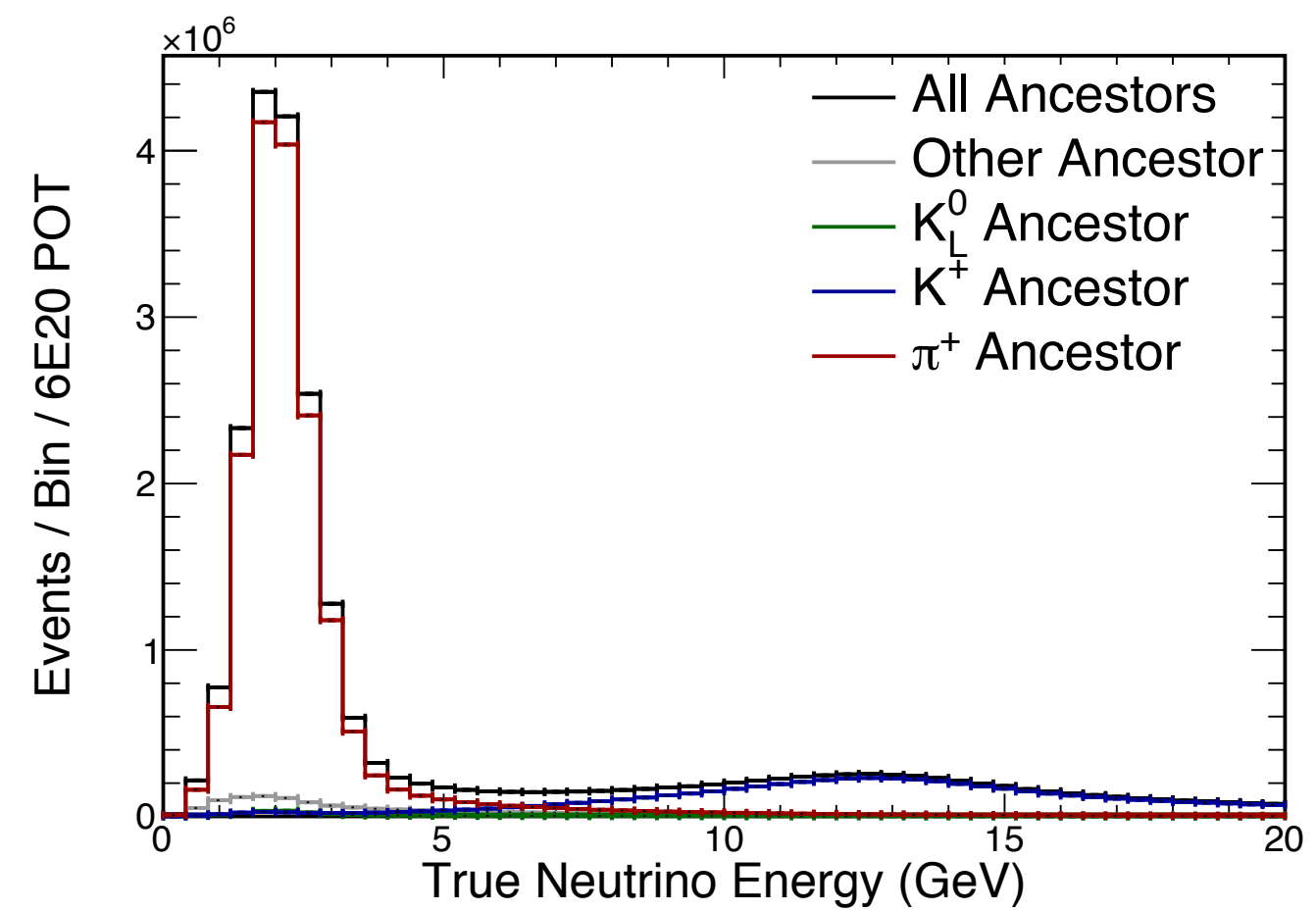

Figure 4.8. ND muon neutrino spectrum with ancestors. The $K^{+}$dominated for neutrino energy larger than six GeV.

Most of the $\nu_{\mu}$ for $E_{\nu}>5 \mathrm{GeV}$ are produced from $K^{+}$decays. And beam $\nu_{e}$ also from $K^{+}$decays. But there are two many nuisance parameters in this issue. The angular $K^{+}$ production parameter [73], the non-standard interaction effects $[40,86]$ and the unknown $\nu_{e}$ interference might all count.

We do see that the data/MC agree well for $T>11 \mathrm{GeV}$, and the previous deficit shown in Fig. 4.6 might just arise from improper vertex region for high energy electrons. That support the bump between 10 to $15 \mathrm{GeV}$ in Fig. 4.8 is modeled accurately in beam simulation.

In general, the data and Monte Carlo agree within $2 \sigma$ for $K^{+}$and $K_{L}^{0}$ decayed neutrinos by looking at data and Monte Carlo comparison for $T>5 \mathrm{GeV}$ region.

The results for $T>1 \mathrm{GeV}$ region have shown that the selection efficiency and flux simulation agree well in data and standard model Monte Carlo. However, due to limited statistic, we are not able to constrain the uncertainty of the neutrino flux. So that in next 
section, we estimate the flux shifts by tuning each parameter in the beam simulation for NOvA second analysis.

\subsection{Systematic Uncertainties of Beam Simulation}

The neutrino beam systematic uncertainty is mainly coming from the uncertainty of the flux of pions and kaons produced in the NuMI target. As shown in the $T$ distribution of Fig. 4.9 for the $\nu$-e elastic weak scattering, the pion production of NuMI beam is modeled well. We treat the flux uncertainty from $K^{+}$decay by tuning the $\mathrm{MC}$ by $\pm 20 \%$, which is discussed in chapter five.

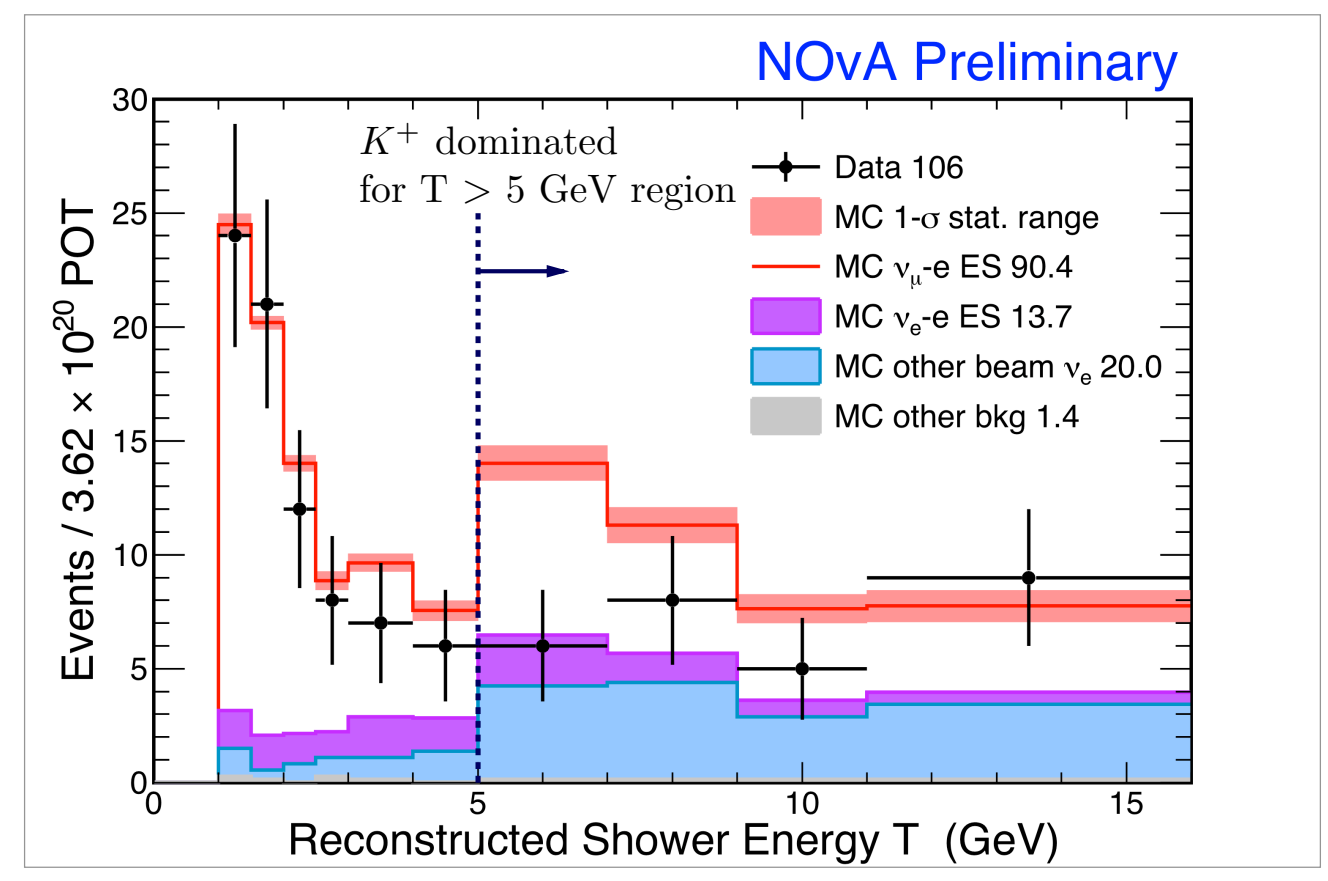

Figure 4.9. Reconstructed electron energy distribution for muon neutrino on electron elastic scattering for electron recoil energy $\mathrm{T}>1 \mathrm{GeV}$. 


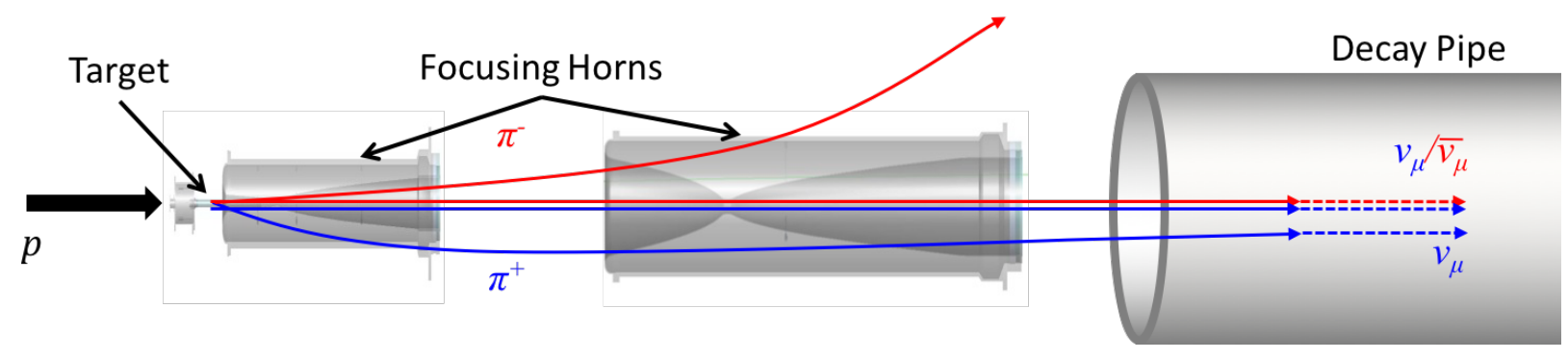

Figure 4.10. The diagrams of the target, horns, and start of the decay pipe (not to scale) that shows $\pi^{+}$and $K^{+}$being focused to create neutrinos in forward horn current (FHC) mode.

For the $\pi^{+}$production part, we estimate the flux uncertainties from NOvA beam simulation. Fig. 4.10 and Fig. 4.11 show the complicated system of the neutrino beam production. The nominal conditions for the beam simulation are the following items:

- Horn Current $200 \mathrm{kA}$

- Default Positions of Horns and Target

- linear Magnetic Field Distribution

- Beam Spot Size $1.1 \mathrm{~mm}$ in X\&Y

- Cooling Water layer 


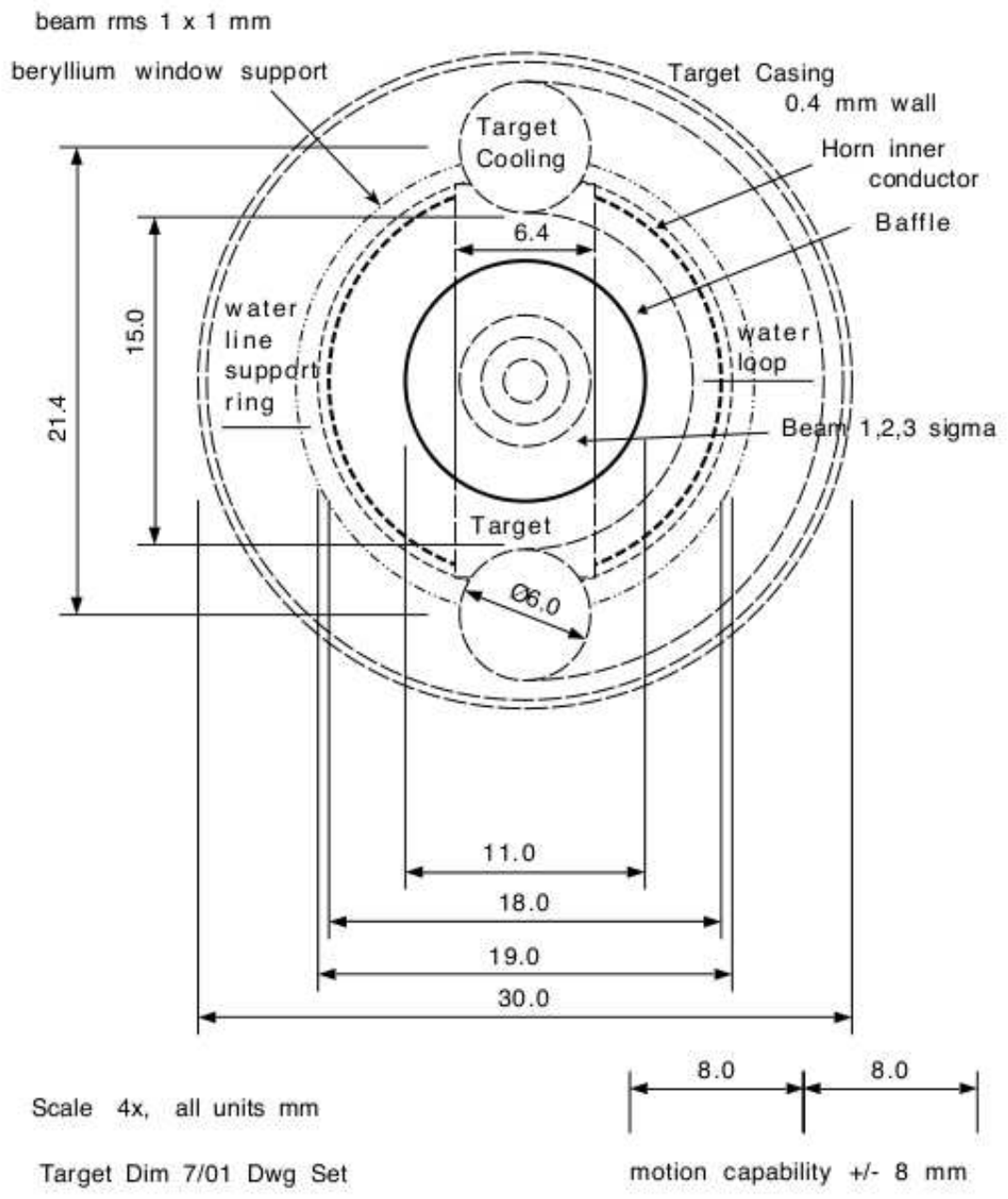

Figure 4.11. This figure shows what the proton beam sees as it travels through the NuMI baffle and hits the target. This plot is originally from Ref. [4]

We shift each above parameter of the parent $\pi^{+}$beam simulation within the maximum physical range to get each corresponding uncertainty. We then sum them in quadrature as shown in Table. 4.4 to get the systematic uncertainty of $\pi^{+}$flux. 


\begin{tabular}{l|c|c}
\hline Systematic shifts for parent $\pi^{+}$ & $0 \leq E_{\nu} \leq 10 \mathrm{GeV}$ & $0 \leq E_{\nu} \leq 3 \mathrm{GeV}$ \\
\hline Standard (fluka 2011.2b.6) & $0 \%$ & $0 \%$ \\
Horn current $\pm 1 \mathrm{kA}$ & $0.08 \%$ & $0.22 \%$ \\
Horn 1 X\&Y $\pm 2 \mathrm{~mm}$ & $2.04 \%$ & $1.71 \%$ \\
Horn 2 X\&Y $\pm 2 \mathrm{~mm}$ & $0.51 \%$ & $0.52 \%$ \\
Beam position on the target $\pm 0.5 \mathrm{~mm}$ in X & $0.68 \%$ & $0.68 \%$ \\
Beam position on the target $\pm 0.5 \mathrm{~mm}$ in Y & $0.43 \%$ & $0.36 \%$ \\
Target position Z \pm 7 mm & $1.19 \%$ & $1.18 \%$ \\
Beam spot size 0.9 mm to 1.5 mm & $2.64 \%$ & $3.11 \%$ \\
Exponential B field in the horn skin & $0.00 \%$ & $0.02 \%$ \\
Water layers 1 mm as maximum & $4.60 \%$ & $5.76 \%$ \\
\hline Quadrature & $5.89 \%$ & $6.93 \%$ \\
\hline
\end{tabular}

Table 4.4. Shifting each parameter by its maximum physical range in the beam simulation to estimate the flux uncertainties from parent $\pi^{+}$. For further details, see Ref. [85].

The horn magnetic field in the default model decreases linearly in the horn skin while the shifted one assumes the magnetic field decrease exponentially in the $0.77 \mathrm{~cm}$ horn skin. The largest uncertainty comes from the water layer assumption. The horn conductor is consistently sprayed by cooling water which produce a surrounding thin water layer on the horn. This causes an average five percents uncertainty in the total flux due to the meson absorption in the water layer [9].

We use the quadrature result from the column of $0 \leq E_{\nu} \leq 10 \mathrm{GeV}$ from Table 4.4 as the systematic uncertainty of parent $\pi^{+}$systematic error and apply it in the analysis of neutrino magnetic moment. 
CHAPTER 5

Analysis of Muon Neutrino Magnetic Moment

\subsection{Systematic Uncertainties and Selection Optimization}

In this section, we examine the validation of the selection region for the most important systematic uncertainties. The uncertainties mainly originate from two types of sources, the selection efficiencies and the intrinsic modelings in the Monte Carlo (MC) simulation. We regard the number of events for both signal and SM background in Fig. 5.1 as a baseline. We then calculate each systematic shift in the events yield in turn for both $\nu_{\mu}$-e electromagnetic (EM) elastic scattering (ES) and the SM prediction in Monte Carlo. We sum the uncertainties in quadrature to obtain the total systematic uncertainty. 


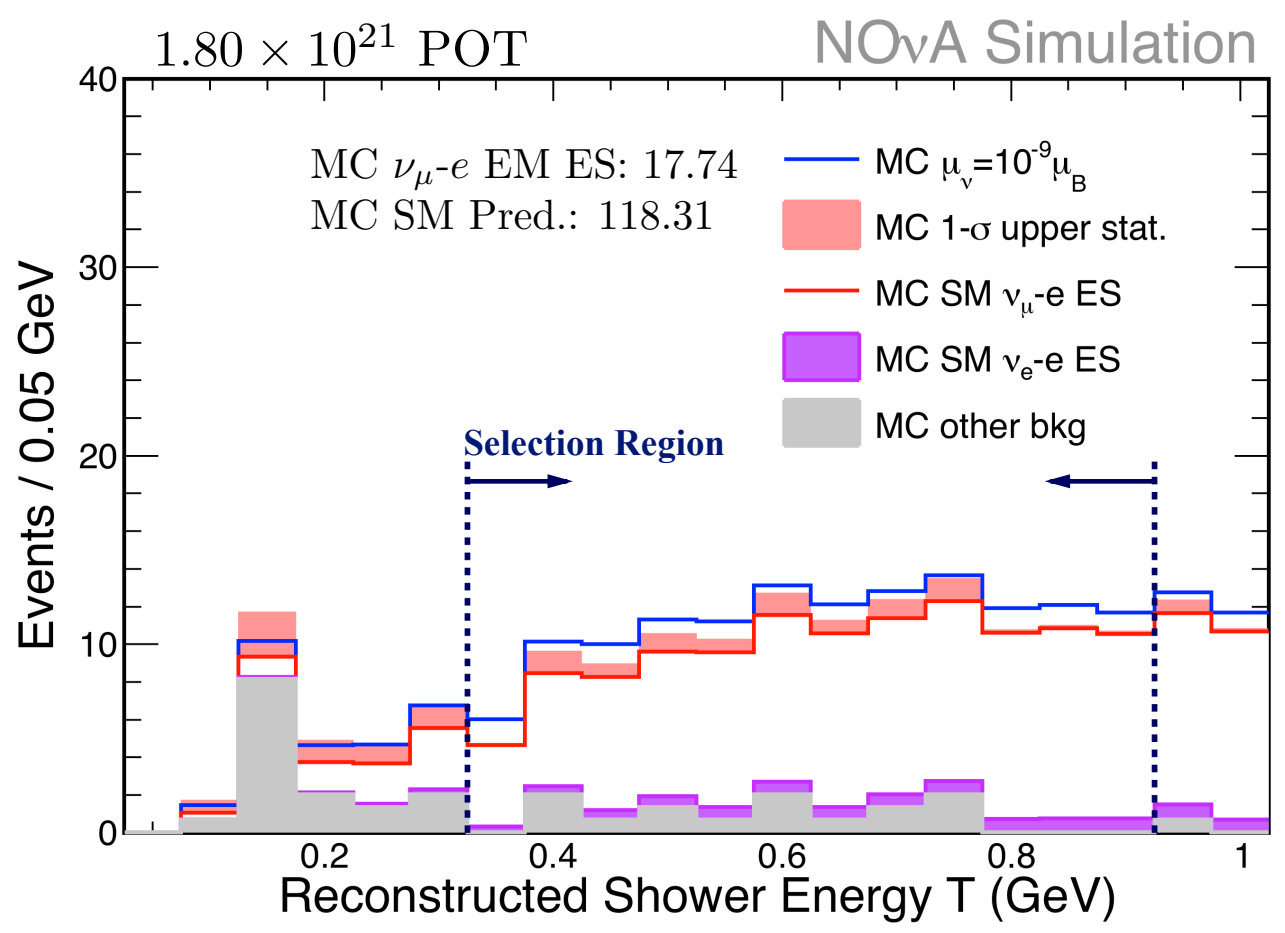

Figure 5.1. Monte Carlo components for the $1.8 \times 10^{21}$ POT dataset. In this plot, all the $\mathrm{MC}$ components are stacked together, so that the area under the red solid line indicates the Standard Model prediction while the area under the blue solid line represents the sum of $\nu_{\mu}$-e magnetic scattering and SM background. The rest of the plots in this chapter follow the same notations here.

We estimate the uncertainties in the low recoil energy cutoff $T$ and the kinematic feature selection $T \theta^{2}$ by calculating the difference of the event number in the region including the selection region plus the most adjacent bin to the nominal selection region. For the threshold of reconstruction electron recoil energy $T$, this ratio represents the uncertainty from the hits clustering method, which originates from the calorimetric slicer4D algorithm [24] that we introduced in Chap. 3. We use the histogram shown in Fig. 5.1 to calculate the systematic uncertainty in event rates. Thus, the systematic uncertainty of this low reconstruction energy cutoff $\Delta_{T}$ is given by

$$
\Delta_{T}=\frac{N_{\text {bins }(6-18)}-N_{\text {bins }(7-18)}}{N_{\text {bins }(7-18)}} .
$$


Thus, for $\mu_{\nu}=10^{-9} \mu_{B}$ resulted muon neutrino on electron magnetic scattering events, we have this systematic uncertainty in event yield $\Delta_{T}(N M M)$ given by

$$
\Delta_{T}(N M M)=\frac{1.22}{17.74}=6.8 \% .
$$

And, for the total Standard Model prediction, we have

$$
\Delta_{T}(S M)=\frac{5.54}{118.31}=4.6 \% .
$$

Therefore, we found the uncertainties in the low recoil energy cutoff for both signal and Standard Model background in MC samples.

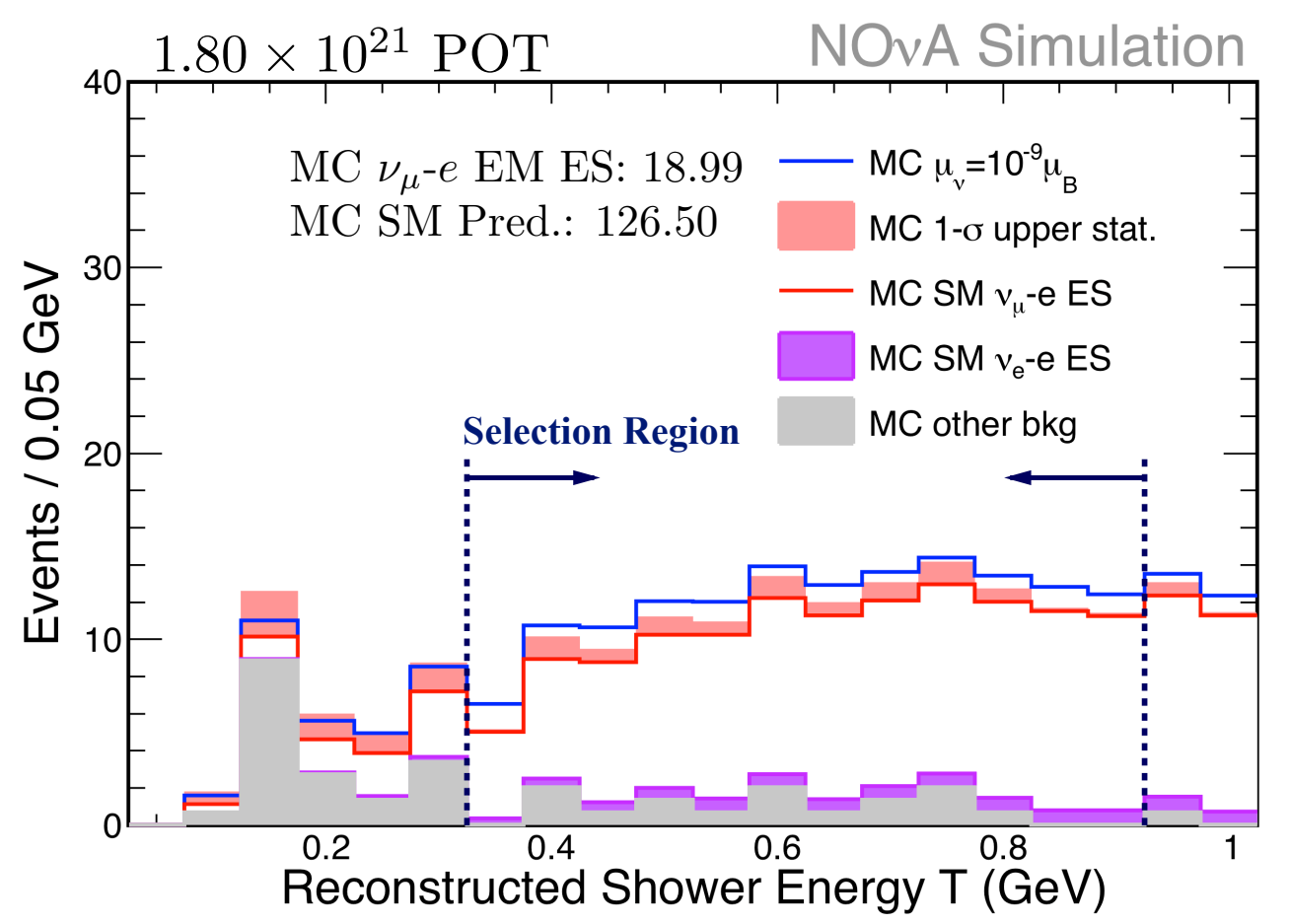

Figure 5.2. Systematic shift in events rate caused by a one-bin shift in the $T \theta^{2}$ cut. We loosen the $T \theta^{2}$ cut by one bin so that $T \theta^{2}<0.0035 \mathrm{GeV} \times \operatorname{Rad}^{2}$ in this plot.

We now focus on analyzing the $T \theta^{2}$ distribution for $T>1 \mathrm{GeV}$ as shown in Fig. 4.5, we can view the $T \theta^{2}$ distribution as a result of the effects that composed of the beam direction 
and the angular solution from the fuzzyK algorithm [32], plus a set of inclusive systematic uncertainties including the PID selections [88]. A practical challenge is that the method of handling systematic uncertainties that propagate through a neural network are not settled yet in experimental high energy physics. Therefore we recall the $T \theta^{2}<0.003 \mathrm{GeV} \times \operatorname{Rad}^{2}$ selection and loosen it by one bin $T \theta^{2}<0.0035 \mathrm{GeV} \times \operatorname{Rad}^{2}$ in Fig. 5.2 to estimate the range of selection shift. The systematic error $\Delta_{T \theta^{2}}$ is given by

$$
\Delta_{T \theta^{2}}=\frac{N\left(T \theta^{2}<0.0035\right)-N\left(T \theta^{2}<0.003\right)}{N\left(T \theta^{2}<0.003\right)} .
$$

Here $\mathrm{N}$ is the number of events in each selection region. Thus, for this type of uncertainty of muon neutrino on electron magnetic scattering, we have

$$
\Delta_{T \theta^{2}}(N M M)=\frac{18.99-17.74}{17.74}=7.1 \%
$$

and for the SM prediction, we have

$$
\Delta_{T \theta^{2}}(S M)=\frac{126.50-118.31}{118.31}=6.9 \%
$$

By now, we have estimated the systematic uncertainties in the signal selection region caused by selection criteria. Then we estimate the systematic shifts caused by Monte Carlo simulations. We discuss the effects from light yield modeling, neutrino flux simulations and cross section precisions in turn.

The NOvA experiment uses liquid scintillator for light generation. The light yield (LY) has an non-linear relation to the energy loss $d E / d x$ by the passing charged particle in the detector [38]. This empirical relation is described by the Birks-Chou formula in Eq. 5.7.

$$
L Y \propto \frac{\frac{d E}{d x}}{1+k_{B} \frac{d E}{d x}+k_{C}\left(\frac{d E}{d x}\right)^{2}}
$$

Birks-Chou constants $k_{B}$ and $k_{C}$ affect at least two consequence, one is the shift in energy scale, and the other is the shift in the $\mathrm{dE} / \mathrm{dx}$ that causing the PID selection to be slightly 
different from the nominal case. We use the scintillation modeling as the NOvA second oscillation analysis [98]. The nominal MC use $k_{B}=0.04 \mathrm{~cm} / \mathrm{MeV}$ and $k_{C}=-0.0005 \mathrm{~cm}^{2} / \mathrm{MeV}^{2}$ in the standard MC simulation [99].

To estimate uncertainty from scintillation modeling, We then apply different values of $k_{B}=0.02 \mathrm{~cm} / \mathrm{MeV}$ and $k_{C}=0.0 \mathrm{~cm}^{2} / \mathrm{MeV}^{2}$ into the $\mathrm{MC}$ simulation [99] and reconstruction chains to generate the so-called "Birks-B" sample and compare the selected events in this Birks-B sample to the standard MC as a systematic shift in the events number in the signal selection region.

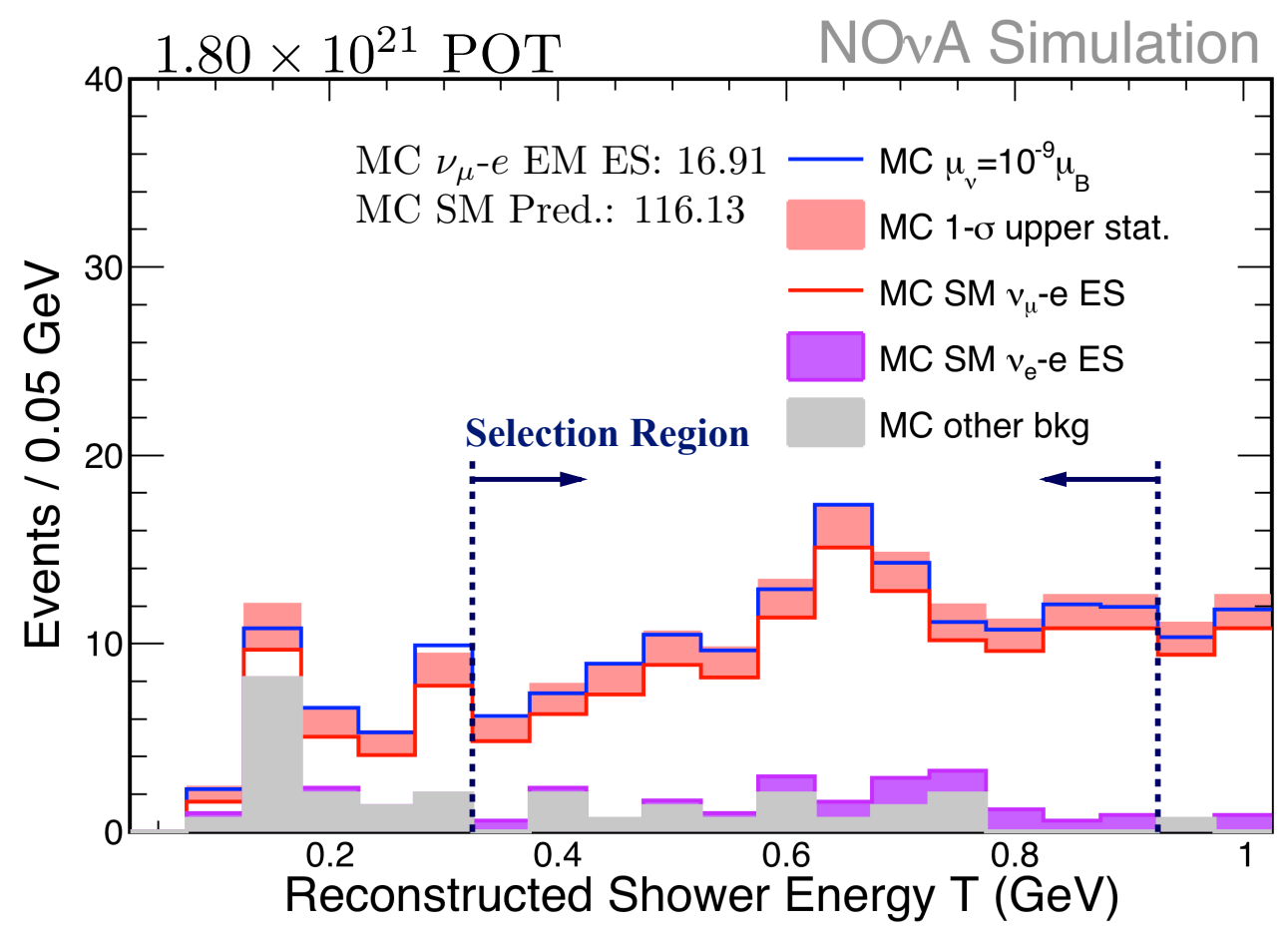

Figure 5.3. Replace the $\nu$-e nominal scattering files with the one with Birks-B constants sample as an estimation of systematic uncertainty in scintillation modeling. Scaled to $1.8 \times$ $10^{21}$ POT.

Since $\nu_{\mu}$ induced magnetic scattering on electrons is sensitive to the region with lower electron recoil energy, as the numerical results shown in Fig 5.3, the systematic error is about 3 times as large as the systematic uncertainty from scintillation modeling in the SM Monte 
Carlo. The systematic uncertainty in event rate caused by scintillation model is given by

$$
\Delta_{\text {Birks }}=\frac{\left|N_{\text {Birks-B }}-N_{\text {nomi }}\right|}{N_{\text {nomi }}} .
$$

Thus, for $\nu_{\mu}$-e magnetic scattering, we have

$$
\Delta_{\text {Birks }}(N M M)=\frac{16.91-17.74}{17.74}=4.6 \%,
$$

and for the SM prediction, we have

$$
\Delta_{\text {Birks }}(S M)=\frac{116.13-118.31}{118.31}=1.8 \% .
$$

The uncertainty in the event rate due to the neutrino flux $\Phi\left(E_{\nu}\right)$ decayed from parent $\pi^{+}$meson decays were estimated by shifting each component in the beam simulation as summarized in Table 4.4. The shift value of each parameter was chosen by the maximum possible physical range.

The $K^{+}$decayed beam also takes a small amount. We see the hint in the neutrinoelectron $T$ distribution that the $K^{+}$simulation might not a hundred percent agrees with the data. The $K^{+}$decayed beam reweight in NOvA second analysis is about $16 \%$ [2], we also include this uncertainty for completeness. Since the muon neutrinos from parent $K^{+}$decays only take a few percent among the total neutrinos, we are safe to reweight twenty percent of the neutrino flux $\Phi\left(E_{\nu}\right)$ from parent $K^{+}$to estimate the uncertainty. 

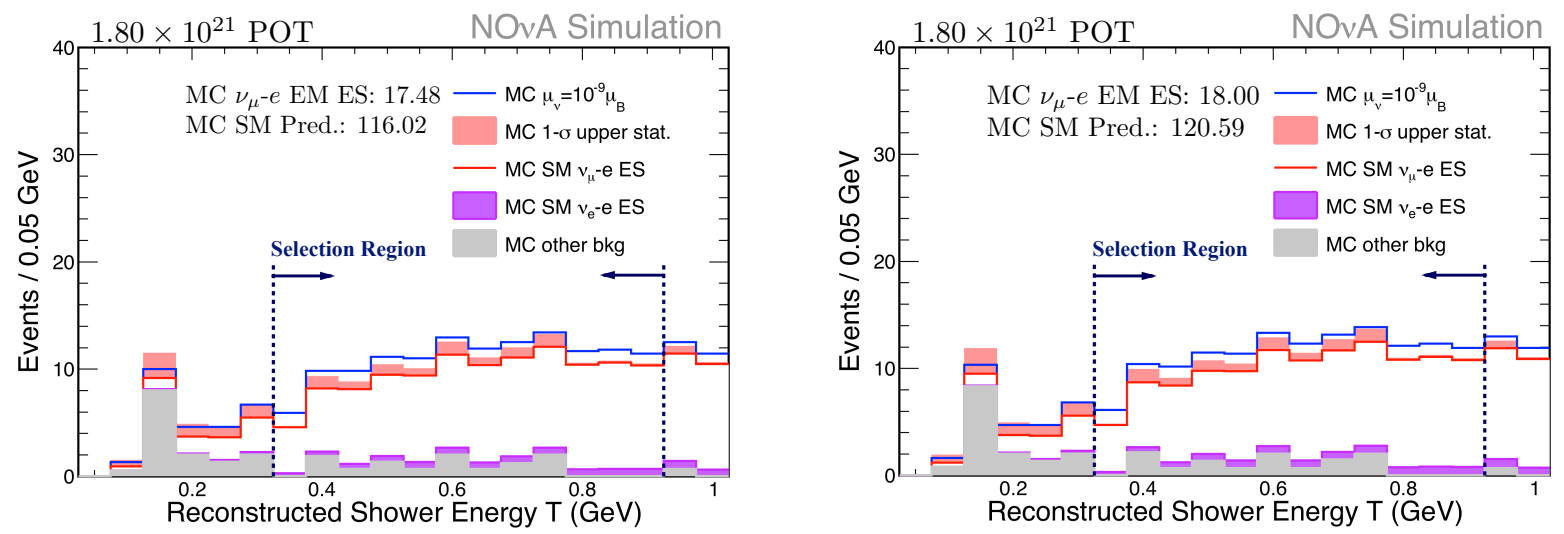

Figure 5.4. Tuning the neutrino flux from parent $K^{+}$decays produced in the NuMI target. The left plot corresponds to a $20 \%$ decrease to the nominal $K^{+}$simulation, while the right plot is increasing $20 \%$.

According to Fig. 5.4, we calculate the systematic uncertainty by

$$
\Delta_{K^{+}}=\frac{\left|N\left(K^{+}+20 \%\right)-N\left(K^{+}-20 \%\right)\right|}{2 N(\text { nomi })} .
$$

So that for $\nu_{\mu}$-e magnetic scattering, we have

$$
\Delta_{K^{+}}(N M M)=\frac{18.00-17.48}{17.74 \times 2}=1.5 \%,
$$

and for the SM prediction, we have

$$
\Delta_{K^{+}}(S M)=\frac{120.59-116.02}{118.31 \times 2}=1.9 \% .
$$

The uncertainty of implementation of $\nu$-e scattering in GENIE package [14] is one percent. Remember that when we select the $\nu$-e scattering events, the dominant background for $\nu$-e scattering is the neutral current coherent scattering events. Then we use the uncertainty from neutral current coherent analysis in the near detector (16.7\%) [51] and cross-section tuning ( 25\%) [27] as the total cross-section fluctuation on all the other non $\nu$-e component background to get the quadrature $(30 \%)$ as the hadronic cross-section uncertainty. 
Thus we summarize the total systematic uncertainty in the number of events that passing our selections in Tab. 5.1.

\begin{tabular}{lcc}
\hline Source of systematic shifts in $\mathrm{MC}$ & $\nu_{\mu}-e \mathrm{EM}\left(10^{-9} \mu_{B}\right) \mathrm{ES}$ & Total SM \\
\hline Low T cutoff & $6.8 \%$ & $4.6 \%$ \\
$\mathrm{~T} \theta^{2}$ cut & $7.1 \%$ & $6.9 \%$ \\
Birks B scintillation modeling & $4.6 \%$ & $1.8 \%$ \\
Parent $\pi^{+}$in FLUKA & $5.9 \%$ & $5.9 \%$ \\
Parent $K^{+}( \pm 20 \%)$ & $1.5 \%$ & $1.9 \%$ \\
GENIE $\nu$ - $e$ x-sec & $1.0 \%$ & $1.0 \%$ \\
Other hadronic BG x-sec $(\sim \pm 30 \%)$ & $\mathrm{N} / \mathrm{A}$ & $2.7 \%$ \\
\hline Quadrature systematic error & $12.5 \%$ & $10.9 \%$ \\
\hline
\end{tabular}

Table 5.1. Summary of the systematic uncertainties for number of selected events. The first column lists the source of each systematic shift, the second column lists the change of number of selected events for $\nu_{\mu}$-e magnetic scattering with $\mu_{\nu_{\mu}}=10^{-9} \mu_{B}$ and the third column lists the change in the Standard Model background.

The total systematic uncertainty in events rate of $10.9 \%$ is close to the assumption we made $(10 \%)$ when determining the signal region for $\nu_{\mu}$ induced magnetic scattering, which indicated that the statistical counting is sufficient to include the $10.9 \%$ systematic error on standard model Monte Carlo. We now look into the data and make a comparison plot of the data and the Monte Carlo in the $T$ selection region as shown in Fig. 5.5. 


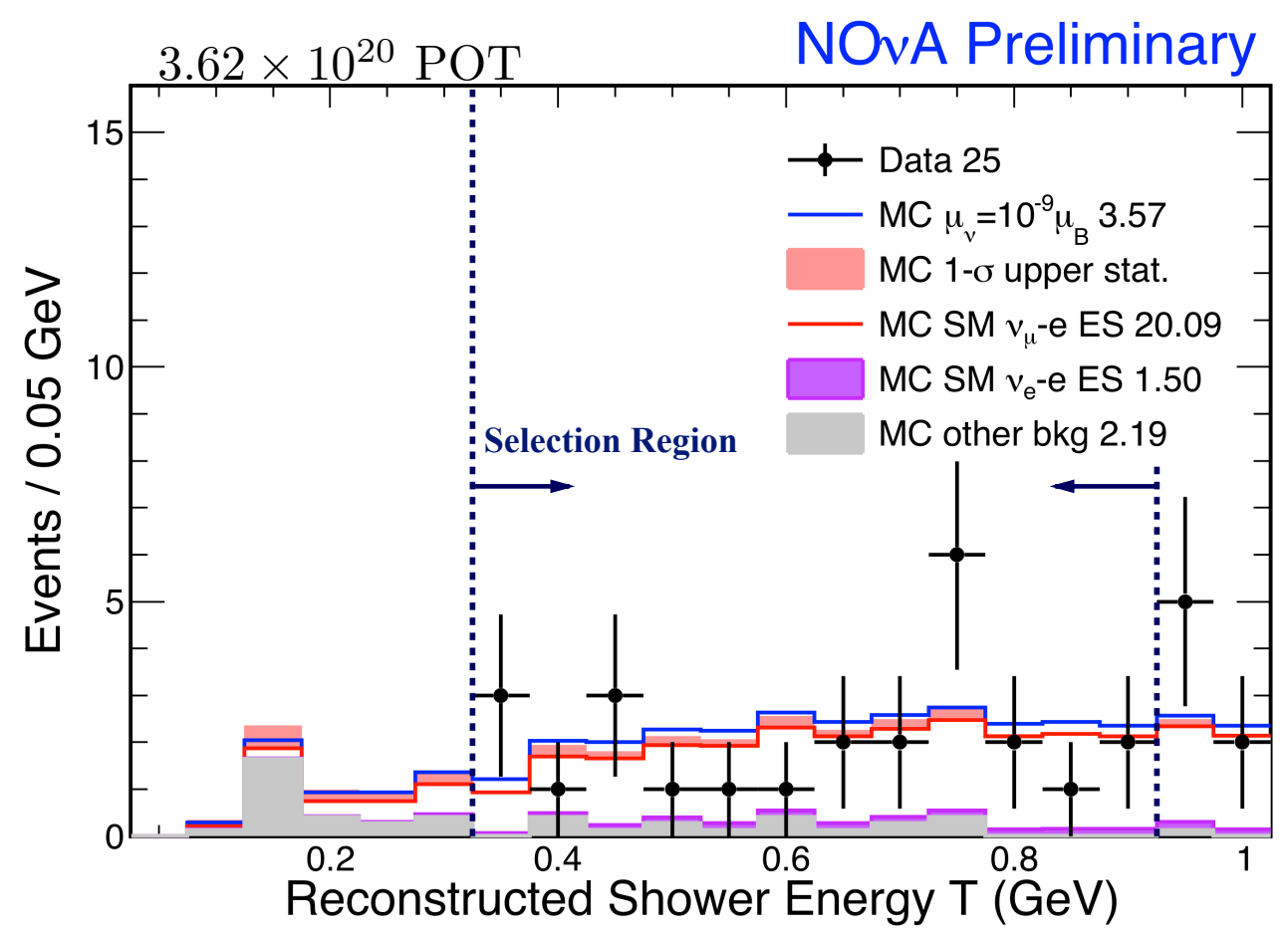

Figure 5.5. Data/MC comparison for searching for neutrino magnetic moment by using $3.62 \times 10^{20} \mathrm{POT}$ in NOvA near detector. In the signal selection region, we see 25 events in data while 23.78 events in the MC SM prediction.

The number of MC $\nu$-e scattering events in Fig. 5.5 are scaled down from the one corresponding to $6.74 \times 10^{23} \mathrm{POT}$. Thus the statistical uncertainty in $\nu$-e $\mathrm{MC}$ can be neglected.

The question now becomes, given the estimated systematic range in the events rate in Monte Carlo and the statistical fluctuation in data, what is the upper limit on the average signal rate for a given confidence level in the signal selection region? We address this issue in the next section. 


\subsection{Statistical Methods for the Upper Limit}

In general, if the signal significance is higher than $5 \sigma$, then we announce a discovery. If the significance is about $3 \sigma$, then we make an observation and provide the value of the measurement. In our case, the significance is less than two sigma, so we report an upper limit at 90\% confidence level (C.L.).

Given the $3.62 \times 10^{20} \mathrm{POT}$, we consider the data to $\mathrm{MC}$ comparison as a simple number of events counting experiment. Once we collect sufficient data, more advanced tools will be applied in the data analysis.

The concepts and techniques of setting an upper limit to a rare process are essential for exotic searches beyond the Standard Model physics. There are currently two mainstream philosophies for analyzing this type of issue: Bayesian and Frequentist.

In the Bayesian approach, we assume that the neutrino magnetic moment is a random variable, and that it has a prior probability distribution for its value. Suppose $s$ is the number of signal events in the selection region, and $\alpha$ is the significance level, then $1-\alpha$ represents the confidence level. After the measurement, we report an upper limit at $(1-\alpha)$ C.L. by finding a value $s_{u p}$ for the posterior probability given by Eq. 5.14.

$$
\operatorname{Prob}\left(s \geq s_{u p}\right)=\alpha \times \operatorname{Prob}(s \geq 0)
$$

This method was used in the analysis of solar electron neutrino data in the Super-Kamiokande (SK) experiment [69]. The SK data is sufficient for spectrum fitting, so that a $\Delta \chi^{2}$ (described in Ref. [70]) is calculated instead. The 90\% C.L. upper limit on solar neutrino magnetic moment [70] is calculated from

$$
\operatorname{Prob}\left(\Delta \chi^{2}\left(s \geq s_{u p}\right)\right)=0.1 \times \operatorname{Prob}\left(\Delta \chi^{2}(s \geq 0)\right)
$$

In the Frequentist approach, the actual muon neutrino magnetic moment is an unknown constant. We define the upper limit of the number of $\nu_{\mu}-e$ EM scattering events $s_{u p}$ so that 
for $\mathrm{a}\left(s_{u p}+b\right)$ distribution, where $\mathrm{b}$ is the number of SM background, the probabilities for $n \leq n_{\text {obs }}$ adds up to a value $\alpha$

$$
\sum_{n=0}^{n_{\text {obs }}} \operatorname{Prob}\left(n \mid s_{\text {up }}, b\right)=\alpha
$$

Since the NOvA near detector is close to the neutrino source, we ignore the short baseline oscillations for now. For a total of 25 events, we consider the $s+b$ distribution as a Poisson procedure. We report a one-sided confidence interval, by counting the area of this $s+b$ distribution on one side from the number of observed data. We then find a value for $s_{u p}$ that satisfy the Eq. 5.16 for fixed $b=23.78$ and $n_{o b s}=25$ as shown in Fig. 5.5. Ref. [41] gives the full details of the statistical technique.

Also, by this definition, the nominal upper limit of a signal process is actually the upper limit of its mean value of the $s_{u p}+b$ Poisson distribution, also called median upper limit for symmetric distribution [43]. Most of the Frequentist methods report the systematic uncertainty instead of counting it into the $s+b$ distribution.

Both the Bayesian and Frequentist methods can be applied in the rare process searches. However, it is more complicated and obscure in the Bayesian approach when people try to interpret different types of the prior distributions into the physics meanings behind them. In contrast, the Frequentist method simply assumes that the magnetic moment of neutrino is an unknown constant and thus it is easier for both theorists and experimentalists to apply the results. Hence, we use several Frequentist methods in this thesis.

In the following subsections, three classical Frequentist methods are applied, and the systematic uncertainty for each is discussed. Firstly, we use the Chi-Squared quantile method to calculate the upper limit and its systematic uncertainty. Secondly, we use the FeldmanCousins method to check the physical boundary and cross check the result. Finally, we use the profile likelihood method to calculate the upper limit with and without the systematic uncertainty in the number of SM events to see the systematic effect on the final results. 


\subsubsection{Chi-Squared Quantile Method}

This subsection follows closely the methods discussed in Ref. [42, 106]. We are setting a one-sided upper limit for the mean value of an unknown constant s. In our case, s is the number of $\nu_{\mu}$-e EM elastic scattering (ES) events. The Poisson distribution gives the probability for observing a total number of $n$ events with a well-known Standard Model background $b$ and an unknown signal $s$.

$$
\operatorname{Prob}(n \mid s, b)=\frac{(s+b)^{n} e^{-(s+b)}}{n !}
$$

Following a mathematical transformation [42], we have the following relation between a Poisson distribution and a $\chi^{2}$ distribution

$$
\sum_{n=0}^{i} \operatorname{Prob}(n \mid k)=\int_{2 k}^{\infty} f_{\chi^{2}}(y ; 2(i+1)) d y .
$$

Here, the function $f_{\chi^{2}}(y ; 2(i+1))$ is the probability density function of a $\chi^{2}$ distribution with $2(i+1)$ degrees of freedom, $k=s+b$ in our case, and $i=n_{\text {obs }}$, the number of observed events.

After the above integration, we get a relation with its cumulative distribution,

$$
\sum_{n=0}^{i} \operatorname{Prob}(n \mid k)=1-F_{\chi^{2}}(2 k ; 2(i+1)) .
$$

We then substitute this relation into Eq. 5.16, and find the following relations between the significance level $\alpha$ and the upper limit $s_{u p}$ corresponding to the $(1-\alpha)$ C.L.:

$$
\begin{aligned}
& \alpha=1-F_{\chi^{2}}\left(2\left(s_{u p}+b\right) ; 2\left(n_{o b s}+1\right)\right), \\
& 2\left(s_{u p}+b\right)=F_{\chi^{2}}^{-1}\left(1-\alpha ; 2\left(n_{o b s}+1\right)\right), \\
& s_{u p}=\frac{1}{2} F_{\chi^{2}}^{-1}\left(1-\alpha ; 2\left(n_{o b s}+1\right)\right)-b .
\end{aligned}
$$


For our first analysis of muon neutrino magnetic moment, we observed 25 events with 23.78 events expected from standard model background. The event rate in the selection recoil energy $T$ range $R(T)$ for magnetic scattering has the following relationship derived from theoretical $\nu_{\mu}-e$ cross-section described in chapter two,

$$
R(T) \propto \frac{\mu_{\nu}^{2}}{\mu_{B}^{2}} \frac{\pi \alpha_{E M}^{2}}{m_{e}^{2}} \int_{T_{1}}^{T_{2}}\left(\frac{1}{T}-\frac{1}{E_{\nu}}\right) d T .
$$

For a fixed integral of recoil energy $T$ range, and knowing from MC that $\mu_{\nu}=10^{-9} \mu_{B}$ corresponds to 3.57 scattering events, we can convert the number of events passing our selection cuts into a value for the square of the muon neutrino magnetic moment by using the relation

$$
\frac{\mu_{\nu}^{2}}{\left(10^{-9} \mu_{B}\right)^{2}}=\frac{R(T)}{3.57} .
$$

Using the Eq. 5.22 and a ROOT built-in function "TMath::ChisquareQuantile", we can perform this calculation. Therefore, at $1-\alpha=90.0 \%$ conference level, $s_{u p}=8.93$ events, corresponding to $1.58 \times 10^{-9} \mu_{B}$ from Eq. 5.24. Since Eq. 5.22 shows that the $s_{u p}$ has a linear relationship with background $b$, it is convenient to apply the systematic uncertainty found in Table. 5.1, thus $b=23.78 \pm 2.59$. Then we get $s_{u p}=8.93 \pm 2.59$, note the error range on $\mathrm{b}$ and

sup are the same for this specific method. This value corresponds to $\mu_{\nu}=1.58_{-0.25}^{+0.22} \times 10^{-9} \mu_{B}$ by Eq. 5.24 .

\subsubsection{Feldman-Cousins Method}

The Chi-Squared Quantile is appropriate when the total observed event number is large [106]. Suppose we observe six events in our signal region with 23.78 events predicted by standard model simulation. Then the Chi-Squared Quantile method yields $s_{u p}=-13.25$ events, a negative value which is impossible in reality since the rare signal always has $s \geq 0$.

To avoid such a non-physical situation, G. Feldman and R. Cousins developed the unified approach by using likelihood ratio ordering [53]. The algorithm is described in pseudocode in Fig. 5.6. One has to define a range of signal parameters as a set of continuous real number 
$\left[0, s_{\max }\right]$, and for each $\mathrm{s}$ in this range, assign a set of possible integers as observed events from 0 to $N_{\max }$. We then compute a set of likelihood ratios $R(N)$, and rank them from the largest to the lowest, using

$$
R(N)=\frac{\operatorname{Prob}(N \mid s)}{\operatorname{Prob}\left(N \mid \mu_{\text {best }}\right)}=\left(\frac{s+b}{\mu_{\text {best }}+b}\right)^{N} e^{\mu_{\text {best }}-s} .
$$

We then sum the probabilities $\operatorname{Prob}(N \mid s, b)$ for a certain $s$, until the sum no less than the corresponding confidence level $(1-\alpha)$,

$$
\sum \operatorname{Prob}\left(N_{(R)} \mid s, b\right) \geq 1-\alpha .
$$

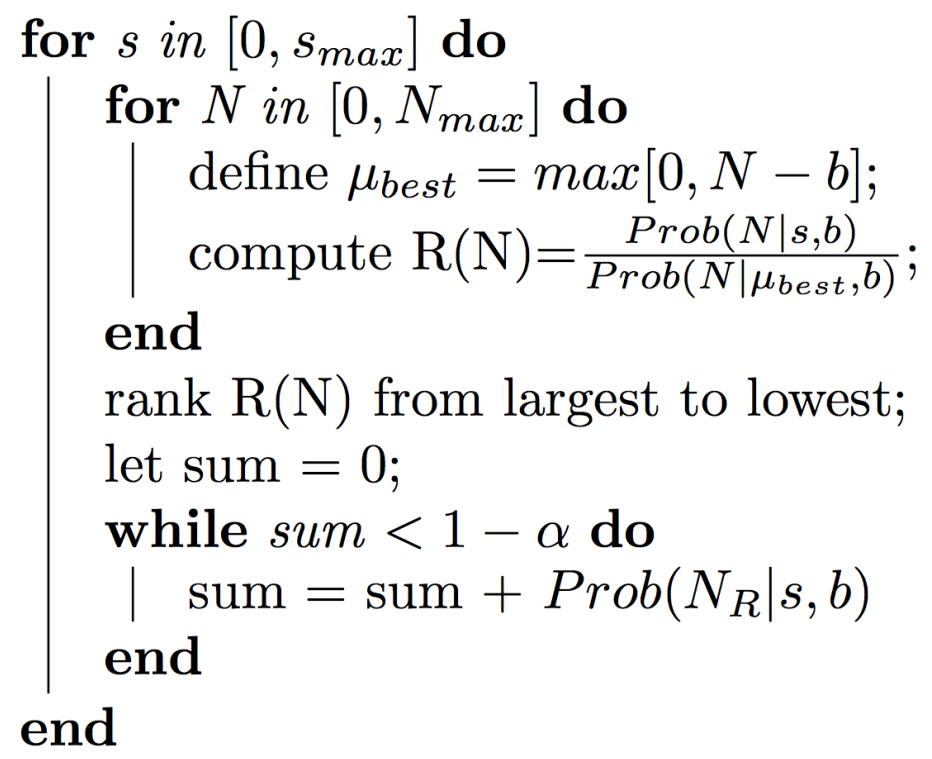

Figure 5.6. Pseudocode for the Feldman-Cousins method.

At the end of this method, each s is associated with a set of numbers of possible observed events $N$ [83]. In other words, given a fixed number of observed events $\mathrm{N}$, we also get a series of s from $s_{\min }$ to $s_{\max }$ such that the sum of the probabilities associated with the s values no less than $(1-\alpha)$. The largest $\mathrm{s}$ in that series is the upper limit at a given confidence level. 
We let s range from zero to $s_{\max }=10$ and set the interval $\Delta s=0.001$ to optimize the precision, then apply this method on a $3.62 \times 10^{20}$ POT dataset and get the results as shown in Fig. 5.7 and Fig. 5.8. We use a python package called "gammapy.stats" to plot this result [49].

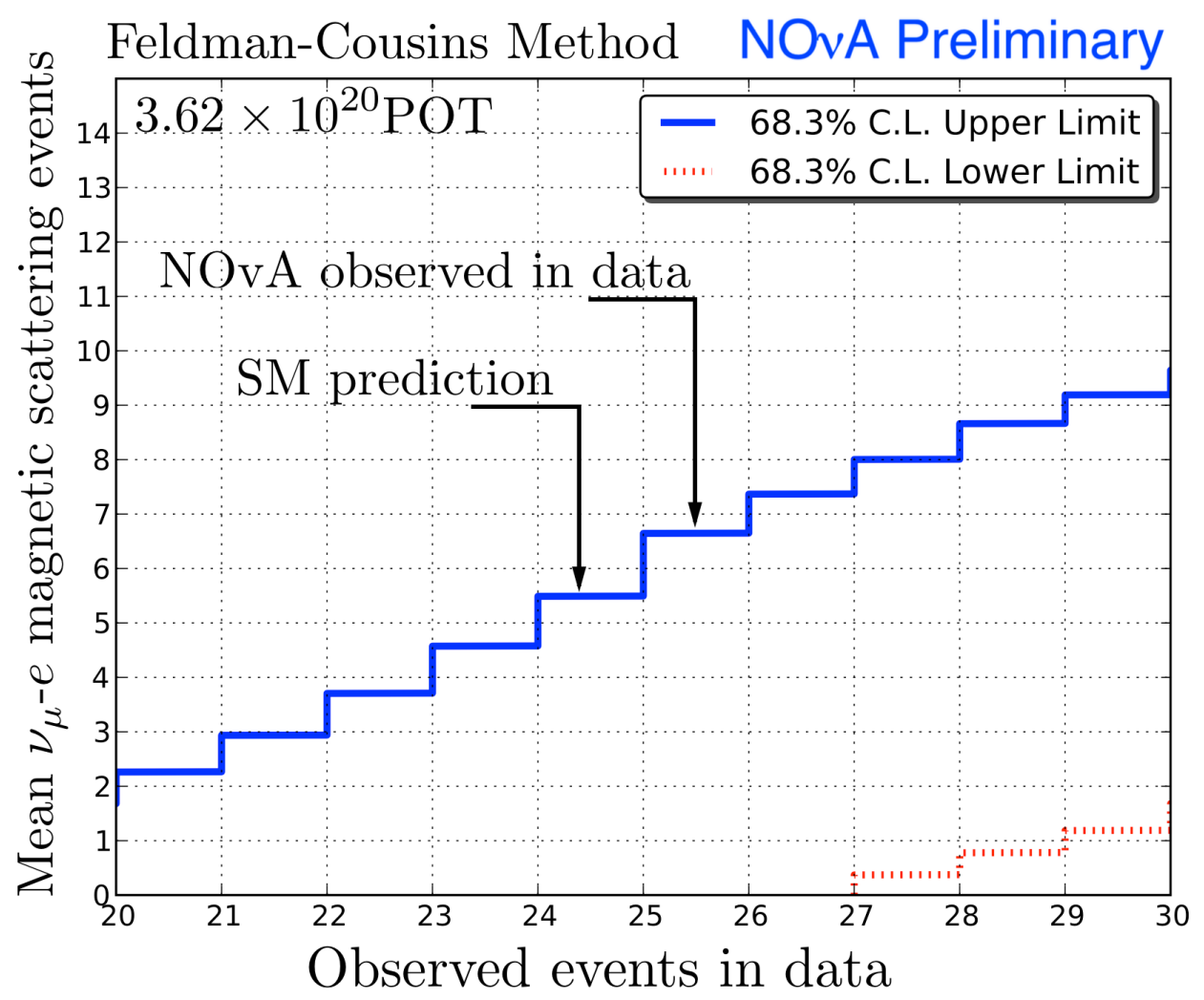

Figure 5.7. Using the Feldman-Cousins Method to calculate upper limit as a function of observed number of events in data for $68.3 \%$ C.L., we also provide the lower limit to see the if there is a non-zero hypothesis.

In Fig. 5.7, we use the Feldman-Cousins Method and display the boundary of statistical limits for $68.3 \%$ confidence level. As shown in this plot, if the number of observed events is no less than 27, then there exists a hint for non-zero magnetic moment of muon neutrino since we get a positive lower limit. However, we only see 25 events in the $3.62 \times 10^{20} P O T$ dataset, which indicates that the result is consistent with the Standard Model prediction. So we see no evidence for new physics yet. 


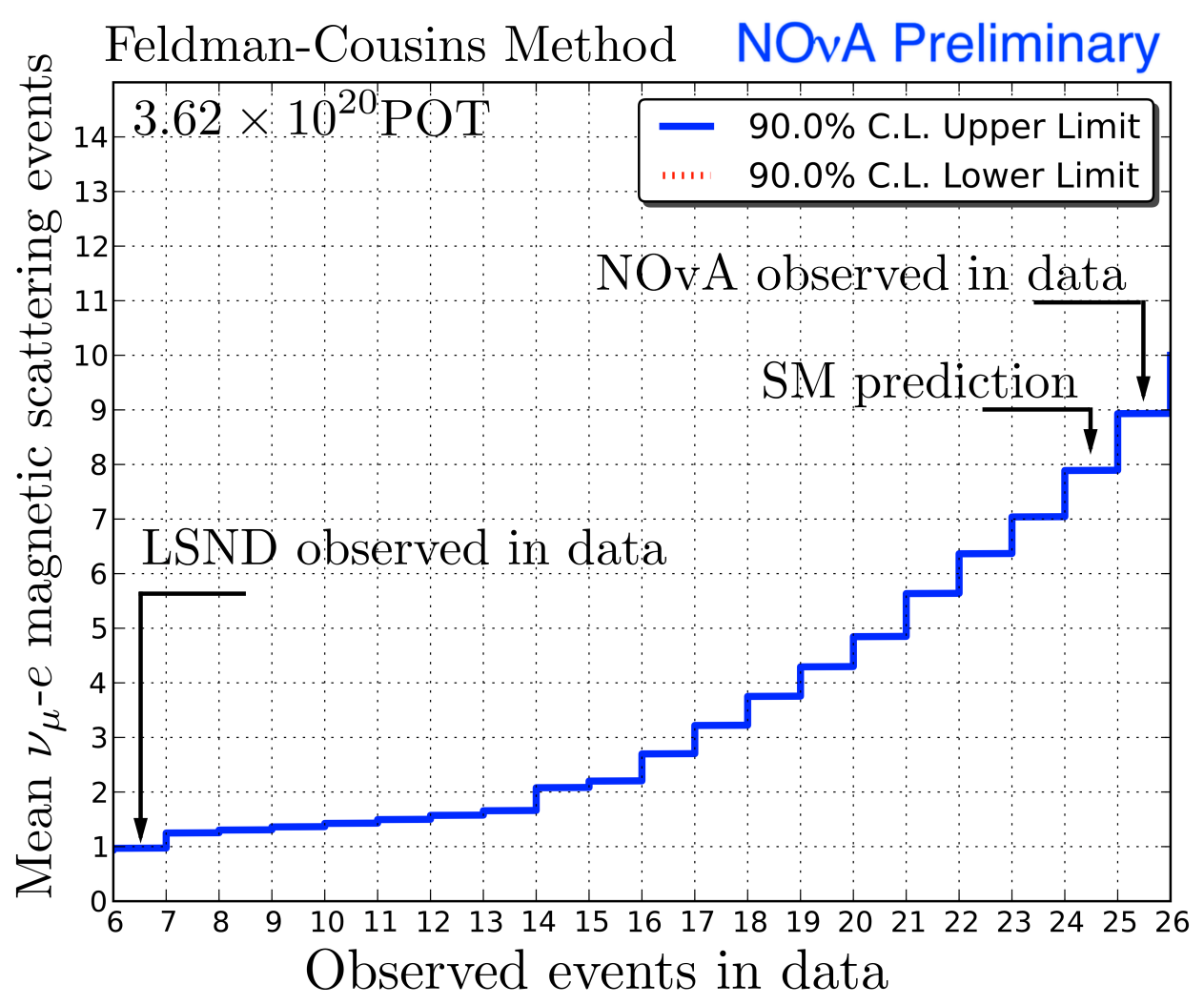

Figure 5.8. Using the Feldman-Cousins Method to calculate upper limit as a function of the observed number of events in data at $90.0 \%$ confidence level. This plot illustrate that even given an occasionally observable much lower than the SM prediction in dataset, the F-C method still give a reasonable coverage for the upper limit of the unknown rare signal.

In Fig. 5.8, we notice that the Feldman-Cousins procedure does solve the problem for the physical boundary when the observed event number approaches zero. In our current dataset, we observed 25 events in data, and Feldman-Cousins method calculates an upper limit of $s_{u p}=8.93$ for $90.0 \%$ confidence level, coincidentally agreeing with the result from the classic $\chi^{2}$ quantile calculation. The Feldman-Cousins Method has to be used under the assumption that the background is well-known, thus we report the systematic uncertainties instead of involving the systematic uncertainty in this Feldman-Cousins approach.

Since the LSND experiment is $\nu_{e}-e$ scattering dominant and $\nu_{\mu}-e$ scattering were considered as background, it is impossible to directly compare the sensitivities from NOvA with the final results from LSND. If we observe six events in the NOvA near detector, the upper limit 
from the Feldman-Cousins Method gives $s_{u p}=0.97$ at $90.0 \%$ confidence level. It corresponds to $\mu_{\nu_{\mu}}=0.52 \times 10^{-9} \mu_{B}$, which is slightly more strict than the LSND result $0.68 \times 10^{-9} \mu_{B}$. In the next subsection, we cross check these results from the Feldman-Cousins approach and the classic $\chi^{2}$ quantile method with systematic shift in $b$ by using the profile likelihood method.

\subsubsection{The Profile Likelihood Method}

Reference [71] introduces a method that deals with the upper limit of a Poisson process over a background of Gaussian distribution. Our case is a number counting problem in one bin so that the profile likelihood $L$ is the probability given by

$$
L(s, b \mid N) \equiv \operatorname{Prob}(N \mid s, b) .
$$

The profile likelihood ratio $\lambda$ is defined as

$$
\lambda \equiv \frac{L(s=0, b \mid N)}{L(s, b \mid N)} .
$$




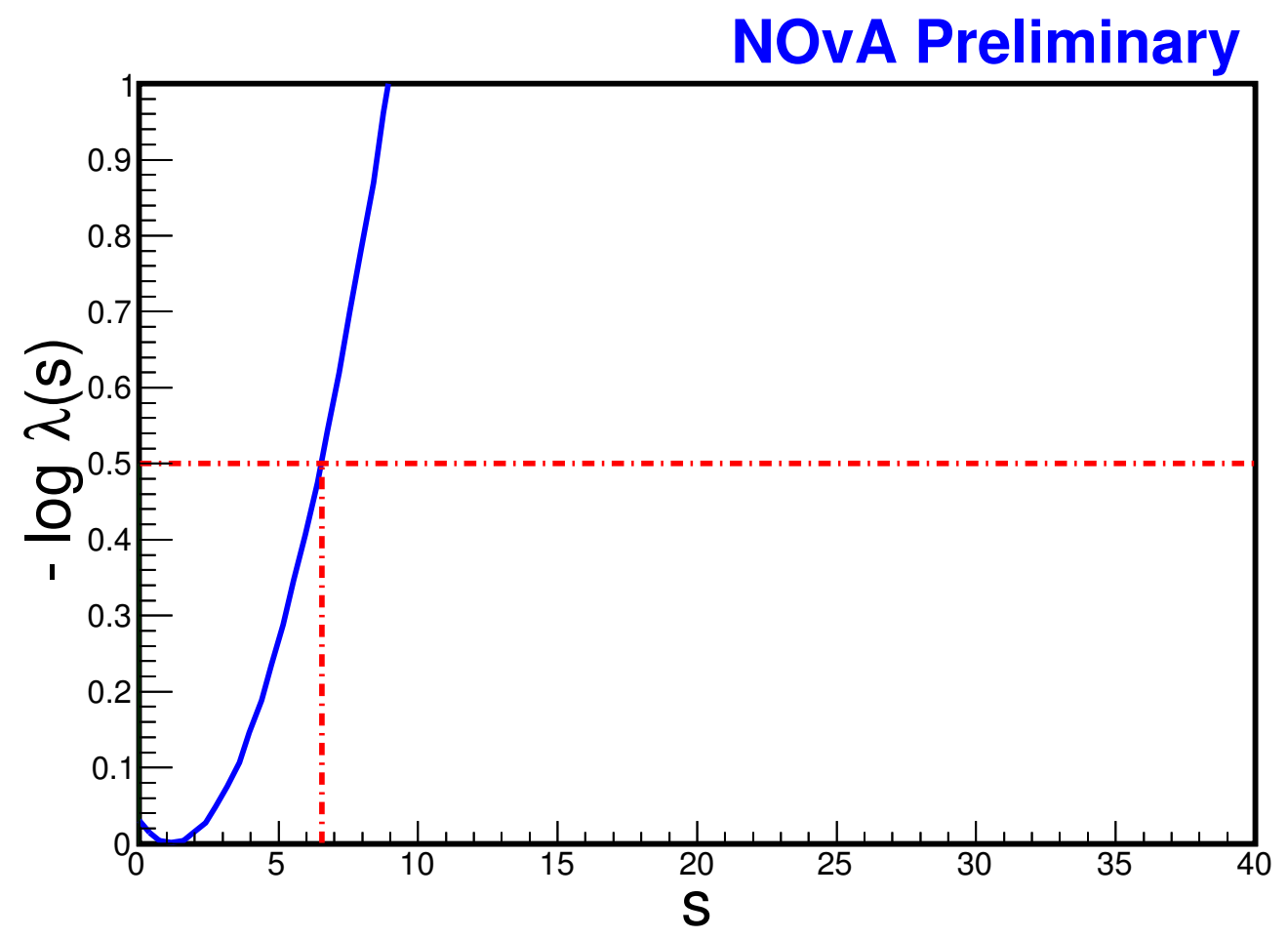

Figure 5.9. Using the profile likelihood ratio function $\lambda$ to estimate an upper limit of $s$ at $68.3 \%$ C.L., with $b=23.78$ and $N=25$.

Thus, according to Wilks' theorem [103], the $\lambda$ function has a mathematical relation with the one degree of freedom $\chi^{2}$ function,

$$
\lambda \approx e^{-\frac{1}{2} \chi_{0}^{2}}
$$

Hence,

$$
-\log \lambda \approx \frac{1}{2} \chi_{0}^{2}
$$

which means that the function $-\log \lambda$ has an distribution of $\frac{1}{2} \chi_{0}^{2}$ approximately with one degree of freedom. 


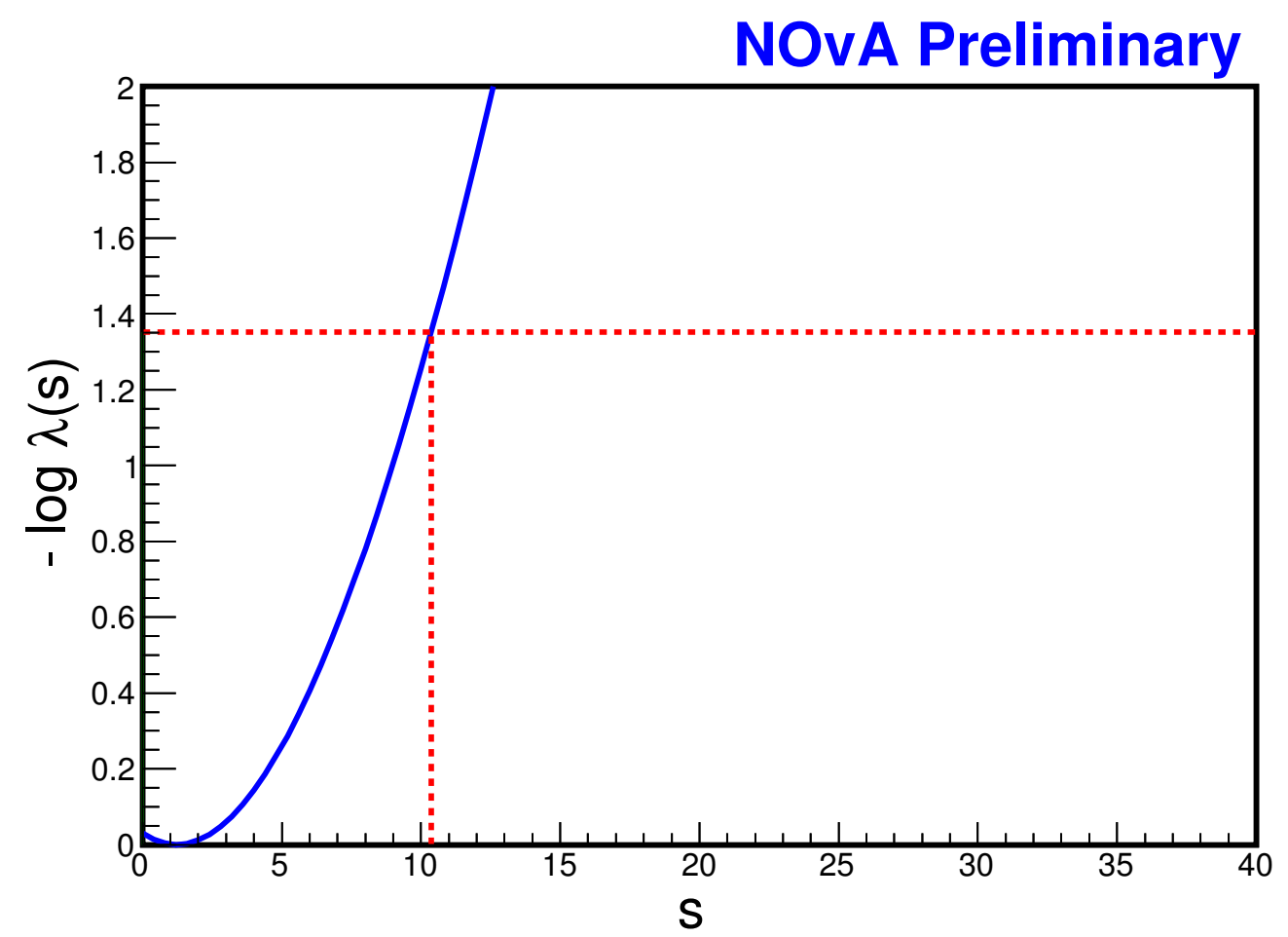

Figure 5.10. Using the profile likelihood ratio function $\lambda$ to estimate an upper limit of $s$ at $90.0 \%$ C.L., with $b=23.78$ and $N=25$.

Figure 5.9 and figure 5.10 illustrate the process to calculate the $s_{u p}$. Start at the minimum value of function $-\log \lambda$, which is zero in our case, by finding

$$
\frac{d(-\log \lambda(s))}{d s}=0 .
$$

In the number counting case when the total event number in data $N$ is larger than the SM background prediction $b$, the zero point location is at $s=N-b$. Then we move the coordinates on the $-\log \lambda$ function to the right-hand side of the minimum value to find the points where the value of this function increases by the corresponding confidence interval of the $\frac{1}{2} \chi_{0}^{2}$ distribution with one degree of freedom. Note that this profile likelihood method is valid when $s+b$ is sufficiently large [41], so we use it merely to cross check the upper limits. 


\begin{tabular}{llllll}
\hline Changes in $-\log \lambda(s)$ & & & & & \\
\hline Confidence level (\%) & 68.3 & 80.0 & 90.0 & 95.4 & 99.7 \\
\hline$\frac{1}{2} \Delta \chi_{0}^{2}($ ndf $=1)$ & 0.50 & 0.82 & 1.35 & 2.00 & 4.50 \\
\hline
\end{tabular}

Table 5.2. The table for $\frac{1}{2} \Delta \chi_{0}^{2}$ values.

For the numerical examples shown in Table. 5.2, we find that $\Delta \chi_{0}^{2}=1.0$ at $68.3 \%$ C.L., and then $-\log \lambda(s)=0.5$. The corresponding value of $s_{u p}$ is then 6.57 events. For $90.0 \%$ C.L., $\Delta \chi_{0}^{2}=2.71$, so that $-\log \lambda(s)=1.36$ and $s_{u p}=10.38$. As a result, we find the $1.36 \times 10^{-9} \mu_{B}$ as the $68.3 \%$ C.L. median upper limit, which agrees with the result from the Feldman-Cousins method without systematic shift. And the 90.0\% C.L. median upper limit is $1.70 \times 10^{-9} \mu_{B}$, slightly higher than the Feldman-Cousins method.

We consider the systematic error in the Standard Model prediction $b$ as the $1 \sigma$ Gaussian deviation from a Gaussian background. We then apply the number of events in data $N$, Standard Model background $b$ and the systematic uncertainty $\delta b$ in the TRolke package introduced in Ref. [71] to calculate $s_{u p}$. We therefore get $1.41 \times 10^{-9} \mu_{B}$ for the upper limit at $68.3 \%$ C.L. and $1.77 \times 10^{-9} \mu_{B}$ for the $90.0 \%$ C.L. By using this comparison between including systematic uncertainty and not including systematic uncertainty, we demonstrated that the systematic shift in Monte Carlo causes a relatively small effect compare to the statistical uncertainty in the limited dataset. 


\subsubsection{Preliminary Result for NOvA 2016}

There is no settled method for experimental neutrino physicists to include systematic uncertainties in limit estimations yet, therefore we report systematic errors separately and assume MC is well-known to set the upper limit in the Feldman-Cousins method. In Table. 5.3, the preliminary results from the different statistical methods are listed. We report $1.58 \times 10^{-9} \mu_{B}$ as the median upper limit at $90.0 \%$ C.L. for NOvA second analysis dataset in 2016 .

\begin{tabular}{lcc}
\hline Methods & Upper limit at $68.3 \%$ C.L. & Upper limit at 90.0\% C.L. \\
\hline$\chi^{2}$ Quantile & $1.11_{-0.40}^{+0.29} \times 10^{-9} \mu_{B}$ & $1.58_{-0.25}^{+0.22} \times 10^{-9} \mu_{B}$ \\
Feldman-Cousins & $1.36 \times 10^{-9} \mu_{B}$ & $1.58 \times 10^{-9} \mu_{B}$ \\
Profile likelihood ratio & $1.36 \times 10^{-9} \mu_{B}$ & $1.70 \times 10^{-9} \mu_{B}$ \\
Profile likelihood ratio with syst. & $1.41 \times 10^{-9} \mu_{B}$ & $1.77 \times 10^{-9} \mu_{B}$ \\
\hline \hline NOvA (2016) & & $1.58 \times 10^{-9} \mu_{B}$ \\
\hline MiniBoone (2008) $[78]$ & & $1.27 \times 10^{-9} \mu_{B}$ \\
\hline
\end{tabular}

Table 5.3. A comparison of the preliminary results from different statistical methods.

In this table, we use three different methods, with different treatments on the systematic uncertainty, to set an upper limit on muon neutrino magnetic moment. In the classic $\chi^{2}$ quantile method, since $s_{u p}$ has a linear relationship with Standard Model background $b$, we can apply the systematic range directly on $b$. For the profile likelihood ratio method, the systematic range is considered to be the $1 \sigma$ deviation from the background.As an estimation, the results are closer to each other in these two methods. These methods give approximately the same results. We noted that the muon neutrino magnetic moment of our result is close to the result from MiniBoone experiment in 2008. 


\subsection{Future Sensitivity for the Full Dataset}

In the next six years, NOvA will accumulate sufficient data to do a more complete analysis. By that time, the uncertainties from neutrino flux are likely to be better well constrained. And the scintillation modeling and the cross sections for hadronic background components will be better addressed than the situation in 2016. For sensitivity estimation purposes, we assume the systematic uncertainties associated with the neutrino flux and the hadronic components are negligible, then optimize $\mathrm{FOM}=s / \sqrt{b}$ to get the new signal selection region as shown in Fig. 5.11.

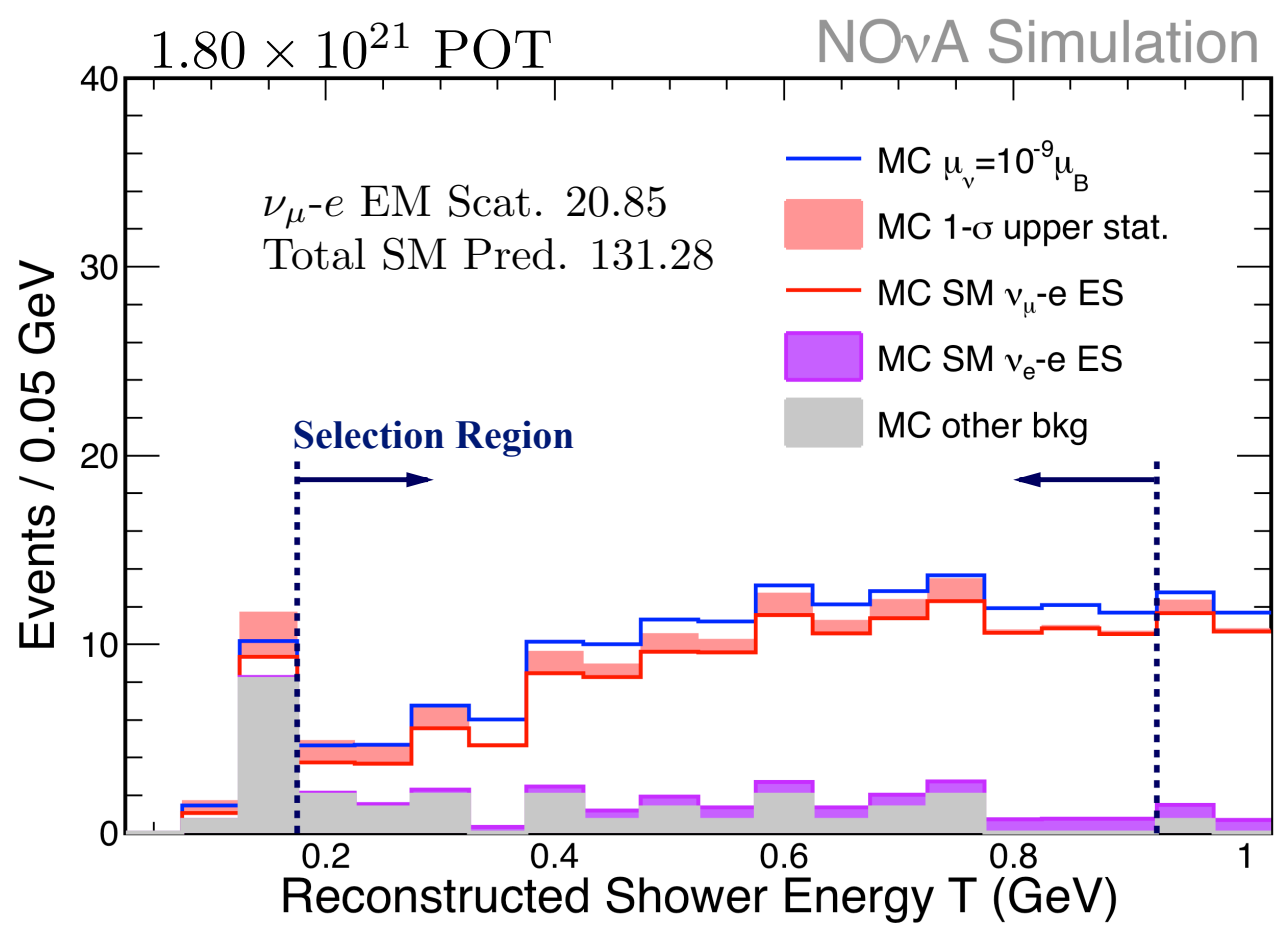

Figure 5.11. The simulated $T$ distribution for $1.8 \times 10^{21}$ POT. For a value of $\mu_{\nu}=10^{-9} \mu_{B}$, we see 21 simulated EM ES events over the Standard Model prediction of 131 events.

The Power-Constrained Limits is a method to study a process that may not exist and can be easier applied compared to other methods [44]. The method starts from finding a maximum likelihood estimator for the signal events and then adding up the contributions 
from the confidence level

$$
s_{u p}=\hat{s}+\Phi^{-1}(1-\alpha) \sigma
$$

Here $\Phi^{-1}$ is the inverse standard Gaussian cumulative distribution. We discuss this method in a number counting case and estimate the sensitivity.

The general formula in Eq. 5.32 is modified in a number counting case, so that we can estimate the sensitivity by

$$
s_{u p}=\max [0, N-b]+\sqrt{N} \Phi^{-1}(1-\alpha) .
$$

Under the assumption that the number of events in data agrees with the Standard Model prediction, Eq. 5.33 gives us the following sensitivity formula

$$
s_{u p}+b-N=\Phi^{-1}(1-\alpha) \sigma
$$

For $90 \%$ C.L. upper limit, $\Phi^{-1}(1-\alpha)=1.28$, and we use $\sigma=\sqrt{N}$ for Gaussian distribution, so that

$$
\hat{s_{u p}}+b-N=1.28 \sqrt{N} \text {. }
$$

Since we are assuming the number of events in data equals to the Standard Model prediction $(N=b)$, so we have

$$
\frac{\hat{s_{u p}}}{\sqrt{b}}=1.28
$$

This is the reason that $s / \sqrt{b}$ is used as an estimation of sensitivity for new physics searches. We first use a large value of muon neutrino magnetic moment $10^{-9} \mu_{B}$, then count the induced EM elastic scattering events. By simulating $1.8 \times 10^{21}$ POT, 21 signal events for $10^{-9} \mu_{B}$ and about 131 events from SM background would pass the selection cuts. Calculating the significance, we have

$$
s / \sqrt{b}=20.85 / \sqrt{131.28}=1.81
$$


Hence, the significance for $10^{-9} \mu_{B}$ corresponds to more than $1.8 \sigma$ as shown in Fig. 5.12. Given a fixed number of observed events 131, the confidence level of $\mu_{\nu}$ no larger than $10^{-9} \mu_{B}$ is $97 \%$.

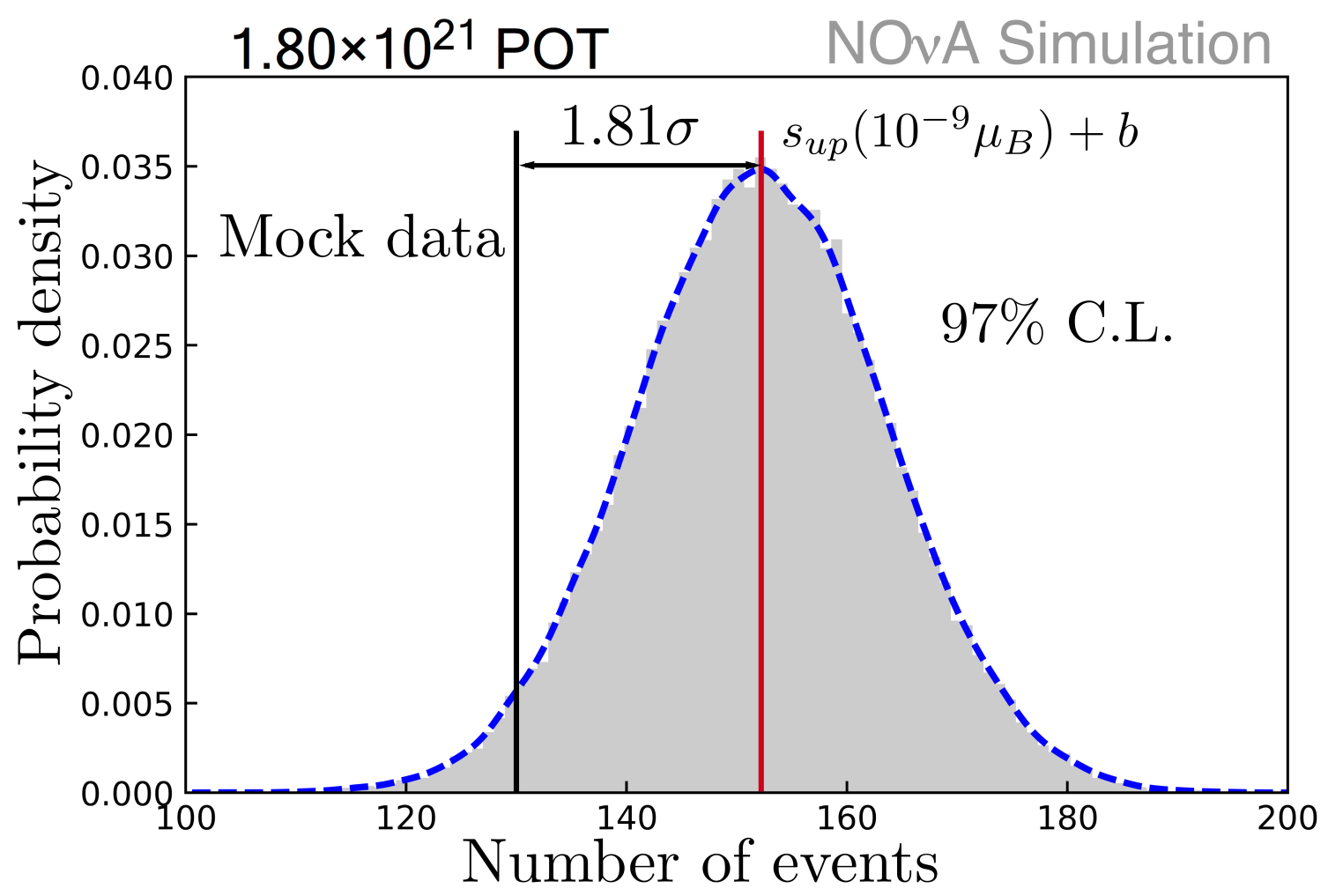

Figure 5.12. The future sensitivity for muon neutrino magnetic moment of $10^{-9} \mu_{B}$ by using $1.8 \times 10^{21}$ POT. The left (black) vertical line is the mock data and the right (red) vertical line is the $s\left(10^{-9} \mu_{B}\right)+b$. The mock data is the same amount of events number as Standard Model prediction. The confidence level for constraining the size of $\mu_{\nu}=10^{-9} \mu_{B}$ for a fixed number of observed events 131, is calculated by using the area on the right hand side of the black line divided by the total area in the distribution.

This means that the value of $10^{-9} \mu_{B}$ is too large for the $90 \%$ C.L. upper limit. Next, we gradually decrease the simulated muon neutrino magnetic moment from $10^{-9} \mu_{B}$, which corresponding to $1.81 \sigma$ as shown in Fig. 5.12, until we match the value of $\mathrm{s}$ that satisfies

$$
s_{u p}=1.28 \sqrt{b} .
$$




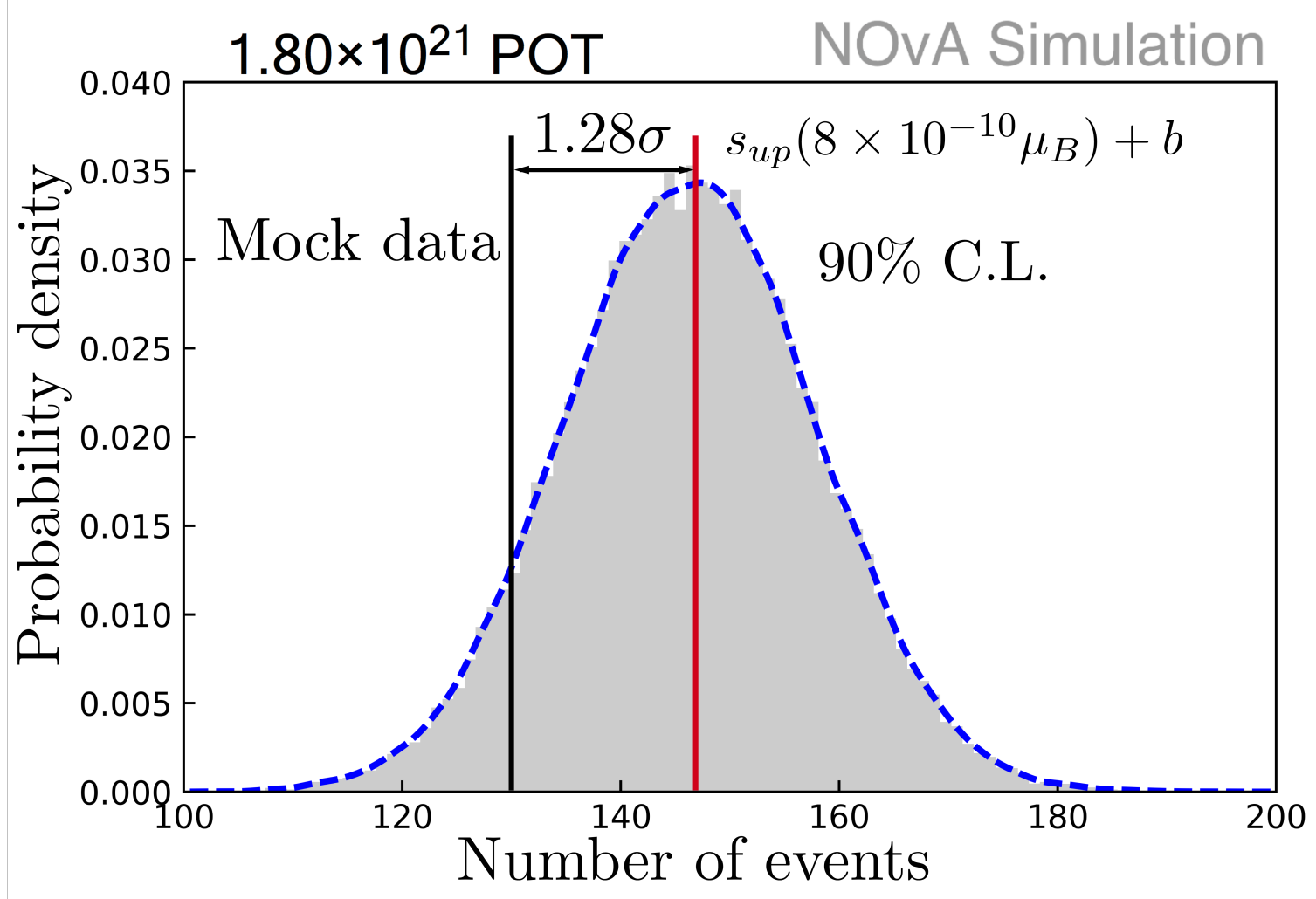

Figure 5.13. The future sensitivity for muon neutrino magnetic moment at 90\% C.L. by using $1.8 \times 10^{21}$ POT. The mock data has the same number of events as the Standard Model predicted. We find $s_{u p}=1.28 \sqrt{b}=14.6$ events, corresponding to $\mu_{\nu}=8 \times 10^{-10} \mu_{B}$.

Then we found that when the value of muon neutrino magnetic moment approaches to $8 \times 10^{-10} \mu_{B}$, the probabilities for number of events no larger than the mock data number adds up to $\alpha=0.1$. Then the sensitivity by using this technique is found (Fig. 5.13).

So that giving the dataset after three years of running at NuMI design intensity in the NOvA near detector, we are able to put an upper limit around $8 \times 10^{-10} \mu_{B}$ without using spectrum fitting under the assumption that the overall number of events in data agree with the Standard Model Monte Carlo prediction. Note that this is the best sensitivity of the muon neutrino magnetic moment in the $\nu_{\mu}$ dominated experiments. 


\section{CHAPTER 6}

\section{Conclusion and Outlook}

In this thesis, we study the $\nu_{\mu}$-e elastic scattering and get good agreement between data and $\mathrm{MC}$ for $1 \mathrm{GeV}<\mathrm{T}<16 \mathrm{GeV}$. By using $3.62 \times 10^{20}$ POT dataset in the NOvA near detector, we find $1.58 \times 10^{-9} \mu_{B}$ as an upper limit (90\% C.L.) for the muon neutrino magnetic moment with $10.9 \%$ systematic uncertainty on the Standard Model background.

We are sensitive to $\mu_{\nu_{\mu}}=8 \times 10^{-10} \mu_{B}$ at the $90 \%$ C.L. upper limit for three years of NuMI designed intensity corresponding to $1.8 \times 10^{21}$ POT. This sensitivity to the muon neutrino magnetic moment is slightly better than previous $\nu_{\mu}$ e dominant experiments in the world. This is under the assumption that the systematic uncertainty on the NOvA Standard Model background is negligible.

The most challenging part of measuring the muon neutrino magnetic moment in accelerator experiments is accurately measuring low energy elastically scattered electrons. Traditional multivariate analyses are very CPU intensive. New deep learning methods from industry such as TenserFlow (Google) and TensorRT (NVIDIA) have the potential to increase the efficiency of the particle identification training steps.

Several charged lepton flavor violation experiments such as mu2e at Fermilab and the COMET experiment in Japan might find this analysis useful, since the muon neutrino to

electron neutrino transitional magnetic moment $\left|\mu_{\mu e}\right|$ scattering could enhance the sensitivity for detecting the $\mu N \rightarrow e N$ channel.

This analysis is motivated by the possibility of a transition magnetic moment of a Majorana muon neutrino. We set an upper limit for $\mu_{\nu_{\mu}}$ and thus examine the triangle inequalities for all Majorana neutrinos [56]. Additionally, violation of these triangular inequalities could hint at the existence of sterile neutrinos [8]. 


\section{BIBLIOGRAPHY}

[1] Adamson, P., Aliaga, L., Ambrose, D., Anfimov, N., et al. Constraints on oscillation parameters from $\nu_{e}$ appearance and $\nu_{\mu}$ disappearance in nova. Phys. Rev. Lett. 118 (Jun 2017), 231801.

[2] Adamson, P., Aliaga, L., Ambrose, D., Anfimov, N., et al. Measurement of the neutrino mixing angle $\theta_{23}$ in nova. Phys. Rev. Lett. 118 (Apr 2017), 151802.

[3] Adamson, P., Aliaga, L., Ambrose, D., Anfimov, N., et al. Search for active-sterile neutrino mixing using neutral-current interactions in nova. Phys. Rev. D 96 (Oct 2017), 072006.

[4] Adamson, P., Anderson, K., Andrews, M., Andrews, R., et Al. The numi neutrino beam. Nuclear Instruments and Methods in Physics Research Section A: Accelerators, Spectrometers, Detectors and Associated Equipment 806 (2016), 279 306.

[5] Adamson, P., et Al. First Measurement of Electron Neutrino Appearance in NOvA. Phys. Rev. Lett. (2016).

[6] Aguilar-Arevalo, A. A., Backfish, M., Bashyal, A., Batell, B., et al. Dark matter search in a proton beam dump with miniboone. Phys. Rev. Lett. 118 (May 2017), 221803.

[7] Ahrens, L. A., Aronson, S. H., Connolly, P. L., Gibbard, B. G., et al. Determination of electroweak parameters from the elastic scattering of muon neutrinos and antineutrinos on electrons. Phys. Rev. D 41 (Jun 1990), 3297-3316.

[8] Alexander, S. Status and perspectives of neutrino magnetic moments. Journal of Physics: Conference Series 718, 6 (2016), 062076.

[9] Aliaga Soplin, L. Neutrino Flux Prediction for the NuMI Beamline. PhD thesis, William-Mary Coll., 2016.

[10] Allen, R. C., Chen, H. H., Doe, P. J., Hausammann, R., et al. Study of electron-neutrino ${ }^{-}$electron elastic scattering at lampf. Phys. Rev. D 47 (Jan 1993), $11-28$.

[11] Allison, J., ET Al. Geant4 developments and applications. IEEE Trans. Nucl. Sci. 53 (2006), 270. 
[12] An, F. P., ET AL. Evolution of the Reactor Antineutrino Flux and Spectrum at Daya Bay. Submitted to: Phys. Rev. Lett. (2017).

[13] Andreopoulos, C., Barry, C., Dytman, S., Gallagher, H., Golan, T., Hatcher, R., Perdue, G., and Yarba, J. The GeniE Neutrino Monte Carlo Generator: Physics and User Manual.

[14] Andreopoulos, C., Et Al. The GENIE Neutrino Monte Carlo Generator. Nucl. Instrum. Meth. A614 (2010), 87-104.

[15] Arrieta Diaz, E. Observation of Muon Neutrino Charged Current Events in an Off-Axis Horn-Focused Neutrino Beam Using the NOvA Prototype Detector. PhD thesis, Michigan State U., 2014.

[16] Ashtari Esfahani, A., ET Al. Determining the neutrino mass with cyclotron radiation emission spectroscopyProject 8. J. Phys. G44, 5 (2017), 054004.

[17] Auerbach, L. B., ET AL. Measurement of electron - neutrino - electron elastic scattering. Phys. Rev. D63 (2001), 112001.

[18] Aurisano, A., Backhouse, C., Hatcher, R., Mayer, N., Musser, J., Patterson, R., Schroeter, R., And Sousa, A. The NOvA simulation chain. J. Phys. Conf. Ser. 664, 7 (2015), 072002.

[19] Ayres, D. S., et Al. The NOvA Technical Design Report.

[20] Babu, K. S., And Mohapatra, R. N. Model for Large Transition Magnetic Moment of the $\nu_{e}$. Phys. Rev. Lett. 63 (1989), 228.

[21] Backhouse, C., And Patterson, R. B. Library Event Matching event classification algorithm for electron neutrino interactions in the NOvA detectors. Nucl. Instrum. Meth. A7r8 (2015), 31-39.

[22] Baird, M., Bian, J., Messier, M., Niner, E., Rocco, D., And Sachdev, K. Event Reconstruction Techniques in NOvA. J. Phys. Conf. Ser. 664, 7 (2015), 072035.

[23] Baird, M., Grover, D., Kasahara, S., and Messier, M. The NOvA DAQ Monitor System. J. Phys. Conf. Ser. 664, 8 (2015), 082020.

[24] BaIrd, M. D. An Analysis of Muon Neutrino Disappearance from the NuMI Beam Using an Optimal Track Fitter. PhD thesis, Indiana U., 2015.

[25] Barut, A. O., And Aydin, Z. Z. ANGUlar DiSTRIBUtion IN ELECTRON-NEUTRINO SCATTERING AND THE ANOMALOUS MAGNETIC MOMENT OF THE NEUTRINO. Nuovo Cim. A101 (1989), 677.

[26] Barut, A. O., Aydin, Z. Z., And Duru, I. H. UPper limit ON THE MAGNETIC MOMENT OF THE NEUTRINO. Phys. Rev. D26 (1982), 1794-1797. 
[27] Bays, K., and Pawloski, G. Status of cross-section tuning. NOvA internal DocDB 24574.

[28] Beda, A. G., Brudanin, V. B., Egorov, V. G., Medvedev, D. V., Pogosov, V. S., Shevchik, E. A., Shirchenko, M. V., Starostin, A. S., And Zhitnikov, I. V. Gemma experiment: The results of neutrino magnetic moment search. Phys. Part. Nucl. Lett. 10 (2013), 139-143.

[29] Beda, A. G., Brudanin, V. B., Egorov, V. G., Medvedev, D. V., Pogosov, V. S., Shirchenko, M. V., and Starostin, A. S. The results of search for the neutrino magnetic moment in GEMMA experiment. Adv. High Energy Phys. 2012 (2012), 350150.

[30] Bell, N. F., Cirigliano, V., Ramsey-Musolf, M. J., Vogel, P., and Wise, M. B. How magnetic is the dirac neutrino? Phys. Rev. Lett. 95 (Oct 2005), 151802.

[31] Bell, N. F., Gorchtein, M., Ramsey-Musolf, M. J., Vogel, P., And WANG, P. Model independent bounds on magnetic moments of Majorana neutrinos. Phys. Lett. B642 (2006), 377-383.

[32] Bezdek, J. C. Pattern recognition with fuzzy objective function algorithms. Springer Science \& Business Media, 2013.

[33] Bian, J. First Results of $\nu_{e}$ Appearance Analysis and Electron Neutrino Identification at NOvA, Proceedings, Meeting of the APS Division of Particles and Fields (DPF 2015): Ann Arbor, Michigan, USA, 4-8 Aug 2015.

[34] Bian, J., ET AL. Pi0 pid for neutrino-electron scattering. NOvA internal DocDB 13334.

[35] Boyarkin, O. M., And Boyarkina, G. G. Electromagnetic properties of neutrinos in the left-right model. Phys. Rev. D90, 2 (2014), 025001.

[36] Bhlen, T., Cerutti, F., Chin, M., Fass, A., Ferrari, A., Ortega, P., Mairani, A., Sala, P., Smirnov, G., and Vlachoudis, V. The fluka code: Developments and challenges for high energy and medical applications. Nuclear Data Sheets 120 (2014), $211-214$.

[37] Campanella, M., Ferrari, A., Sala, P. R., and Vanini, S. First Calorimeter Simulation with the FLUGG Prototype.

[38] Chou, C. N. The nature of the saturation effect of fluorescent scintillators. Phys. Rev. 87 (Sep 1952), 904-905.

[39] Coc, A., And Vangioni, E. Big-bang nucleosynthesis with updated nuclear data. Journal of Physics: Conference Series 202, 1 (2010), 012001.

[40] Coelho, J. A. A. B., Mann, W. A., and Bashar, S. S. Nonmaximal $\theta_{23}$ mixing at nova from neutrino decoherence. Phys. Rev. Lett. 118 (May 2017), 221801. 
[41] Cowan, G. Statistical methods for particle physics. Lectures on Statistics HCPSS Fermilab 11,12 August 2016.

[42] Cowan, G. Statistical Data Analysis. Clarendon Press, 1998. Equation 9.17 on page 127.

[43] Cowan, G., Cranmer, K., Gross, E., and Vitells, O. Asymptotic formulae for likelihood-based tests of new physics. The European Physical Journal C 71, 2 (Feb 2011), 1554.

[44] Cowan, G., Cranmer, K., Gross, E., and Vitells, O. Power-Constrained Limits.

[45] DeCamp, D., Deschizeaux, B., Lees, J.-P., Minard, M.-N., et Al. determination of the number of light neutrino species. Physics Letters B 231, 4 (1989), $519-529$.

[46] Del Tutro, M. Neutrino Beam Simulations and Data Checks for the NOvA Experiment. PhD thesis, Rome U., 2015.

[47] DeNiverville, P., Pospelov, M., And Ritz, A. Observing a light dark matter beam with neutrino experiments. Phys. Rev. D84 (2011), 075020.

[48] Dicus, D. A., Repko, W. W., And Vega, R. Measuring the neutrino mass using intense photon and neutrino beams. Phys. Rev. D 62 (Oct 2000), 093027.

[49] Donath, A., Deil, C., Arribas, M. P., King, J., Owen, E., Terrier, R., Reichardt, I., Harris, J., Bhler, R., And Klepser, S. Gammapy - A Python package for gamma-ray astronomy.

[50] Dorenbosch, J., et Al. EXPERIMENTAL RESULTS ON NEUTRINO ELECTRON SCATTERING. Z. Phys. C41 (1989), 567. [Erratum: Z. Phys.C51,142(1991)].

[51] Duyang, H. Measurement of Neutral Current Coherent $\pi^{0}$ Production In The NOvA Near Detector.

[52] Feldman, G. A rookie's guide to nova's physics and detectors. NOvA summer school, 22 July 2014, NOvA internal DocDB 11664.

[53] Feldman, G. J., And Cousins, R. D. A Unified approach to the classical statistical analysis of small signals. Phys. Rev. D57 (1998), 3873-3889.

[54] Fischler, M., Green, C., And Kowalkowski, J. . A. O. NOvA Event Building, Buffering and Data-Driven Triggering From Within the DAQ System. J. Phys. Conf. Ser. 396 (2012), 012020.

[55] Formaggio, J. A., And Zeller, G. P. From ev to eev: Neutrino cross sections across energy scales. Rev. Mod. Phys. 84 (Sep 2012), 1307-1341. 
[56] Frre, J.-M., Heeck, J., And Mollet, S. Triangle Inequalities for Majorana-Neutrino Magnetic Moments. Phys. Rev. D92, 5 (2015), 053002.

[57] Fukugita, M., and Yanagida, T. A Particle Physics Model for Voloshin-Vysotskii-Okun Solution to the Solar Neutrino Problem. Phys. Rev. Lett. 58 (1987), 1807.

[58] Fuller, G. M., AND Smith, C. J. Nuclear weak interaction rates in primordial nucleosynthesis. Phys. Rev. D 82 (Dec 2010), 125017.

[59] Giunti, C., And Kim, C. W. Fundamentals of Neutrino Physics and Astrophysics. 2007.

[60] Giunti, C., And Studenikin, A. Neutrino electromagnetic interactions: a window to new physics. Rev. Mod. Phys. 87 (2015), 531.

[61] Greiner, W., And Müller, B. Gauge theory of weak interactions. Springer Science \& Business Media, 2009.

[62] Gribov, V. N., And Pontecorvo, B. Neutrino astronomy and lepton charge. Phys. Lett. $28 B$ (1969), 493.

[63] Hagmann, C., Lange, D., And Wright, D. Cosmic-ray shower generator (CRY) for monte carlo transport codes. In Nuclear Science Symposium Conference Record, 200\%. NSS'0\%. IEEE (2007), vol. 2, IEEE, pp. 1143-1146.

[64] Hasert, F. J., Et Al. Search for Elastic $\nu_{\mu}$ Electron Scattering. Phys. Lett. $46 B$ (1973), 121-124.

[65] Kayser, B., Gibrat-Debu, F., and Perrier, F. The Physics of massive neutrinos. World Sci. Lect. Notes Phys. 25 (1989), 1-117.

[66] Kopp, S. E. Accelerator neutrino beams. Physics Reports 439, 3 (2007), 101-159.

[67] Kyuldjiev, A. V. Searching for Effects of Neutrino Magnetic Moments at Reactors and Accelerators. Nucl. Phys. B243 (1984), 387-397.

[68] Lindner, M., Radovi, B., And Welter, J. Revisiting Large Neutrino Magnetic Moments.

[69] Liu, D. Search for non-zero neutrino magnetic moments using Super-Kamiokande-I solar neutrino data. PhD thesis, UC, Irvine, 2005.

[70] LiU, D. W., ET AL. Limits on the neutrino magnetic moment using 1496 days of Super-Kamiokande-I solar neutrino data. Phys. Rev. Lett. 93 (2004), 021802.

[71] Lundberg, J., Conrad, J., Rolke, W., and Lopez, A. Limits, discovery and cut optimization for a Poisson process with uncertainty in background and signal efficiency: TRolke 2.0. Comput. Phys. Commun. 181 (2010), 683-686. 
[72] Marciano, W. J., and Parsa, Z. Neutrino electron scattering theory. J. Phys. G29 (2003), 2629-2645.

[73] Mariani, C., Cheng, G., Conrad, J. M., and Shaevitz, M. H. Improved parametrization of $K^{+}$production in $p$-be collisions at low energy using feynman scaling. Phys. Rev. D 84 (Dec 2011), 114021.

[74] Messier, M. D. First neutrino oscillation measurements in NOvA. Nucl. Phys. B908 (2016), 151-160.

[75] Mufson, S., ET Al. Liquid scintillator production for the NOvA experiment. Nucl. Instrum. Meth. A799 (2015), 1-9.

[76] Niner, E. D. Observation of Electron Neutrino Appearance in the NuMI Beam with the NOvA Experiment. PhD thesis, Indiana U., 2015.

[77] Ning, P., ET AL. Medium and High Energy Probe for Nucleon Inner Structure. 2012.

[78] Ouedraogo, S. A. Limit on the muon neutrino magnetic moment and a measurement of the CCPIP to CCQE cross section ratio. PhD thesis, Louisiana State U., 2008.

[79] Patrignani, C., et Al. Review of Particle Physics. Chin. Phys. C40, 10 (2016), 100001.

[80] Patterson, R. B. The NOvA Experiment: Status and Outlook. [Nucl. Phys. Proc. Suppl.235-236,151(2013)].

[81] Patterson, R. B. Prospects for Measurement of the Neutrino Mass Hierarchy. Ann. Rev. Nucl. Part. Sci. 65 (2015), 177-192.

[82] Pershey, D. Thechnical note for the ccpi02017 analysis. NOvA internal DocDB 18768.

[83] Prosper, H. B. Workshop on Confidence Limits. NATO Sci. Ser. C 566 (2001), 389-399.

[84] Raddatz, N. J. Measurement of Muon Neutrino Disappearance with Non-Fiducial

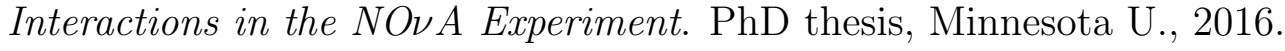

[85] Radovic, A., ET AL. A technote describing the derivation and size of numi flux uncertainties used in the second nova analyses. NOvA internal document 15296v5.

[86] Rodejohann, W., Xu, X.-J., And Yaguna, C. E. Distinguishing between dirac and majorana neutrinos in the presence of general interactions. Journal of High Energy Physics 2017, 5 (2017), 24.

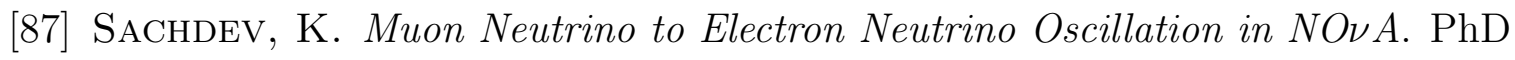
thesis, Minnesota U., 2015. 
[88] Schechtman, G., Schlumprecht, T., and Zinn, J. On the gaussian measure of the intersection. Ann. Probab. 26, 1 (01 1998), 346-357.

[89] Seabold, S., And Perktold, J. Statsmodels: Econometric and statistical modeling with python. In 9th Python in Science Conference (2010).

[90] Sustrand, T., Mrenna, S., And Skands, P. Pythia 6.4 physics and manual. Journal of High Energy Physics 2006, 05 (2006), 026.

[91] Studenikin, A. Neutrino magnetic moment: a window to new physics. Nuclear Physics B - Proceedings Supplements 188 (2009), 220 - 222. Proceedings of the Neutrino Oscillation Workshop.

[92] Talaga, R. L., Grudzinski, J. J., Phan-Budd, S., Pla-Dalmau, A., Fagan, J. E., Grozis, C., And Kephart, K. M. PVC Extrusion Development and Production for the NOvA Neutrino Experiment.

[93] Thomson, M. Future neutrino facilities. SLAC Summer Institute: The Universe of Neutrinos, August 21, 2015.

[94] Vahle, P. New results from NOvA. In The XXVII International Conference on Neutrino Physics and Astrophysics (Neutrino 2016), London (2016).

[95] Vassh, N., Grohs, E., Balantekin, A. B., And Fuller, G. M. Majorana Neutrino Magnetic Moment and Neutrino Decoupling in Big Bang Nucleosynthesis. Phys. Rev. D92, 12 (2015), 125020.

[96] Vega, R., Dicus, D. A., And Repko, W. W. Neutrino-photon scattering and the neutrino mass. 57-63.

[97] Vilain, P., Wilquet, G., Beyer, R., Flegel, W., Et Al. Experimental study of electromagnetic properties of the muon neutrino in neutrino-electron scattering. Physics Letters B 345, 1 (1995), 115 - 118.

[98] Vinton, L. Tech note describing the birks systematics for the second muon neutrino disappearance analysis. NOvA internal DocDB 15347.

[99] Vinton, L. Measurement of Muon Neutrino Disappearance with the NOvA Experiment. PhD thesis, University of Sussex, 2017.

[100] Vogel, P., And Engel, J. Neutrino Electromagnetic Form-Factors. Phys. Rev. D39 (1989), 3378.

[101] Wang, B. Neutrino magnetic moment and lightweight dark matter update. NOvA internal DocDB 15108.

[102] Wang, B., Bian, J., Coan, T. E., Kotelnikov, S., Et Al. Muon neutrino on electron elastic scattering in the nova near detector and its applications beyond the standard model. Journal of Physics: Conference Series 888, 1 (2017), 012123. 
[103] Wilks, S. S. The Large-Sample Distribution of the Likelihood Ratio for Testing Composite Hypotheses. Annals Math. Statist. 9, 1 (1938), 60-62.

[104] Xin, T. Observing Muon Neutrino to Electron Neutrino Oscillations in the NOA Experiment. PhD thesis, Iowa State U., 2016.

[105] Zalesak, J. The nova neutrino experiment. HEP section at the Institute of Physics, CAS in Prague, February 16, 2017. NOvA internal DocDB 17112.

[106] Zhu, Y. Experimental Data Analysis, first ed. Science Press, Beijing, Northern Street on East Side of Imperial Palace Wall for Forbidden City, 2012.

[107] Zirnstein, J. Data driven triggers for the nova experiment. APS DPF2013 Meeting, August 15, 2013. 